\title{
Multidimensional supernova simulations with approximative neutrino transport
}

\section{Neutron star kicks and the anisotropy of neutrino-driven explosions in two spatial dimensions ${ }^{\star}$}

\author{
L. Scheck, K. Kifonidis, H.-Th. Janka, and E. Müller
}

Max-Planck-Institut für Astrophysik, Karl-Schwarzschild-Straße 1, 85741 Garching, Germany
e-mail: scheck@mpa-garching.mpg.de

Received 13 January 2006 / Accepted 12 June 2006

\begin{abstract}
We study hydrodynamic instabilities during the first seconds of core-collapse supernovae by means of 2D simulations with approximative neutrino transport and boundary conditions that parameterize the effects of the contracting neutron star and allow us to obtain sufficiently strong neutrino heating and, hence, neutrino-driven explosions. Confirming more idealised studies, as well as supernova simulations with spectral transport, we find that random seed perturbations can grow by hydrodynamic instabilities to a globally asymmetric mass distribution in the region between the nascent neutron star and the accretion shock, leading to a dominance of dipole $(l=1)$ and quadrupole $(l=2)$ modes in the explosion ejecta, provided the onset of the supernova explosion is sufficiently slower than the growth time scale of the low-mode instability. By gravitational and hydrodynamic forces, the anisotropic mass distribution causes an acceleration of the nascent neutron star, which lasts for several seconds and can propel the neutron star to velocities of more than $1000 \mathrm{~km} \mathrm{~s}^{-1}$. Because the explosion anisotropies develop chaotically and change by small differences in the fluid flow, the magnitude of the kick varies stochastically. No systematic dependence of the average neutron star velocity on the explosion energy or the properties of the considered progenitors is found. Instead, the anisotropy of the mass ejection, and hence of the kick, seems to increase when the nascent neutron star contracts more quickly, and thus low-mode instabilities can grow more rapidly. Our more than 70 models separate into two groups, one with high and the other with low neutron star velocities and accelerations after one second of post-bounce evolution, depending on whether the $l=1$ mode is dominant in the ejecta or not. This leads to a bimodality of the distribution when the neutron star velocities are extrapolated to their terminal values. Establishing a link to the measured distribution of pulsar velocities, however, requires a much larger set of calculations and ultimately 3D modelling.
\end{abstract}

Key words. hydrodynamics - instabilities - radiative transfer - neutrinos - stars: supernovae: general - pulsars: general

\section{Introduction}

Spectropolarimetry (Leonard et al. 2006; Wang et al. 2003, 2001, and references therein) indicates that global anisotropies are a common feature of many core-collapse supernovae $(\mathrm{SNe})$. The deeper one can look into the expanding and increasingly transparent supernova ejecta, the higher is the asymmetry inferred from the observed polarisation. This indicates that the origin of the anisotropy seems to be intrinsically linked to the mechanism of the explosion.

Recent high-resolution imaging of SN 1987 A with the Hubble Space Telescope (Wang et al. 2002), as well as high values for the measured space velocities of young galactic pulsars, may be interpreted as supporting such a link. The average pulsar velocities are as high as $200-500 \mathrm{~km} \mathrm{~s}^{-1}$, and some neutron stars (NSs) move through interstellar space with more than $1000 \mathrm{~km} \mathrm{~s}^{-1}$ (e.g. Cordes et al. 1993; Lyne \& Lorimer 1994; Hansen \& Phinney 1997; Zou et al. 2005; Chatterjee et al. 2005). Claims of a bimodality of the pulsar velocity distribution are still controversial. While some authors have obtained evidence of such a bimodality (Cordes \& Chernoff 1998; Fryer et al. 1998; Arzoumanian et al. 2002; Brisken et al. 2003), others have found

^ Appendices A-D are only available in electronic form at http://www . edpsciences.org that a simple Maxwellian fit works best (Hansen \& Phinney 1997; Hobbs et al. 2005).

Binary disruption, e.g. as a consequence of the SN explosions that give birth to the NSs, does not lead to sufficiently high velocities. Furthermore, the orbital parameters of many binary systems imply an intrinsic acceleration mechanism of the pulsars, probably linked to their creation (see Lai 2001; Lai et al. 2001 for reviews). Quite a number of explanations have been suggested, mostly involving anisotropic mass ejection in the SN explosion or anisotropic neutrino emission of the cooling, nascent NS. The former suggestion might be supported by the fact that some pulsars seem to propagate in a opposite direction to the mass distribution asymmetries of their associated SN remnants. But clear observational evidence is missing, and hydrodynamic simulations have previously either produced rather small recoil velocities (Janka \& Müller 1994), or started from the assumption that a dipolar asymmetry was already present in the pre-collapse iron core giving rise to a large anisotropy of the SN explosion (Burrows \& Hayes 1996). The origin of such big pre-collapse perturbations, however, is not clear (Murphy et al. 2004).

Suggestions that a "neutrino rocket engine" boosts NSs to high velocities (e.g. Chugai 1984; Dorofeev et al. 1985; Burrows \& Woosley 1986; Woosley 1987; for reviews see Lai 2001 
and Lai et al. 2001) make use of the fact that the huge reservoir of gravitational binding energy released during the collapse of the stellar core is mostly carried away by the neutrinos. Creating a global emission anisotropy of these neutrinos of even only $1 \%$ - which is sufficient for obtaining an NS recoil of about $300 \mathrm{~km} \mathrm{~s}^{-1}-$, however, turned out to be very difficult. Most ideas refer to unknown neutrino properties (e.g., Fuller et al. 2003; Fryer \& Kusenko 2006 and refs. therein) and/or require the presence of a very strong magnetic field with a large dipole component (instead of being randomly structured and variable with time) in the newly formed NS (e.g. Arras \& Lai 1999a,b; Socrates et al. 2005). Such assumptions are not accepted generally and are not the result of self-consistent calculations, but are put into the models "by hand".

If the observed high pulsar velocities indeed go back to the early moments of the SN explosion, the simplest explanation would certainly be a common origin for explosion asymmetries and pulsar acceleration. In this case anisotropic ejection of mass would lead to a recoil (or "kick") of the NS due to (linear) momentum conservation. Various kinds of hydrodynamic instabilities might in fact be responsible for a large-scale deformation of the ejecta and globally aspherical explosions. Perturbation analysis of volume-filling thermal convection in a fluid sphere by Chandrasekhar (1961) found the highest growth rates for the $l=1, m=0$ mode (in terms of an expansion in spherical harmonics $Y_{l}^{m}$ of order $l, m$ ). This is supported by (full $4 \pi$ ) 3D simulations of convection in red giant and non-rotating main sequence stars (Woodward et al. 2003; Kuhlen et al. 2003). Motivated by Chandrasekhar's analysis, Herant (1995) speculated about the formation of a stable $l=1, m=0$ convective mode in the neutrino-heated layers between the gain radius and the supernova shock. In this configuration only a single buoyant bubble (outflow) exists, along with a single accretion funnel (inflow), which reaches from the postshock region down to the NS. Herant (1995) suggests the potential importance of such a convective pattern for NS kicks up to nearly $1000 \mathrm{~km} \mathrm{~s}^{-1}$. Instability of the accretion shock to a global Rayleigh-Taylor mode that could lead to asymmetric shock expansion and a net recoil of the NS of several $100 \mathrm{~km} \mathrm{~s}^{-1}$ has also been predicted by Thompson (2000). However, according to the linear analysis by Foglizzo et al. (2006), advection tends to stabilise the growth of longwavelength perturbations in the neutrino-heated accretion flow behind the standing shock. A convective trigger of such instabilities therefore requires the local growth rate to exceed a critical threshold value.

Non-radial, low-mode instability of shocked accretion flows can also be caused by the "advective-acoustic cycle". In the astrophysical context, this instability was first discussed by Foglizzo \& Tagger (2000) and Foglizzo (2001, 2002) in an application to the Bondi-Hoyle accretion of black holes. It was more recently considered for supernova-core-like conditions by Galletti \& Foglizzo (2005). This instability relies on the fact that the infall of entropy and vorticity perturbations produces acoustic waves that propagate outward and create new entropy and vorticity perturbations when reaching the shock, thereby closing an amplifying feedback cycle that eventually results in a dominant $l=1$ or $l=2$ mode. The advective-acoustic cycle can even operate under conditions in which convective instabilities are hampered, e.g. if the advection of matter out of the convectively unstable region is too fast to allow for a significant convective growth of small perturbations.

By means of numerical simulations, Blondin et al. (2003) investigated an idealised setup for the stalled shock in a supernova core and showed that a spherical shock is dynamically unstable to non-radial perturbations, even without neutrino heating and convection. The authors referred to this as the "standing accretion shock instability", or SASI, which reveals a preferred growth of $l=1$ mode deformation and which was explained by Blondin \& Mezzacappa (2006) as a consequence of the propagation of sound waves in the volume enclosed by the shock.

While these investigations lacked a detailed description of the neutrino physics and of the equation of state of the supernova medium, Scheck et al. (2004); Janka et al. (2005b, 2004b, 2005a), Ohnishi et al. (2006), Buras et al. (2006b), and Burrows et al. (2006) provide results that demonstrate that the instability of the accretion shock also occurs in models that include the relevant microphysics with more realism. Scheck et al. (2004) suggested a link of these low-mode instabilities of the supernova shock during the neutrino-heating phase to global explosion asymmetries (see in particular Kifonidis et al. 2006) and pulsar kicks. Most previous 2D simulations of successful neutrino-driven explosions (Herant et al. 1992; Herant et al. 1994; Burrows \& Fryxell 1993; Burrows et al. 1995) have failed to see the development of $l=1,2$ modes (such an anisotropy, however, showed up in one of the weakly exploding models of Janka \& Müller 1996), because most of the simulations were done with limited computational wedges of only $90^{\circ}$ to $120^{\circ}$ latitudinal width or because very rapid explosions were obtained. In these cases the low-mode asymmetries were excluded by constraining boundary conditions, or they could not grow in the time available between shock stagnation and revival. This effect may have been the reason why low-mode instabilities were not found to be dominant in the 3D simulations of Fryer \& Warren (2002, 2004), which developed explosions on rather short time scales after bounce. It is also possible that these 3D simulations were not evolved far enough in time to observe the formation of $l=1,2$ modes. Without a sufficiently strong contribution of the $l=1$ mode, the neutron star recoil velocities remain low (typically less than about $200 \mathrm{~km} \mathrm{~s}^{-1}$, see Janka \& Müller 1994).

The main goal of the present paper (the first in a series) is to show that global anisotropies and large NS kicks can be obtained naturally in the framework of the neutrino-driven SN explosion mechanism due to the symmetry breaking by non-radial hydrodynamic instabilities, without the need to resort to rapid rotation (e.g. Kotake et al. 2003), large pre-collapse perturbations in the iron core (Burrows \& Hayes 1996; Goldreich et al. 1996; Lai \& Goldreich 2000), strong magnetic fields (Wheeler et al. 2002; Kotake et al. 2004), anisotropic neutrino emission associated with exotic neutrino properties (e.g. Fryer \& Kusenko 2006; Fuller et al. 2003), or jets (Cen 1998; Khokhlov et al. 1999; Lai et al. 2001). To this end we present a comprehensive 2D parameter study of supernova dynamics that can be considered as a significant improvement and extension of the earlier calculations of Janka \& Müller (1996) with respect to the treatment of neutrino transport, the assumed characteristics of the neutrino emission from the neutron star core, the inclusion of rotation, the influence of the initial seed perturbations, the spatial resolution, and the covered evolutionary time of the supernova explosions.

Parts of the present work already have been presented in a Letter by Scheck et al. (2004), but a detailed description of both our methods and results will be given here. We proceed by summarising our numerical algorithms and computational approach in Sect. 2, and our boundary conditions and initial data in Sect. 3. We then give an overview of our simulations in Sect. 4, discussing two representative neutrino-hydrodynamic calculations in some detail. In Sect. 5 we explore the dependence of our simulations on the properties of the stellar progenitors and on the assumed core neutrino fluxes, and establish correlations 
between explosion parameters and neutron star kicks. Section 6 is devoted to the effects of rotation. In Sect. 7 we return to the neutron star recoils and investigate their robustness with respect to the approximations and assumptions that we have employed. Furthermore, we investigate the long-time evolution of the recoil velocities for a few models beyond the time interval of one second after core bounce, for which we have evolved most of our models. Estimating the terminal values of the NS velocities by a calibrated extrapolation procedure, we speculate about the possible implications of our results for the velocity distribution of neutron stars in Sect. 8. A summary of this work and our conclusions can be found in Sect. 9. In Appendix A we define and tabulate some physical quantities of interest that characterise the different runs of our large set of simulations. Furthermore, we describe the post-processing procedures that we applied to the numerical calculations to compute these characteristic quantities. Appendix B discusses the solution of the hydrodynamics equations in an accelerated frame of reference. In Appendix C we analyse the explosion energetics of our neutrino-driven supernovae. Appendix D finally explains our new neutrino transport scheme in detail.

\section{Computational approach and numerical methods}

In this section the employed numerical codes for hydrodynamics and neutrino transport will be briefly described.

\subsection{Hydrodynamics and gravity}

The basic version of the computer program that we employed for this study is described in Kifonidis et al. (2003). It consists of a hydrodynamics module, which is based on the direct Eulerian version of the piecewise parabolic method (PPM) of Colella \& Woodward (1984) (augmented by the HLLE solver of Einfeldt 1988 to avoid the odd-even-decoupling instability), and on a module that computes the source terms for energy and lepton number that enter the hydrodynamic equations due to neutrino absorption, scattering, and emission processes (see below). The equation of state is that of Janka \& Müller (1996). In contrast to Kifonidis et al. (2003, 2006), we do not follow explosive nucleosynthesis in this work. This allows us to save a considerable amount of computer time, which is mandatory for carrying out an extended parameter study like the one presented here. For the same reason, the neutrino transport in our simulations is described approximately (see Sect. 2.2) and the dense NS core is replaced by a moving inner boundary (usually a Lagrangian shell) whose contraction mimics the shrinking proto-neutron star.

We include self-gravity with relativistic corrections by first solving the Newtonian 2D Poisson equation using a Legendre expansion according to Müller \& Steinmetz (1995), and by subsequently replacing the "spherical part" of the resulting gravitational potential of the 2D mass distribution by the "effective relativistic potential" of Rampp \& Janka (2002) (for details, see Marek et al. 2006). For describing the gravity of the central "point mass" (i.e., the mass enclosed by our inner boundary), we use the baryonic mass where Eq. (53) in Rampp \& Janka (2002) requires the gravitational mass. In our calculations, his turned out to yield very good agreement with the improved version of the effective relativistic potential developed by Marek et al. (2006).

\subsection{Neutrino transport and neutrino source terms}

The original code version of Kifonidis et al. (2003) made use of a simple light-bulb approximation (Janka \& Müller 1996) in which luminosities of neutrinos and antineutrinos of all flavours were imposed at the inner boundary (which is usually below the neutrinospheres) and kept constant with radius. These luminosities were typically not chosen to give accurate values for the fluxes prevailing below the neutrinospheric layers, but their choice was guided by the asymptotic luminosities that emerge from the contracting and accreting nascent neutron star at large radii. This was necessary in order to cope with the main problem of a light-bulb approach, namely that it neglects the changes in the neutrino fluxes and spectra that result from the interactions of neutrinos with the stellar matter, thus ignoring, for example, the contributions of the neutrino emission from accreted matter to the neutrino luminosity.

In this work we considerably improve upon this former approach by explicitly including these effects. We achieve this by abandoning the light bulb in favour of a gray, characteristicsbased scheme that can approximate neutrino transport in the transparent and semi-transparent regimes. The approach is not particularly suited to also handling the regime of very large optical depths, $\tau$. Therefore we still perform our simulations with an inner grid boundary at $\tau \approx 10 \ldots 100$. However, the luminosities prescribed there have no relation to those used in the older light-bulb calculations. We have chosen them to qualitatively reproduce the evolution of the luminosities in a Lagrangian mass shell below the neutrinospheres as obtained in recent Boltzmann transport calculations (see also Sect. 3.2 and especially Appendix D.2 for details).

The transport scheme itself solves the zeroth-order moment equation of the Boltzmann equation. The transport of neutrino number and energy is accounted for separately by integrating two such moment equations for neutrinos and antineutrinos of all flavours $(\mathrm{e}, \mu, \tau)$. This allows us to adopt a non-equilibrium description with the assumption that the spectral form is FermiDirac, but the neutrino temperatures $T_{v_{i}}$ are not necessarily equal to the gas temperature $T$. Solving transport equations for neutrino number and energy, we can locally determine neutrino number and energy densities and thus the spectral temperatures $T_{v_{i}}$ from the mean neutrino energies. A detailed description of our approximative solution of the non-equilibrium transport problem and the exact expressions for the employed interaction kernels can be found in Appendix D. While giving qualitatively similar results as Boltzmann-solvers in spherical symmetry (cf. Sect. 4.3), the computational cost of this approximative transport scheme is two orders of magnitude lower.

\subsection{Numerical grid and frame of reference}

We adopted 2D spherical coordinates $(r, \theta)$ and assumed axisymmetry. Unless noted otherwise, the calculations presented in the following are carried out in the full sphere, i.e. for $0 \leq \theta \leq \pi$, with a grid that is equidistant in the lateral direction. A nonequidistant grid is employed in the radial direction whose local spacing, $\Delta r$, is chosen such that square-shaped cells are obtained in the convective region, i.e. $\Delta r \approx r \Delta \theta$. Typically 400 radial and 180 lateral zones are used.

The outer boundary of the computational domain is typically located at $R_{\mathrm{ob}} \approx 2 \times 10^{4} \mathrm{~km}$, while the inner boundary is placed within the forming neutron star after core bounce, at a Lagrangian mass shell somewhat below the electron neutrinosphere. The spacing of the zones near and below the 
neutrinospheres is chosen such that variations in the optical depth per zone remain smaller than a few. The baryonic matter of the neutron star interior to the inner boundary, $M_{\text {core }}$ (which is typically $\sim 1.1 M_{\odot}$ ), is removed and its gravitational attraction is taken into account by assuming a point mass at $r=0$ (see Sect. 2.1).

Since we are mainly interested in neutron star kicks in this paper, we need to point out that the use of the inner boundary condition (enclosing the NS "core") implies that the NS is attached to the centre of our computational grid. It is therefore not free to move relative to the ejecta during the simulation (unless special measures are taken, see below). This is tantamount to assuming that the NS has an infinite inertial mass. Two implications result from this approximation: a potential hydrodynamic feedback of a displacement of the NS relative to the ejecta is neglected, and the neutron star recoil velocity has to be determined indirectly in a post-processing step by making use of the assumption of total momentum conservation (see Appendix A).

The relative motion between neutron star and ejecta can, however, be accounted for during a simulation by "wagging the dog", i.e. by assuming that instead of the neutron star the ejecta move coherently in the opposite direction of the neutron star's recoil. This can be achieved technically by adding the velocity of the relative motion to the gas velocity on the computational grid, which is tantamount to performing (after every time step) a Galilei transformation to a new inertial frame in which the neutron star core is at rest and centred at $r=0$ (see Appendix B for details). Simulations including this procedure will be used to investigate potential deficiencies of our standard assumption that the NS has an infinite inertial mass and takes up momentum without starting to move (see Sects. 4.4 and 7).

\section{Initial and boundary conditions}

In this section the initial and boundary conditions used for our calculations will be defined.

\subsection{Initial models and initial perturbations}

Our calculations are started at $\sim 15-20 \mathrm{~ms}$ after core bounce from detailed post-collapse models. We make use of four such models that are based on three different SN progenitors. The first was calculated by Bruenn (1993) with a general relativistic, one-dimensional (1D), Lagrangian hydrodynamics code coupled to neutrino transport by multi-group, flux-limited diffusion (see his model WPE15 LS 180). It employs the $15 M_{\odot}$ progenitor of Woosley et al. (1988). Simulations based on this model will henceforth be called the "B-series".

Our second 1D post-collapse model, provided by M. Rampp (priv. comm.), uses a $15 M_{\odot}$ progenitor star of Limongi et al. (2000) and was computed with the PROMETHEUS PPM hydrodynamics code coupled to the VERTEX multi-group variable Eddington factor/Boltzmann neutrino transport solver (Rampp \& Janka 2002). Our "L-series" of simulations makes use of that model.

We also consider two post-bounce models that were computed for the s15s7b2 progenitor of Woosley \& Weaver (1995) with PROMETHEUS/VERTEX by Buras et al. (2003, 2006a) (see their models s15/1D and s15r). The first of these models is from a 1D simulation and gives rise to the "W-series" of runs, while the second is a rotating, 2D (axisymmetric) model, which we use for our "R-series" of calculations. This model is described in detail in Müller et al. (2004).

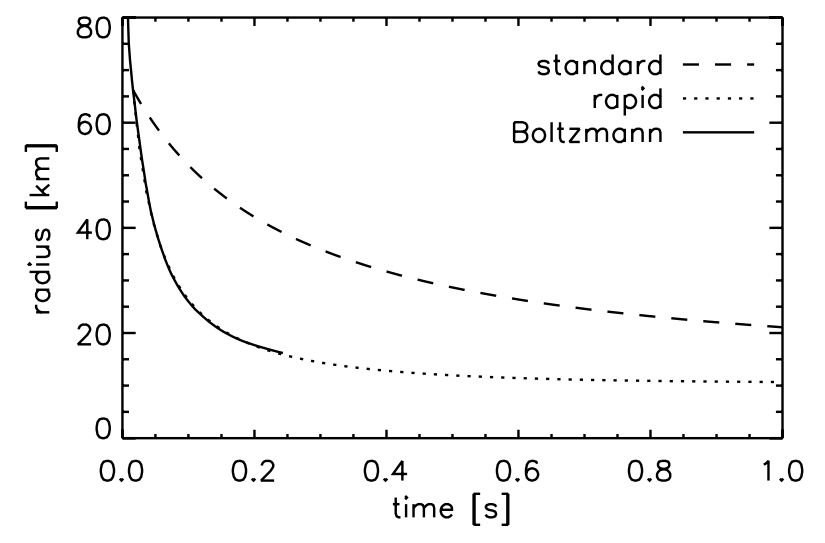

Fig. 1. Evolution after core bounce of the radius corresponding to a mass coordinate of $1.1 M_{\odot}$ from a supernova simulation with Boltzmann neutrino transport (Buras et al. 2003), compared to the motion of the inner boundary radius as defined by Eq. (1) for the standard boundary contraction case (with $t_{L}=1 \mathrm{~s}$ ) and the rapid boundary contraction case.

The level of numerical noise in our hydrodynamics code is so low that a $1 \mathrm{D}$, isotropic initial configuration remains isotropic, even in the presence of a convectively unstable stratification. Therefore we need to explicitly add random perturbations to trigger the growth of non-radial hydrodynamic instabilities in the post-shock flow. The portable, high-quality random number generator RANLUX of James $(1994,1996)$ and Lüscher (1994) is employed. We apply the perturbation to the velocity field and typically use an amplitude of $0.1 \%$. To break the equatorial symmetry of the rotating 2D model of Buras et al. (2003, 2006a), we have to add perturbations with an amplitude of several per cent, since in this model the initial perturbations have already grown to such a level by the time we map the model to our full $180^{\circ}$ grid (see Sect. 2.3).

\subsection{Boundary conditions}

For solving the hydrodynamics equations, reflecting boundary conditions are imposed at the lateral boundaries at $\theta=0$ and $\theta=\pi$, while transmitting (i.e. zero gradient) boundary conditions are employed at the outer radial boundary. The inner boundary, which is located at the Lagrangian mass coordinate where we cut our initial (i.e. immediate post-bounce) models, is taken to be impenetrable. The contraction of this mass shell (and hence of the neutron star core) is mimicked by moving the inner boundary of our Eulerian grid from its initial radius, $R_{\mathrm{ib}}^{\mathrm{i}}$, inwards to a final radius $R_{\mathrm{ib}}^{\mathrm{f}}$ according to the expression

$R_{\mathrm{ib}}(t)=\frac{R_{\mathrm{ib}}^{\mathrm{i}}}{1+\left(1-\exp \left(-t / t_{\mathrm{ib}}\right)\right)\left(R_{\mathrm{ib}}^{\mathrm{i}} / R_{\mathrm{ib}}^{\mathrm{f}}-1\right)}$

of Janka \& Müller (1996). The parameter $R_{\mathrm{ib}}^{\mathrm{i}}$ is typically in the range $55 \mathrm{~km}<R_{\mathrm{ib}}^{\mathrm{i}}<85 \mathrm{~km}$.

For $R_{\mathrm{ib}}^{\mathrm{f}}$ and $t_{\mathrm{ib}}$ we use two alternative prescriptions. In what we henceforth will call the "standard boundary contraction case" - as this is the original parametrization that was employed by Janka \& Müller (1996) - we set $R_{\mathrm{ib}}^{\mathrm{f}}=15 \mathrm{~km}$ and $t_{\mathrm{ib}}=t_{L}$, where the time scale $t_{L}$ is connected to the luminosity decay and is defined in Appendix D.2. In the second prescription, the so-called "rapid boundary contraction case", we set $R_{\mathrm{ib}}^{\mathrm{f}}=10.5 \mathrm{~km}$ and $t_{\mathrm{ib}}=0.25 \mathrm{~s}$.

Figure 1 compares $R_{\mathrm{ib}}(t)$ for both parameter choices with each other and with data from a supernova simulation with the 
nuclear equation of state of Lattimer \& Swesty (1991) and with Boltzmann neutrino transport (Buras et al. 2003) for one of our initial models. The standard boundary contraction leads to a larger final radius and a slower contraction of the neutron star. The rapid boundary contraction gives results that are almost indistinguishable from the Boltzmann calculation. Although the latter parametrization is potentially more realistic than the former, we have performed the simulations with the "standard" case unless noted otherwise. On the one hand, this reduces the differences compared to our previous work to only the treatment of the neutrino transport. On the other hand, the more compact neutron stars obtained with the "rapid" case require the use of a much finer zoning for adequately resolving the steep density drop in the neutron star "atmosphere". This leads to computing times that are at least a factor of five larger than those of the standard case. Exclusive use of the rapidly contracting boundary would thus have severely reduced the number of computed models, leading to much poorer statistics. For this reason we have chosen the rapidly contracting boundary only for a limited set of models to investigate whether the results obtained for the standard case change qualitatively. Both cases together therefore provide information how the results depend on the contraction behaviour of the forming neutron star (see Sect. 7.3).

The boundary conditions for the neutrino emission at the inner grid boundary are chosen to be isotropic. Luminosities and mean energies for neutrinos and antineutrinos of all three flavours are imposed there in order to solve the transport problem as described in Appendix D. These luminosities and energies are chosen as time-dependent functions that are constrained by prescribed and varied values for the total loss of energy and lepton number from the core of the forming neutron star. For example, the lepton number loss during the first second is of the order of $0.1-0.2$ in all our simulations, and the total (asymptotic) energy loss $\Delta E_{v, \text { core }}^{\infty}$ does not exceed the gravitational energy

$E \approx 3 \times 10^{53}\left(\frac{M_{\mathrm{ns}}}{M_{\odot}}\right)^{2}\left(\frac{R_{\mathrm{ns}}}{10 \mathrm{~km}}\right)^{-1} \mathrm{erg} ;$

which can be released during the birth of a neutron star (see Tables A.1-A.5).

\section{Overview of the simulations}

In the following we will give an overview of the simulations based on the "standard" boundary contraction prescription. In particular, we will discuss the character of the flow, the impact of our neutrino treatment, and the acceleration of the neutron star for two exemplary cases.

\subsection{The computed models}

Tables A.1-A.5 give an overview of all our simulations (which were performed with the "standard boundary contraction") in terms of some characteristic quantities that are defined in Appendix A.

The naming convention we have chosen for the models is the following: The first letter denotes the initial model (i.e. the progenitor/post-bounce data), followed by a two-digit code that corresponds to the chosen value for the total asymptotic neutrino energy loss of the neutron star core, $\Delta E_{v, \text { core }}^{\infty}$, in units of $\frac{1}{100} M_{\odot} c^{2}$. Thus B18, for example, refers to a simulation based on the Woosley et al. (1988)/Bruenn (1993) initial data with an assumed release of gravitational binding energy of the core of
$\Delta E_{v, \text { core }}^{\infty}=0.18 M_{\odot} c^{2}$. The second fundamental model parameter, the luminosity time scale $t_{L}$, is not taken into account in the model names, because it has the same value for all models of a series. The chosen value in each case is given in the captions of Tables A.1-A.5.

Simulations performed on a larger grid (with an outer boundary radius of $10^{10} \mathrm{~cm}$ and 500 radial zones) are indicated by the letter " $g$ " appended to the model name, e.g. B18-g, simulations that account for the recoil motion of the neutron star contain the letter " $\mathrm{m}$ " in the model name, and model series started from different random seed perturbations are denoted by numbers appended to the model names. Hence model B18-1 differs from model B18 (and from models B18-2, B18-3 etc.) only in the random perturbations imposed on the initial velocity distribution (with the perturbation amplitude being the same in all cases).

Note that in Tables A.1-A.5 the total lepton number and energy loss of the neutron star core, $\Delta Y_{\mathrm{e}, \text { core }}$ and $\Delta E_{v, \mathrm{tore}}^{\mathrm{tot}}$, respectively, the time-integrated energy loss in $v_{\mathrm{e}}$ and $\bar{v}_{\mathrm{e}}, \Delta E_{500}$, the explosion energy, $E_{\text {exp }}$, the anisotropy parameter, $\alpha_{\text {gas }}$, the shock deformation, $d_{\text {shock}}$, the neutron star mass and recoil velocity, $M_{\mathrm{ns}}$, and $v_{z}^{\mathrm{ns}}$, respectively, as well as the correction of the latter due to the "neutrino rocket effect", $v_{z}^{\text {ns, } v}$, are all given at the time $t=1 \mathrm{~s}$, at which we usually stop our simulations.

We need to point out here that the listed neutron star velocities are not the final ones, but that even at the end of our simulations the neutron stars can still experience a large acceleration. We therefore also give this acceleration, $a_{z}^{\mathrm{ns}}=\mathrm{d} v_{z}^{\mathrm{ns}} / \mathrm{d} t$ (averaged over the last $100 \mathrm{~ms}$ and without neutrino effects), and will attempt to estimate the final neutron star velocities in Sect. 7.4.

\subsection{The character of the flow}

Giving an accurate qualitative description of the flow that establishes in our calculations is a difficult endeavour, as the evolution that we observe during the first $\sim 300-400 \mathrm{~ms}$ is wildly timedependent and extremely nonlinear. One may even characterise it as chaotic. The layer between the proto-neutron star and the supernova shock is Ledoux-unstable, because a negative entropy gradient is established due to neutrino heating within $\sim 50 \mathrm{~ms}$ after bounce. In all simulations discussed here, it is consequently Ledoux convection that breaks the initial spherical symmetry: small Rayleigh-Taylor mushrooms grow from the imposed random seed perturbations and start rising towards the shock. They merge quickly and grow to fewer but larger bubbles that deform the shock and push it outward (Fig. 2).

Due to the violent motions of the rising high-entropy plumes the shock gets bumpy and deformed, and caustic-like kinks of the shock emerge where two such bubbles approach each other and collide. Downstream of the shock, decelerated and compressed matter forms a high-density (low-entropy) shell, which sits atop high-entropy material that boils vigorously as it is heated by neutrinos from below. The interface between these layers is Rayleigh-Taylor unstable (Herant 1995) and gives therefore rise to narrow, low-entropy downflows of matter, which penetrate from the postshock layer to the neutron star with supersonic velocities. When they reach surroundings with entropies lower than their own, the downflows are decelerated and their material spreads rapidly around the neutron star. The evolution of these downflows is highly dynamic. They form, merge with other accretion funnels, or are blown away by the rising buoyant matter on a time scale of $10-20 \mathrm{~ms}$, while their number decreases with time. The most massive of these downflows originate from the kinks at the shock surface, where the deceleration 

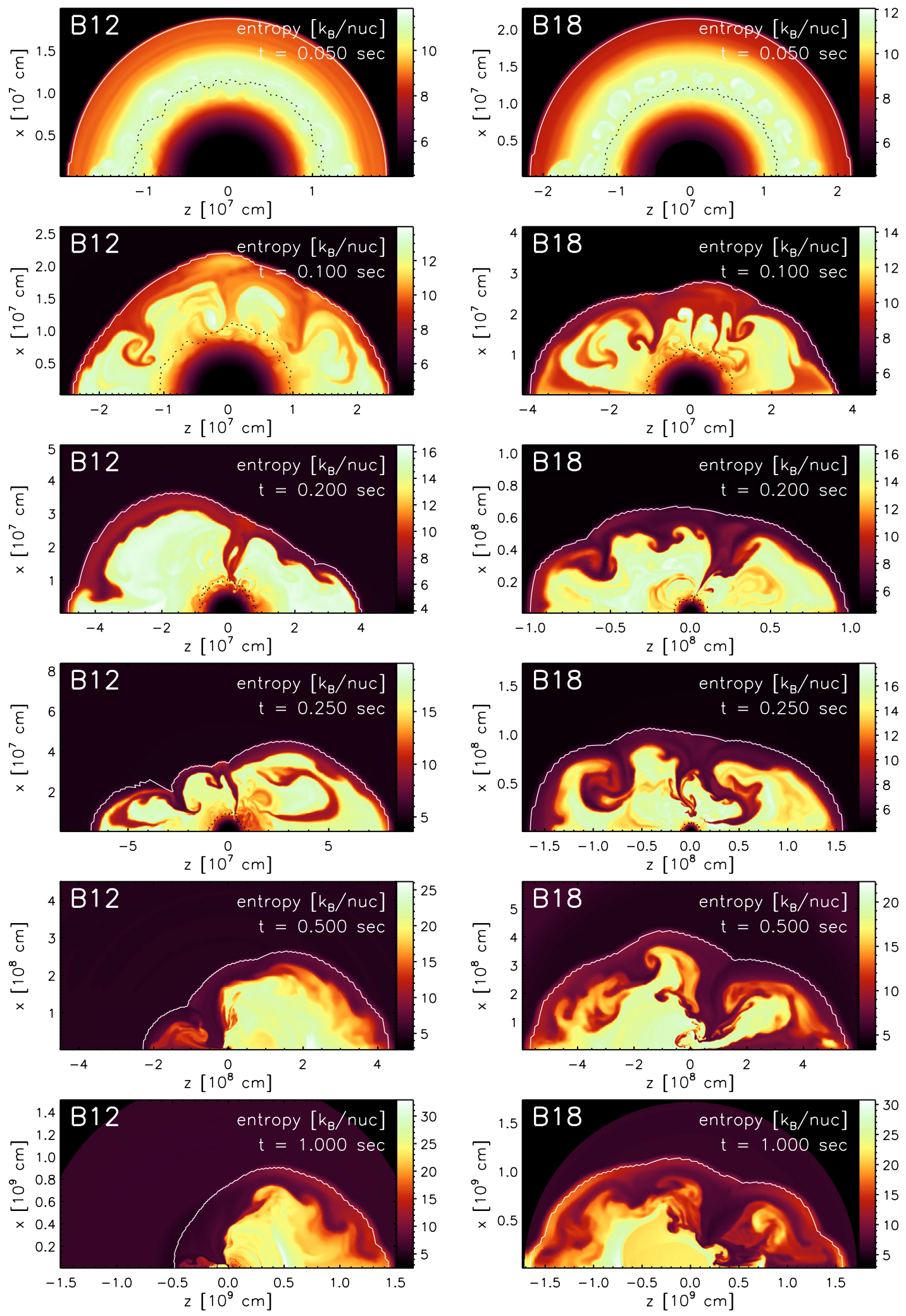

Fig. 2. Entropy distributions in model B12 (left column) and model B18 (right column) for different times. The figures are plotted such that the polar axis is oriented horizontally with "south" $(\theta=\pi)$ on the left and "north" $(\theta=0)$ on the right. Dotted black lines mark the gain radius and white lines the supernova shock. Note that the scales differ between the plots. Convective activity starts with small Rayleigh-Taylor structures $(t=50 \mathrm{~ms})$, which then grow and merge to larger cells and global anisotropy. In contrast to model B18, the low-energy model B12 develops pronounced bipolar oscillations (compare the plots for $t=200 \mathrm{~ms}$ and $t=250 \mathrm{~ms}$ between both cases). After the explosion has set in, the convective pattern "freezes out" and the expansion continues essentially self-similarly (see the plots for $t=500 \mathrm{~ms}$ and $t=1000 \mathrm{~ms}$ ). At $1 \mathrm{~s}$ after bounce model B18 shows the emergence of an essentially spherical neutrino-driven wind expanding away from the neutron star surface (region around the coordinate center in the bottom right panel). 

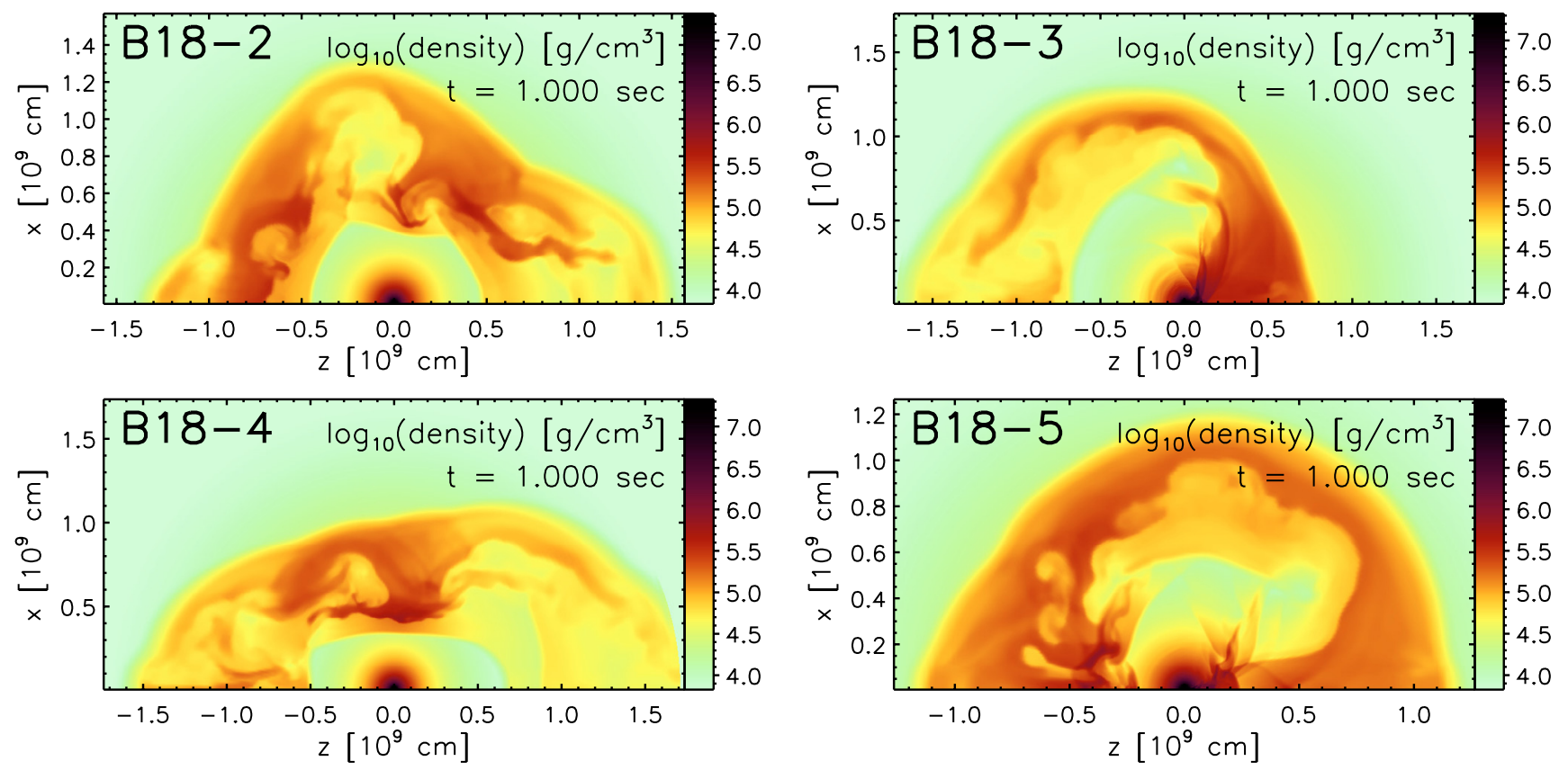

Fig. 3. Density distributions one second after core bounce for four simulations with the same initial and boundary conditions as model B18, but different patterns of the random seed perturbations imposed on the velocity field of the initial model. The amplitudes of the perturbations $\left(10^{-3}\right)$ are the same in all cases. The morphology of the explosion depends in a chaotic way on the initial perturbations.

of the infalling matter is weaker due to the (local) obliqueness of the shock.

During this phase of violent "boiling" the shock develops a strong, time-dependent deformation and expands slowly outward. In model B12, whose evolution is shown in the sequence of plots on the left side of Fig. 2, pronounced bipolar hemispheric oscillations become visible after about $150 \mathrm{~ms}$. Such bipolar oscillations (and the consequent "sloshing") of the shock have been found to be typical of $l=1$ mode instabilities as associated with the advective-acoustic instability or the SASI. Hence these instabilities are likely to dominate the evolution of this model in this strongly nonlinear phase. Note that model B12 differs from model B18 (on the right side of Fig. 2) by lower neutrino luminosities at the inner boundary and, correspondingly, by a later onset of the explosion (at $t_{\exp }=220 \mathrm{~ms}$ compared to $t_{\exp }=152 \mathrm{~ms}$ ) and a lower explosion energy of $0.37 \times 10^{51} \mathrm{erg}$ versus $1.16 \times 10^{51} \mathrm{erg}$ (measured at $t=1 \mathrm{~s}$ ). Model B18 shows also violent convective activity, but no bipolar oscillations. This fact might indicate that in this model the convective instability might play a more important role. A detailed investigation of the growth of different kinds of non-radial instabilities in the postshock flow and their competition will be presented in a subsequent paper of this series (Scheck et al. 2006, in preparation). Here we only note that their combined effects can have a decisive impact on the explosion mechanism of supernovae, since 1D counterparts of both models B12 and B18 failed to explode.

The highly dynamic phase of the evolution comes to an end around $300-400 \mathrm{~ms}$ after bounce. At that time the explosion is well underway, and the overall flow settles into a state of quasiself-similar expansion, which is remarkably stable (compare the lower panels of Fig. 2, and see also Herant 1995). Yet the consequences of the dynamic phase are felt long thereafter, since the strongly nonlinear boiling motions lead to a final morphology that is sensitive to even tiny initial differences in the flow. These may not only result from the influence of different boundary conditions, as in case of models B12 and B18. The late-time morphology is even sensitive to the seed perturbation that we apply to trigger the non-radial instabilities. Figure 3 illustrates this for the B18-series of models in which the seed perturbation was varied as described in Sect. 4.1. It is obvious that the dominant mode in the flow is unpredictable. It can be $l=2$, with two bubbles that are separated by a single accretion funnel, and that occupy roughly a hemisphere each (as in model B18-4 and the original model B18). Yet the bubbles may also differ significantly in size resulting in a dominance of the $l=1$ anisotropy as in model B18-3 (top right panel of Fig. 3) and model B12 (left panels of Fig. 2). This sensitivity to the seed perturbation is so extreme that the system may be described as exhibiting symmetry breaking in a chaotic manner. In fact even the same model computed on different machines (with slightly different 64-bit round off behaviour) may actually end up with a different morphology.

For sufficiently high core luminosities, accretion of matter onto the neutron star is eventually superseded by the onset of a nearly spherically symmetric neutrino-driven wind (see the region around the coordinate center in the lowermost right panel of Fig. 2 and in the left panels of Fig. 3; cf. also Burrows et al. 1995; Janka \& Müller 1996). If the wind is strong enough, as in model B18 where the mass-loss rate of the nascent neutron star by the wind is $\dot{M}_{\mathrm{ns}}=-5.1 \times 10^{-2} M_{\odot} / \mathrm{s}$, it blows away the accretion funnel and establishes a high-entropy shell or cavity of rapidly expanding low-density material around the neutron star, which is separated from the ejecta by a strong reverse shock. Otherwise accretion through the funnel continues until more than about $1 \mathrm{~s}$ after bounce, as in model B12. In this case the accreted material reaches infall velocities of about $1 / 4$ of the speed of light, while the accretion rate at $t=1 \mathrm{~s}$ has decreased to $\dot{M}_{\text {accr }} \approx 4 \times 10^{-2} M_{\odot} /$ s. Since at the same time the neutron star mass changes at a rate of $\dot{M}_{\mathrm{ns}} \approx 1.1 \times 10^{-2} M_{\odot} / \mathrm{s}$, only a fraction of $\sim 25 \%$ of the infalling matter is actually integrated into the neutron star. The remaining $75 \%$ are heated and reejected with high velocity in a neutrino-driven wind that inflates a buoyant 
bubble of neutrino-heated gas in the northern hemisphere opposite to the remaining accretion funnel on the other side (see Fig. 2, lowermost left panel). The resulting flow, which is characterised by a strong dipole mode, can be conveyed only incompletely with plots such as Fig. 2 and is much more impressively captured by movies that we have produced from our data ${ }^{1}$.

These movies also show that the impact and rapid deceleration of the accretion streams in the vicinity of the forming NS create acoustic and weak shock waves that emanate from the neutron star's surface. As was recently also noted by Burrows et al. (2006), these waves propagate predominantly into the hemisphere opposite to the accretion funnel. While traversing the low-density cavity, also the acoustic waves steepen into shocks, dissipate energy, and heat the expanding material. However, in accordance with the results of Janka \& Müller (1996), we observe only a modest production of entropy due to these waves when they propagate outward in the rapidly expanding neutrino-driven wind. In case of the Burrows et al. (2006) simulation the acoustic energy input from neutron star g-mode oscillations was found to be crucial for the explosion of an $11 M_{\odot}$ model. Nonspherical accretion was found to lead to the excitation of core g-mode oscillations at late times ( $\gtrsim 300-500 \mathrm{~ms})$ after bounce, whose sonic damping transfers a significant amount of acoustic power to the surrounding medium and supernova shock. G-mode oscillations are in fact also present in the outer layers of our neutron stars - i.e. in the layers that are included on our grid - but their amplitudes are modest and thus they do not lead to the strong consequences reported by Burrows et al. (2006). It is possible, however, that our simulations underestimate such effects, which would require the inclusion of the whole neutron star without excising the central core, and the ability to follow the excitation of deep modes due to a selfconsistent coupling between accretion, core motion, and coremode generation. On the other hand, our models are characterized by explosions due to convectively supported neutrino heating (whereas the $11 M_{\odot}$ model of Burrows et al. seemingly did not explode in that way). After the explosion has been launched, our models reveal the development of a strong neutrino-driven wind, in which the dissipation of acoustic waves may have a different effect than in a more or less static gas around the oscillating neutron star. We point out that numerical simulations of this neutrino-driven outflow require very high radial resolution of the steep density gradient near the neutron star surface. We are not sure whether sufficiently fine grid zoning was guaranteed in the simulation by Burrows et al. (2006). A more detailed investigation of such questions is in progress.

\subsection{The influence of the neutrino transport}

The fact that earlier 2D simulations, which were performed with a neutrino light-bulb description (Janka \& Müller 1996; Kifonidis et al. 2003), were not dominated by low-order modes, poses the question to which extent the development of such global asphericity in the flow is sensitive to the treatment of the neutrino transport. Figure 4 shows that our new neutrino transport description yields radial profiles for the sum of the $v_{\mathrm{e}}$ and $\bar{v}_{\mathrm{e}}$ luminosities which deviate markedly from the radiusindependent luminosities used in a light-bulb approach: the luminosities are significantly modified compared to the values imposed at the inner boundary. After some adjustment to the local thermodynamic conditions, which takes place in a few radial zones next to the inner boundary, the luminosities rise steeply in

\footnotetext{
${ }^{1}$ A collection of movies is provided as online material of this article.
}
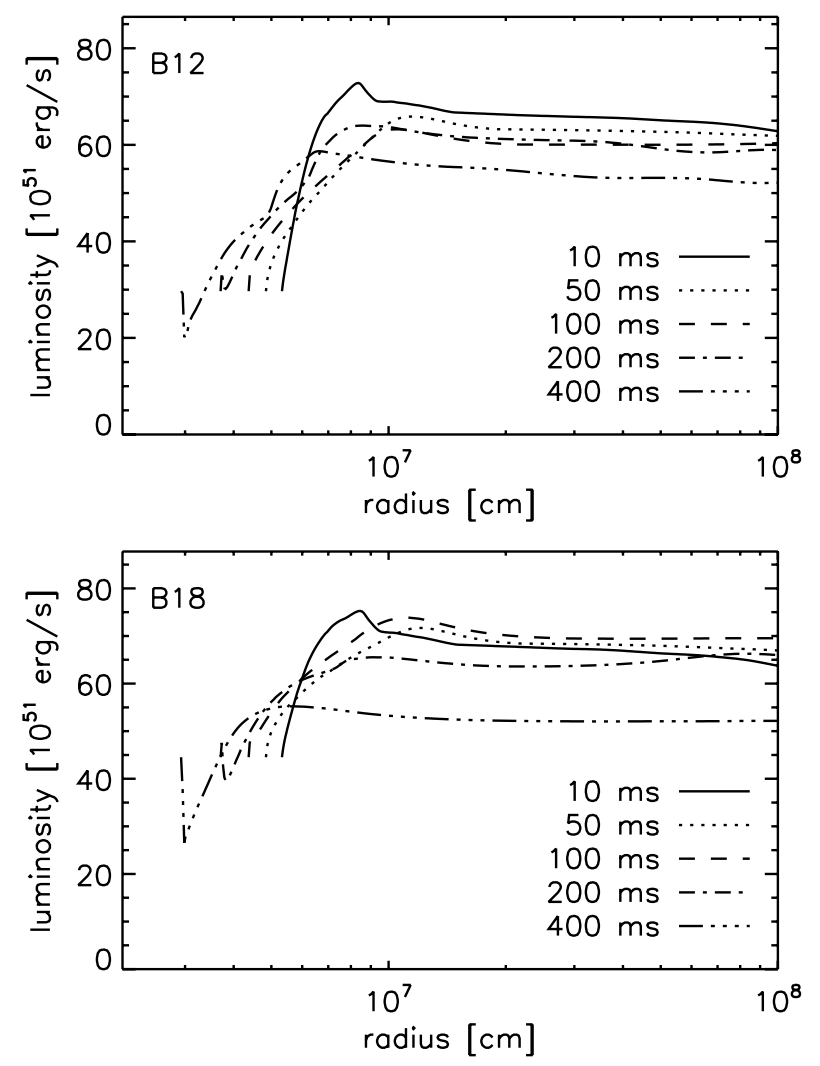

Fig. 4. Radial profiles of the sum of the $v_{\mathrm{e}}$ and $\bar{v}_{\mathrm{e}}$ luminosities for models B12 and B18 at different times after the start of the simulations.

the cooling region below the gain radius, and decline slightly in the heating region farther out. The rise is caused by the creation of neutrinos when gravitational energy is released during the accretion and the contraction of the neutron star, while the slight decline results from the absorption of the $v_{\mathrm{e}}$ and $\bar{v}_{\mathrm{e}}$ in the heating region.

The "accretion" luminosity that is produced on the grid is usually of the same order as the luminosity emerging from the core. In low-energy models, like B12, the accretion component exceeds the core component early on, while in high-energy models the core component is dominant at all times (see the neutrino "lightcurves" for models B12 and B18 shown in Fig. 5).

Our transport scheme can account qualitatively well for the evolution of core and accretion components of the neutrino luminosities, for the radial and temporal evolution of the luminosities and mean energies of the radiated neutrinos, and for the relative sizes of the $v_{\mathrm{e}}$ and $\bar{v}_{\mathrm{e}}$ emission (see Figs. 4-6, and compare with Liebendörfer et al. 2001; Liebendörfer et al. 2005; Rampp \& Janka 2000; Buras et al. 2003, 2006a,b; and Thompson et al. 2003). We therefore think that our current transport treatment is a reasonably good method for performing parametric explosion studies with the aim to better understand the role of hydrodynamic instabilities during the shock-revival phase of neutrinodriven supernova explosions.

Yet, we point out here that all the previously not modelled neutrino transport effects are not the reason why the development of $l=1,2$ modes is seen here, whereas it was not visible in the calculations of Janka \& Müller (1996) and Kifonidis et al. (2003). Highly anisotropic explosions can also be obtained with the light-bulb assumption (see Janka et al. 2003; Janka et al. 2004a for an example). In other words, the details of the functional form of $L(r)$, which are visible in Fig. 4, are not decisive 

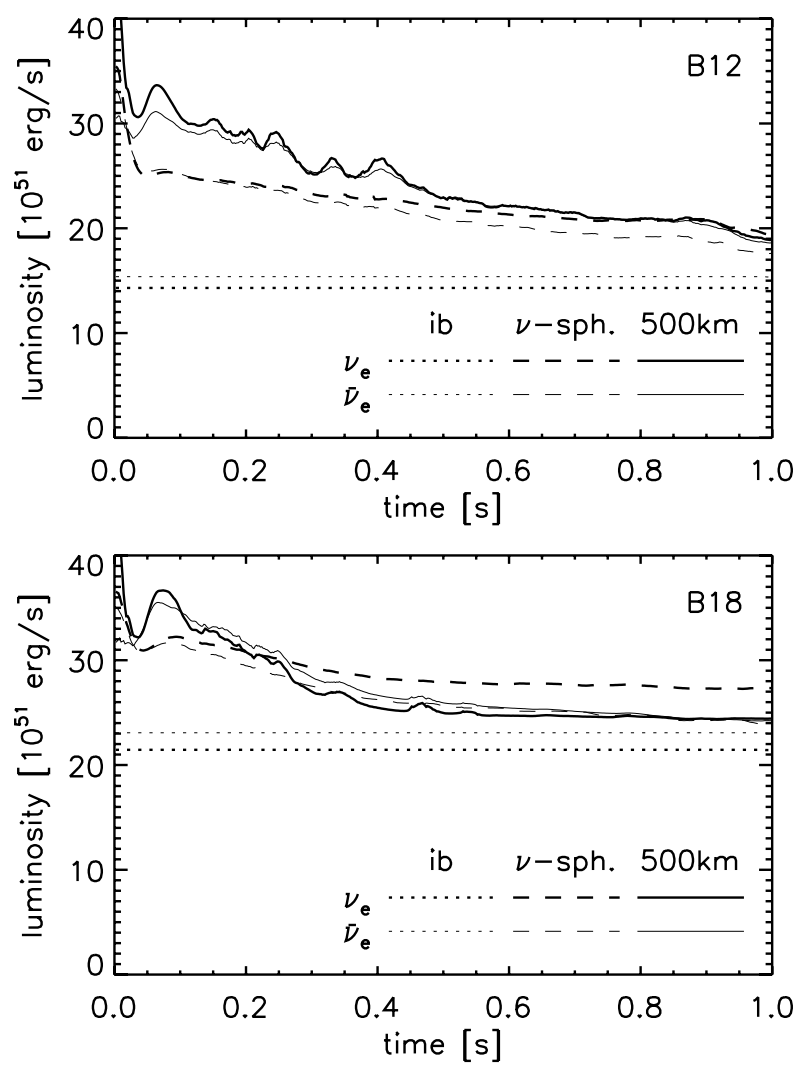

Fig. 5. Evolution of the luminosities of $v_{\mathrm{e}}$ and $\bar{v}_{\mathrm{e}}$ at the inner boundary, at the $v_{\mathrm{e}}$-sphere, and at a radius of $500 \mathrm{~km}$. Note the different importance of the accretion contribution to the luminosity in the low-energy explosion (model B12) compared to the high-energy explosion (model B18) and the rapid decay of the accretion luminosity after the onset of the explosion in the latter model.

for the growth of the $l=1,2$ modes. What is crucial, however, is that the explosions in the current models start slowly. This was not the case in all but one of the simulations of Janka \& Müller (1996) and Kifonidis et al. (2003), where the neutrino luminosities were assumed to decay exponentially with a time scale of typically 500-700 ms (see Table 1 in Janka \& Müller 1996) instead of varying slowly. The exponential, "burst-like" decline of the neutrino light bulb implied fairly high initial luminosities (i.e., $4.5-5 \times 10^{52} \mathrm{erg} \mathrm{s}^{-1}$ for $v_{\mathrm{e}}$ plus $\bar{v}_{\mathrm{e}}$ at the inner grid boundary) - which were required in case of the exponential decay for getting "typical" supernova explosion energies and thus strong neutrino heating occurred at early times after bounce. This led to rapid explosions, which in turn did not leave enough time for the convective cells and bubbles to merge before the expansion became so fast that it continued in a quasi selfsimilar way. Since these bubbles are initially small, their early "freezing out" in the rapidly expanding flow had the effect that small structures (i.e. high-order modes) prevailed until very late times. The rapid explosions also caused a quick end of accretion onto the proto-neutron star, and therefore the neutron stars remained small. In contrast, the present transport description gives neutrino luminosities between the neutrino spheres and a radius of $500 \mathrm{~km}$ that vary much less steeply than exponentially with time (see Fig. 5). This leads to explosion time scales that are sufficiently long to allow for the formation of low-order modes.
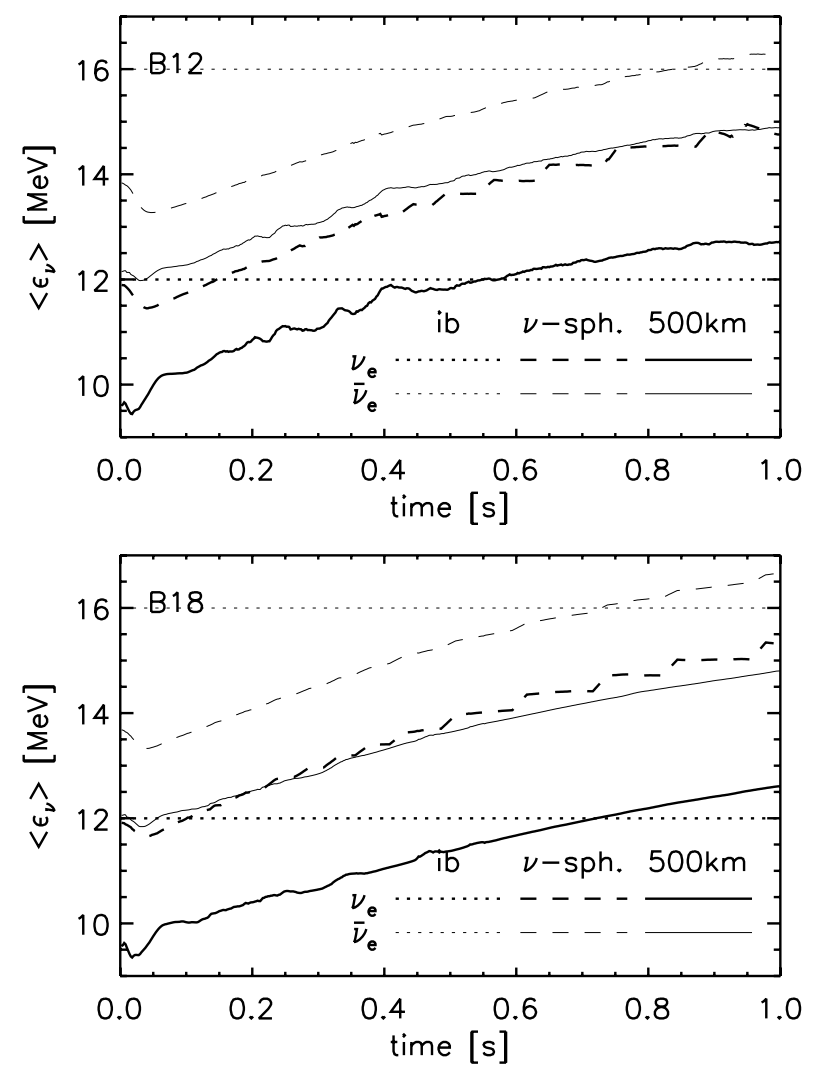

Fig. 6. Evolution of the mean $v_{\mathrm{e}}$ and $\bar{v}_{\mathrm{e}}$ energies at the inner boundary (ib), at the $v_{\mathrm{e}}$-sphere, and at a radius of $500 \mathrm{~km}$. Note that due to the contraction and compressional heating of the nascent neutron star the average energies of the radiated neutrinos continue to rise until the end of our simulated evolution.

\subsection{Acceleration and recoil of the neutron star}

As is detailed in Appendix A, in a 2D axisymmetric calculation the neutron star recoil can only have a component parallel to the $z$-axis. For its calculation only the $z$-momenta of the gas in the northern and southern hemispheres need to be considered (see Eq. (A.20)). If the momentum density of the ejecta, $p_{z}(r, \theta)$, is mirror symmetric with respect to the equatorial plane, i.e., if

$p_{z}(r, \theta)=-p_{z}(r, \pi-\theta)$

holds, the sum of the $z$-momenta of the two hemispheres vanishes

$P_{z, \mathrm{gas}}=P_{z, \mathrm{gas}}^{\mathrm{N}}+P_{z, \mathrm{gas}}^{\mathrm{S}}=0$

and thus also the neutron star remains at rest (cf. Eq. (A.10)). The latter situation is given e.g. for an $l=2$ mode, i.e. for two polar bubbles of equal size separated by a single downflow that is located in the equatorial plane. However, in general the expansion of the ejecta will proceed differently in the two hemispheres, so that a net momentum $P_{z, \text { gas }} \neq 0$ will result.

If a single downflow has formed, e.g., in the southern hemisphere, the expansion of the ejecta will be hampered there. On the other hand it will proceed unaffected in the northern hemisphere, and thus $\left|P_{z \text {,gas }}^{\mathrm{N}}\right|$ will be larger than $\left|P_{z \text {,gas }}^{\mathrm{S}}\right|$. Hence, $P_{z \text {,gas }}$ will be dominated by $P_{z \text {,gas }}^{\mathrm{N}}$ (which is positive since all of the gas in this hemisphere is moving outwards). According to Eq. (A.10), the neutron star must then be accelerated in the negative $z$-direction, i.e. towards the (southern) hemisphere that contains the downflow. This is the situation that ultimately establishes in model B12 (compare Fig. 2 and Table 1), and which 

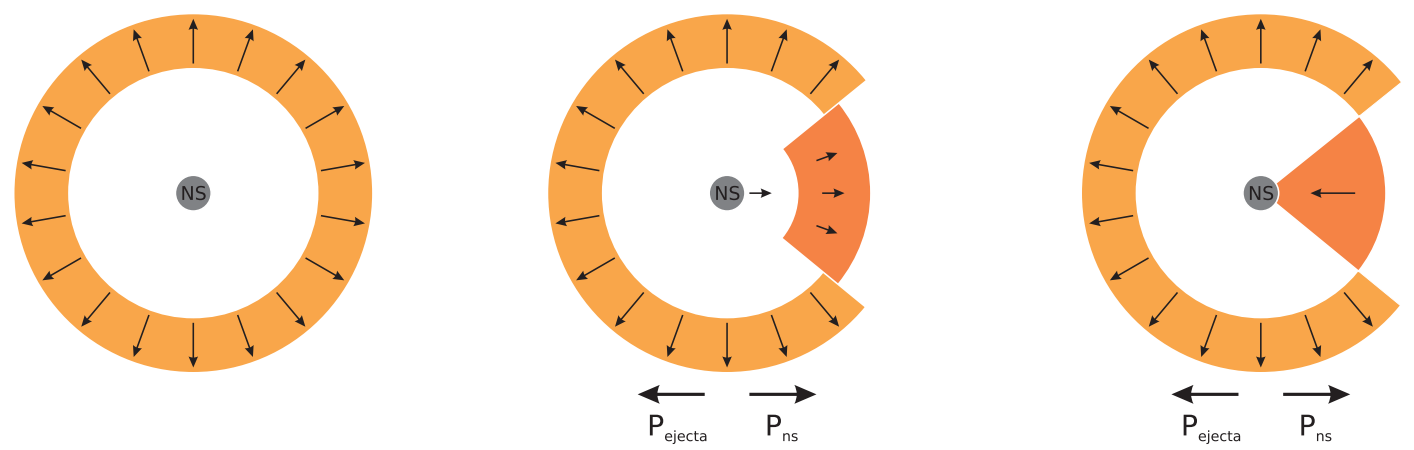

Fig. 7. Graphical illustration of the momentum balance between neutron star and ejecta. The largest fraction of the ejecta mass is concentrated in a dense shell behind the shock (bright coloured ring). For a spherical explosion (left panel) the momenta of the neutron star and the ejecta are zero. If the expansion of one hemisphere lags behind the other, the gas has a net momentum in the direction of the faster expanding hemisphere. The neutron star is always accelerated in the opposite direction, i.e. towards the slower moving gas (middle and right panels). This acceleration can be mediated by the gravitational attraction of the anisotropic ejecta (middle panel). In case accretion flows reach down to the neutron star surface (right panel), additional (hydrodynamic) forces may contribute, but the gravitational force, in general, remains dominant.

Table 1. Integrated momenta of the ejecta in the northern $(\theta<\pi / 2)$ and southern hemispheres, as well as their sum, $P_{z, \text { gas }}$, and the resulting neutron star recoil velocity, $v_{z}^{\text {ns }}$, at $t=1 \mathrm{~s}$.

\begin{tabular}{lccrr}
\hline \hline Model & $P_{z, \text { gas }}^{\mathrm{N}}\left[\frac{\mathrm{gcm}}{\mathrm{s}}\right]$ & $P_{z, \text { gas }}^{\mathrm{S}}\left[\frac{\mathrm{gcm}}{\mathrm{s}}\right]$ & $P_{z, \text { gas }}\left[\frac{\mathrm{gcm}}{\mathrm{s}}\right]$ & $v_{z}^{\mathrm{ns}}\left[\frac{\mathrm{km}}{\mathrm{s}}\right]$ \\
\hline B12 & $1.26 \times 10^{41}$ & $-0.20 \times 10^{41}$ & $1.06 \times 10^{41}$ & -389.3 \\
B18 & $1.77 \times 10^{41}$ & $-3.07 \times 10^{41}$ & $-1.30 \times 10^{41}$ & 515.1 \\
\hline
\end{tabular}

is also illustrated in the right panel of Fig. 7. In this case the neutron star has attained a velocity of $v_{z}^{\mathrm{ns}}=-389 \mathrm{~km} \mathrm{~s}^{-1}$ one second after core bounce, and is still accelerated with $a_{z}^{\text {ns }}=-372 \mathrm{~km} \mathrm{~s}^{-2}$ (Fig. 8).

Model B18 also develops a single downflow, which, however, is located in the northern hemisphere, rather close to the equator. Although this model is clearly less anisotropic than model B12 (which is dominated by an $l=1$ mode), the larger explosion energy and faster expansion result in a larger $\left|P_{z \text {,gas }}\right|$ (Table 1). This leads to a larger neutron star kick of $v_{z}^{\text {ns }}=$ $515 \mathrm{~km} \mathrm{~s}^{-1}$ at $t=1 \mathrm{~s}$, while the acceleration at this time is $a_{z}^{\text {ns }}=290 \mathrm{~km} \mathrm{~s}^{-2}$ (Fig. 8). Note that these values are comparable to the mean pulsar birth velocities derived from observations (see Sect. 7.4), and that they are considerably higher than those found in earlier simulations (e.g. Janka \& Müller 1994). This is mainly due to the low-order modes in our calculations, which result in a larger gas momentum anisotropy, $\alpha_{\text {gas }}$ (cf. Eq. (A.13) for a definition), compared to previous work.

The neutron star velocities shown in Fig. 8 (left panels), as well as their time-derivatives labelled with "derivative" and plotted with solid lines in the acceleration plots of Fig. 8 (right panels), are calculated from the simulation data with our standard post-processing approach by assuming total momentum conservation (see Appendix A). The use of this approach requires a justification, because, numerically, energy and momentum might not be strictly conserved (i.e. up to machine accuracy $)^{2}$. Moreover, momentum conservation can be guaranteed analytically only if the gravitational potential can be written as the solution of a Poisson equation. This is, of course, the case for Newtonian gravity. Yet, for the "general relativistic

2 The energy and momentum conservation properties of neutrinohydrodynamic codes like the employed one are discussed in much detail in Rampp \& Janka (2002) and Marek et al. (2006). potential" of Rampp \& Janka (2002) that we used in the simulations, an equivalent of the Poisson equation cannot be derived (Marek et al. 2006).

Since the neutron star kicks discussed in this work depend on the anisotropic distribution of the ejected gas, we do not expect that the small general relativistic corrections or numerical errors of the mentioned kind can seriously affect the results of our calculations to an extent that unrealistically large values for the neutron star recoil velocities are obtained. This expectation is supported by the fact that we find similarly large neutron star kicks in simulations with Newtonian gravity (see Sect. 7.3). Since the NS recoil estimated from our simulations is a consequence of the anisotropic ejection of mass in the explosion, it is also unlikely to be linked to nonconservation of energy and/or momentum on a small level. In order to provide additional and independent evidence that the neutron star velocities estimated on grounds of the assumption of total momentum conservation are reliable, we check them by verifying the estimated neutron star acceleration as a sum of the different forces that contribute to a momentum transfer to the neutron star.

For this purpose we consider a sphere of radius $r_{0} \approx 1.1 R_{\mathrm{ns}}$ that encloses the neutron star. The time-derivative of the neutron star momentum (and hence the neutron star acceleration at a certain time) can then be obtained by integrating the Euler equation over the volume of that sphere, resulting in

$$
\dot{\boldsymbol{P}}_{\mathrm{ns}} \approx-\oint_{r=r_{0}} \mathcal{P} \mathrm{d} \boldsymbol{S}-\oint_{r=r_{0}} \rho \boldsymbol{v} v_{r} \mathrm{~d} S+\int_{r>r_{0}} G M_{\mathrm{ns}} \frac{\boldsymbol{r}}{r^{3}} \mathrm{~d} m .
$$

Here the individual terms account for the varying pressure $\mathcal{P}$ around the sphere, the flux of momentum through the surface of the sphere, and the gravitational acceleration due to the anisotropic matter distribution outside the sphere. For the gravitational term we assume that the matter distribution inside the sphere is spherically symmetric and that the gravitational potential is Newtonian.

The time evolution of the acceleration corresponding to these terms, calculated from the data of models B12 and B18, is shown in the right panels of Fig. 8. Here the second term has been split into two components associated with momentum flux into ("downflows") and out of the sphere ("outflows"). Also displayed is the sum of all terms (labelled by "total"). Integration over time of the latter quantity yields the dashed velocity curve for $v_{z}^{\text {ns,syn }}$ in the left panels of Fig. 8. This should be compared to the solid curve $\left(v_{z}^{\text {ns }}\right)$ which was computed with our standard 

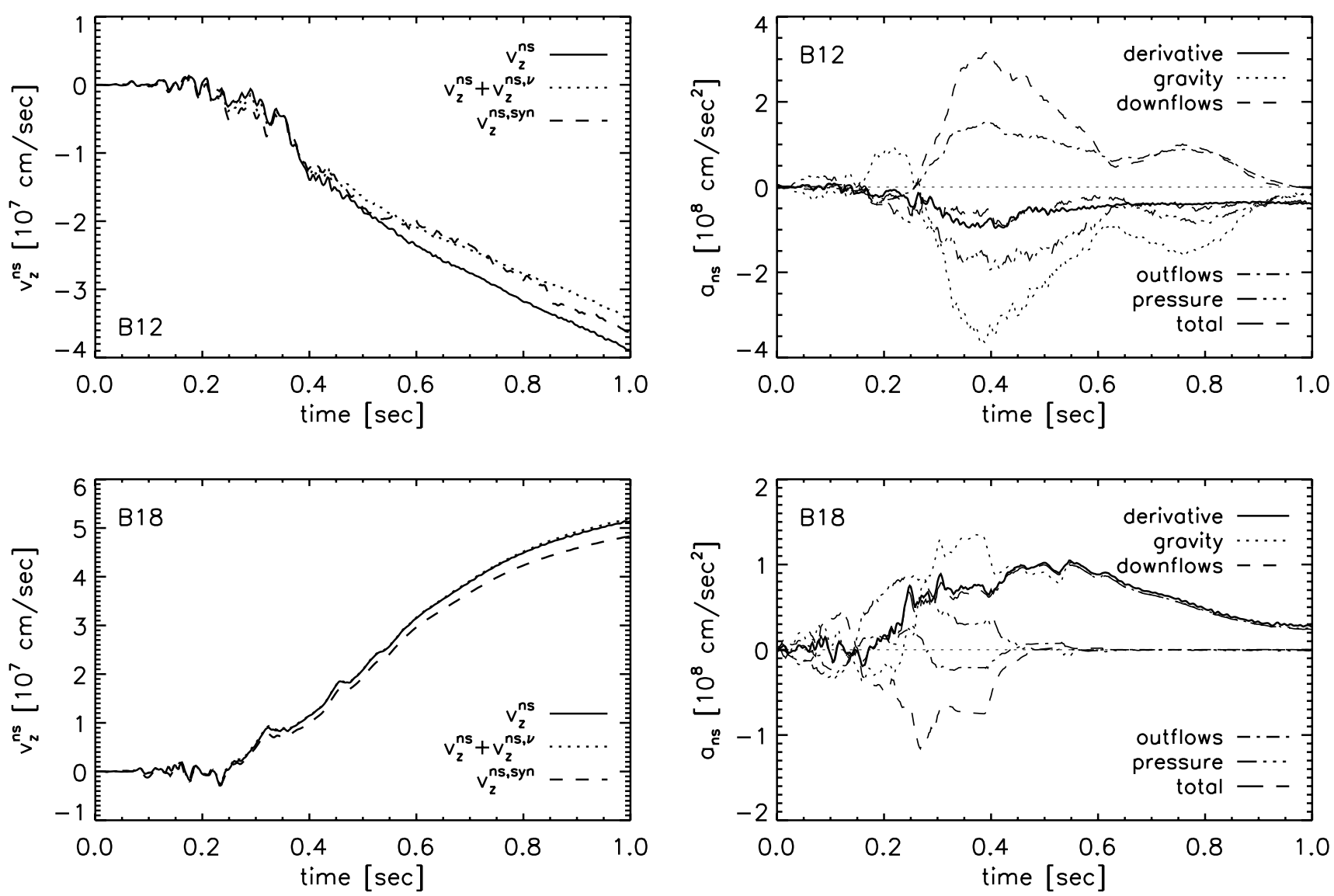

Fig. 8. Left: evolution of the neutron star velocities for models B12 and B18. The solid curve is the neutron star recoil velocity derived from total momentum conservation of gas and neutron star (see Eq. (A.10)). The dotted curve includes corrections due to anisotropic neutrino emission. The dashed curve is an estimate obtained by integrating Eq. (5) over time. Right: evolution of the neutron star acceleration (solid curve), as computed from the (numerical) time derivative of the solid curve shown in the velocity plots on the left side. Also shown are the individual terms of Eq. (5), corresponding to momentum transfer due to downflows, outflows (e.g. in the neutrino-driven wind), anisotropic pressure distribution around the neutron star, and gravitational pull of the anisotropic ejecta. The sum of these terms (the long-dashed curve labelled "total") agrees well with the (solid) curve obtained independently from total momentum conservation applied to the hydrodynamics results.

post-processing approach of the gas momentum (and which includes the effects due to general relativistic corrections). It is evident that there are only small differences between both results, which are significantly smaller than $10 \%$. This demonstrates that the flow morphology indeed produces an anisotropic momentum transfer to the nascent NS, which is the cause of the estimated NS velocities.

An interesting implication of Fig. 8 is the fact that the largest contribution to the acceleration is, in general, due to the gravitational term. In certain evolutionary phases also the other terms may contribute significantly. Yet, the total acceleration points nearly always in the same direction as the gravitational pull. Momentum transfer by pressure and gas flow (the first and the second term in Eq. (5)) are only important as long as the inhomogeneous ejecta have sonic contact with the neutron star and thus can exert hydrodynamic forces on the central object. This is the situation found in model B18 for times before $t \approx 0.5 \mathrm{~s}$. After that time the supersonic neutrino-driven wind, which is very strong in this energetic model (due to the high neutrino luminosities) has blown away the accretion downflows from the neutron star. Ongoing acceleration is then exclusively caused by the gravitational pull of the anisotropic ejecta and decreases slowly as the nearly spherically symmetric wind clears the surroundings of the neutron star. Hydrodynamic forces therefore do not contribute at later times in model B18. On the other hand, they are important at all times in model B12. The acceleration due to the momentum flux associated with the narrow downflows that reach the neutron star is usually the second most important term, and is directed opposite to the gravitational acceleration. Anisotropies in the pressure distribution and wind outflow contribute on a smaller level.

Finally, we show in Fig. 8 (left) with dotted lines the neutron star velocities corrected for the effects of anisotropic neutrino emission (see Appendix A). These effects turn out to be small. For model B12 the neutron star kick is thus reduced by about $10 \%$, which is unusually large. For most of our models (including model B18) the corrections due to anisotropic neutrino emission are smaller than 5\% (for more details, see Sect. 7.1).

\section{Dependence on the initial model and the core luminosity}

In this section we discuss the variation of the quantities introduced in Appendix A as functions of the initial model and a systematic variation of the imposed core neutrino luminosity $L_{\mathrm{ib}}$. Tables A.1-A.5 give an overview. To facilitate their interpretation, we also display the most important quantities for all models as a function of $L_{\mathrm{ib}}$ graphically in Fig. 9. 

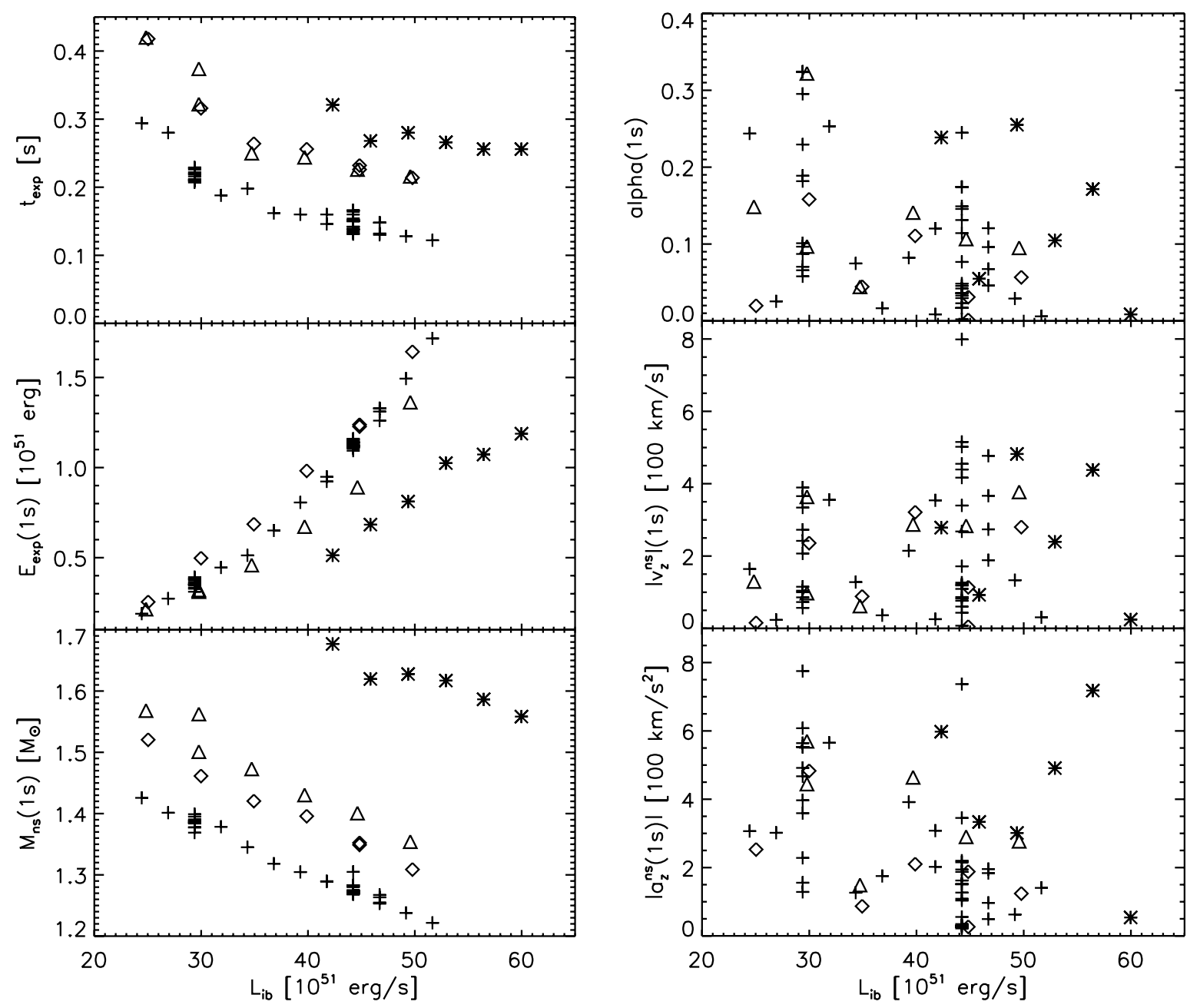

Fig. 9. Dependence of some global quantities on the inner boundary luminosity. The quantities in the left column (explosion time scale, explosion energy, and neutron star mass) depend only on the progenitor and the boundary conditions. The quantities in the right column (anisotropy, neutron star velocity, and acceleration) are strongly influenced by the initial perturbations. All time-dependent quantities are shown at $t=1 \mathrm{~s}$. Crosses stand for the B-series of models, stars mark results for the L-series, triangles denote the W-series, and diamonds refer to the R-series of models (see Sect. 3.1 for the differences between these models).

The results plotted in that figure show that the neutrinodriven mechanism as computed in our models is able to account for different key observational aspects of supernovae and neutron stars simultaneously, provided that sufficient time is available for low-order unstable modes to form. Typical supernova explosion energies of about $10^{51} \mathrm{erg}$, typical baryonic neutron star masses around $1.4 M_{\odot}$ (actually between 1.3 and $1.6 M_{\odot}$ depending on the progenitor) and high neutron star recoils (with a maximum of $800 \mathrm{~km} \mathrm{~s}^{-1}$ in model B18-3 after $1 \mathrm{~s}$ of post-bounce evolution, see Table A.1), are obtained at the same time.

What is also apparent is that the quantities displayed in Fig. 9 can be grouped in two classes, those which show a clear correlation with the core luminosity, $L_{\mathrm{ib}}$, and those which do not. Among the former are the explosion time scale, $t_{\mathrm{exp}}$, the explosion energy, $E_{\text {exp }}$, and the neutron star mass, $M_{\mathrm{ns}}$. For a given initial model these integral quantities show a systematic variation with the boundary luminosity with only little scatter. In particular, these quantities (together with the mean shock radius) are only weakly affected by varying the random seed perturbations in the way described in Sect. 4.1 (see Tables A.1-A.5). Among the quantities which do not correlate with $L_{\mathrm{ib}}$ are the ones that depend on the morphology of the explosion, i.e. the anisotropy parameter, $\alpha_{\text {gas }}$, the neutron star recoil velocity, $v_{z}^{\text {ns }}$, and the neutron star acceleration, $a_{\mathrm{ns}}$. These show a strong sensitivity to small differences in the flow (e.g. to the initial perturbations), and hence essentially stochastic behaviour. The large scatter of neutron star recoil velocities for models B18-1 to B18-6 of between $\sim 80 \mathrm{~km} \mathrm{~s}^{-1}$ and $800 \mathrm{~km} \mathrm{~s}^{-1}$ (see Table A.1) illustrates this clearly.

A higher luminosity, $L_{\mathrm{ib}}$, from the neutron star core causes the explosion to develop faster, to become more energetic, and to leave behind a neutron star with a smaller mass, because less material can be accreted onto the core when the explosion occurs faster. The monotonic correlation between $L_{\mathrm{ib}}$ and the explosion energy $E_{\exp }$ shows that our chosen approach to parameterise our simulations can also be interpreted as one in terms of explosion energy. In this sense $L_{\mathrm{ib}}$ and $E_{\text {exp }}$ can be exchanged as governing parameters. Note, however, that the $L_{\mathrm{ib}}-E_{\mathrm{exp}}$ relation differs between the initial models.

A similar behaviour is also visible in Fig. 10 for $\Delta M_{\text {gain }}\left(t_{\text {exp }}\right)$, the mass contained in the gain layer at time $t_{\exp }$, as a function of $L_{\mathrm{ib}}$ for all models. In fact, it is actually $\Delta M_{\text {gain }}\left(t_{\exp }\right)$ that is responsible for the progenitor dependence of the $L_{\mathrm{ib}}-E_{\text {exp }}$ relation visible in Fig. 9, mainly because the recombination of free nucleons to $\alpha$ particles and nuclei in the expanding and cooling ejecta from the gain layer yields a significant fraction of the 


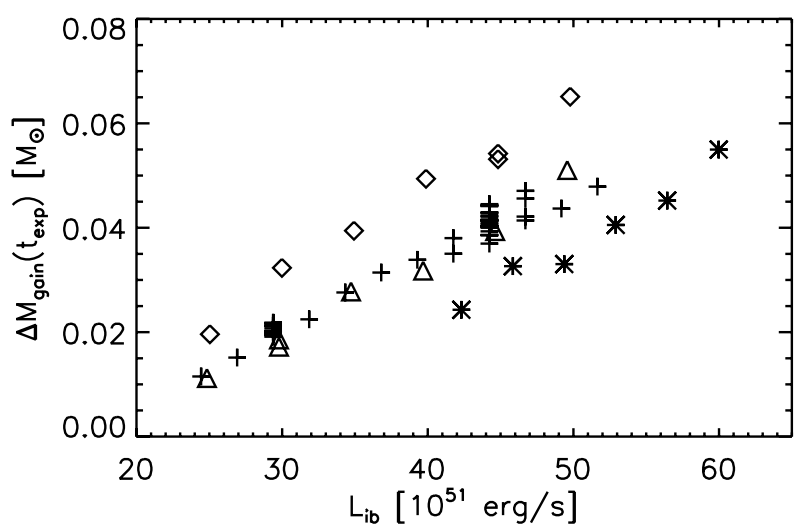

Fig. 10. Mass of the gain layer at the onset of the explosion $\left(t_{\exp }\right)$ as a function of the boundary luminosity for the set of models displayed in Fig. 9. For every initial model there exists an approximately linear relation between $\Delta M_{\text {gain }}$ and $L_{\mathrm{ib}}$.

final explosion energy. This energy contribution increases with more mass in the gain layer. The rest of the explosion energy is due to the power of the neutrino-driven wind of the protoneutron star (see Appendix C). Since $\Delta M_{\text {gain }}\left(t_{\exp }\right)$ depends on the mass accretion rate through the shock, there is a dependence on the density profile of the progenitor star. The different initial models reveal significant differences in this respect. In particular, the Limongi et al. progenitor exhibits considerably higher densities at the edge of the iron core and in the silicon shell than the Woosley et al. models, but this progenitor explodes later and thus at a time when the mass accretion rate has already decreased significantly.

It should be noted that rotation will also affect $\Delta M_{\text {gain }}\left(t_{\text {exp }}\right)$ (see Sect. 6). The systematically larger mass of the gain layer (Fig. 10), and the up to $\sim 50 \%$ higher explosion energies of the rotating models compared to the non-rotating models of the s15s7b2 progenitor (Fig. 9), though, are strongly affected by the larger initial perturbations that we have used in the rotating case (see Sects. 3.1 and 6).

A progenitor dependence is also visible in case of $t_{\exp }$ and $M_{\mathrm{ns}}$ as a function of $L_{\mathrm{ib}}$, as displayed in the left column of Fig. 9. The simulations that are based on the newer $15 M_{\odot}$ progenitor model s15s7b2 of Woosley \& Weaver (1995) give explosion time scales that are systematically higher by $\sim 30 \%$, and final neutron star masses that are higher by $\sim 10 \%$ than those of the older Woosley et al. (1988) core. On the other hand, the results belonging to the Limongi et al. (2000) progenitor again exhibit larger systematic deviations from those for the Woosley et al. stars. The higher mass accretion rate in simulations with the Limongi et al. progenitor delays the development of convective motions, and thus the onset of the explosion $\left(t_{\exp }\right)$ compared to the other models. This prolongs the time that the revived bounceshock needs to reach a certain radius. It also reduces the explosion energy, and leads to a larger neutron star mass, for a given value of the boundary luminosity $L_{\mathrm{ib}}$.

We focus now on the right column of Fig. 9. Recalling the highly nonlinear, chaotic hydrodynamic evolution in response to a variation of the initial perturbations described in Sect. 4.2, one can understand that there is no clear correlation between $L_{\mathrm{ib}}$ and the quantities $\alpha_{\text {gas }}, v_{z}^{\text {ns }}$, and $a_{z}^{\text {ns }}$, which depend on the explosion morphology. When, however, $\alpha_{\text {gas }}$ is plotted as a function of the explosion energy (see Fig. 11), it becomes apparent that the area near the upper right corner in the $\alpha_{\text {gas }}-E_{\text {exp }}$ diagram, satisfying

$\alpha_{\text {gas }} / \alpha_{0}+E_{\exp } / E_{\text {exp }, 0}>1$
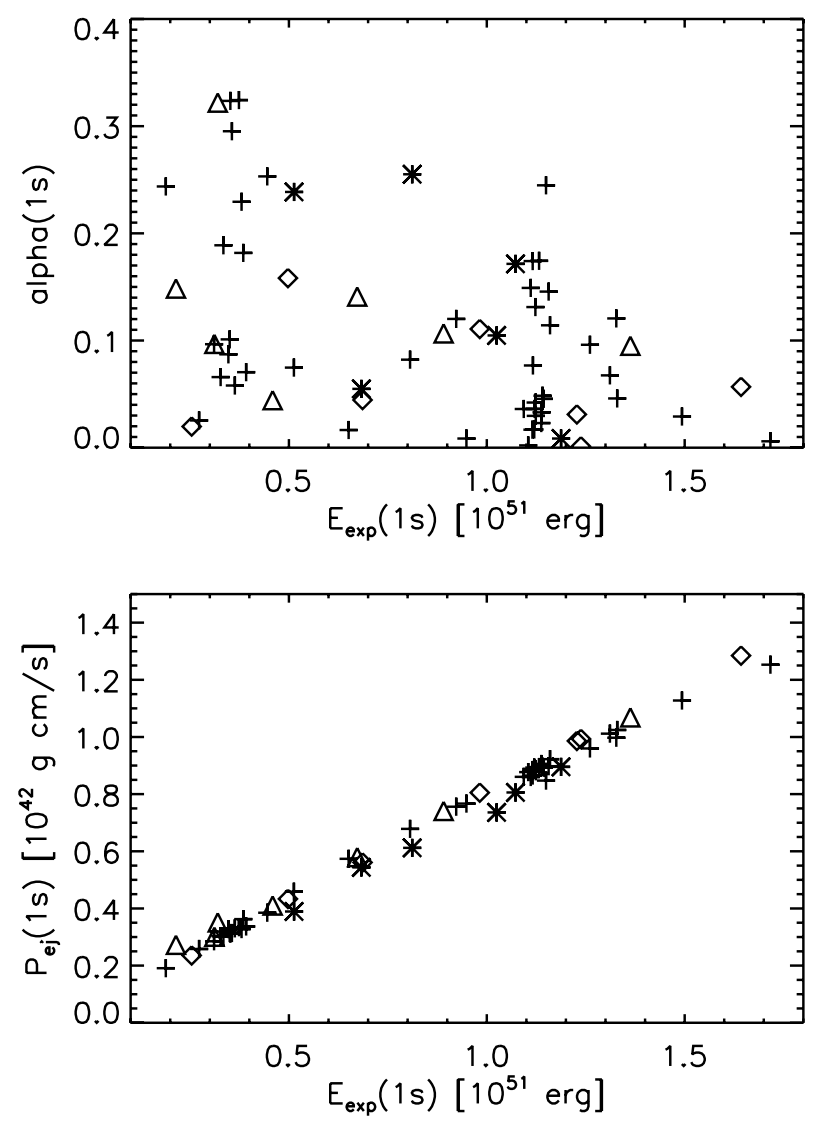

Fig. 11. Anisotropy parameter $\alpha_{\text {gas }}$ (upper panel) and (scalar) quasimomentum of the ejecta, $P_{\text {ej }}$, (lower panel, see Eq. (A.12)) for a time of $1 \mathrm{~s}$ after core bounce as a function of the explosion energy. The different symbols have the same meaning as in Fig. 9.

with $E_{\mathrm{exp}, 0} \approx 2 \times 10^{51} \mathrm{erg}$ and $\alpha_{0} \approx 0.3$, is almost void. This indicates that high-energy explosions with large anisotropies are disfavoured, which is plausible because there is not sufficient time available for high-order modes to merge. In order to assess the impact of this result on the neutron star recoil by virtue of Eq. (A.14), we need to consider also the scalar quantity $P_{\mathrm{ej}}$, which is defined in Eq. (A.12). Figure 11 shows that it is linearly increasing with the explosion energy. Since $\left|v_{z}^{\mathrm{ns}}\right| \propto \alpha_{\mathrm{gas}} P_{\mathrm{ej}}$, this increase of $P_{\text {ej }}$ with $E_{\text {exp }}$ will tend to compensate the smaller values of $\alpha_{\text {gas }}$ for higher explosion energies. Therefore high neutron star velocities (up to $800 \mathrm{~km} \mathrm{~s}^{-1}$ at $t=1 \mathrm{~s}$ ) can result for a wide range of explosion energies, or, equivalently, boundary luminosities (cf. Fig. 9). Indeed we see that neither bipolar oscillations nor the dominance of an $l=1$ mode are excluded when the explosion energy is moderately large. We expect, however, that for sufficiently large boundary luminosities the explosion time scale, and correspondingly $\alpha_{\text {gas }}$, will become so small that the neutron star velocities will remain low for (very) large explosion energies.

An important result of the present work is that neutron stars that have attained high velocities at $t=1 \mathrm{~s}$ typically experience very high accelerations, too (reaching up to more than $700 \mathrm{~km} \mathrm{~s}^{-2}$ ). This becomes apparent in the panels of Fig. 12, which display the acceleration at the end of our simulations (top) or averaged over the last half of a second, respectively, as a function of the neutron star recoil velocity. In fact two populations of models may be discriminated, a low-velocity, low-acceleration component and a second component extending to much higher 

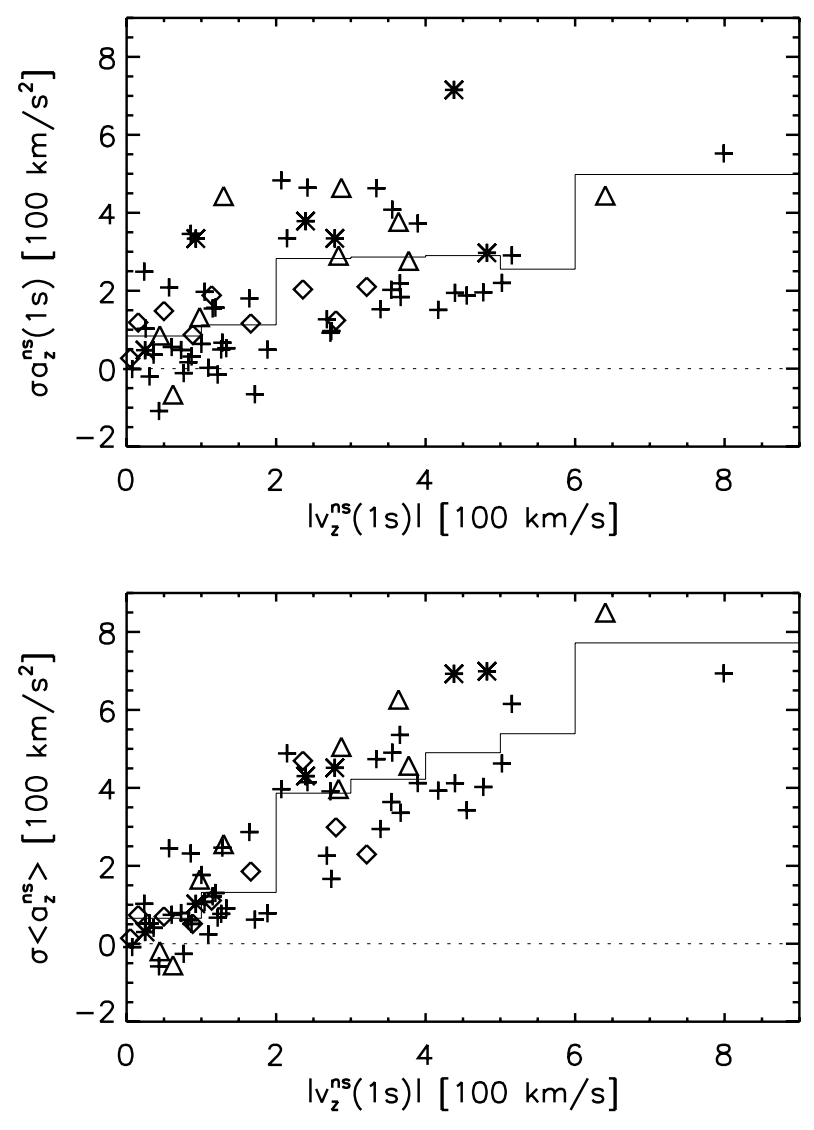

Fig. 12. Top: neutron star acceleration as a function of the neutron star velocity after one second. Bottom: acceleration computed as timeaveraged value over the last half of a second of the simulations versus neutron star velocity. The acceleration is multiplied by a factor $\sigma=\operatorname{sign}\left(v_{z}^{\text {ns }}\right)$, i.e. $\sigma\left\langle a_{z}^{\text {ns }}\right\rangle<0$ corresponds to a deceleration of the neutron star. The different symbols have the same meaning as in Fig. 9. Typically, low values of the acceleration $\left(\sigma\left\langle a_{z}^{\text {ns }}\right\rangle \lesssim 250 \mathrm{~km} \mathrm{~s}^{-2}\right)$ are associated with low velocities $\left(\left|v_{z}^{\mathrm{ns}}\right|<200 \mathrm{~km} \mathrm{~s}^{-1}\right)$, while much higher values of $\sigma\left\langle a_{z}^{\text {ns }}\right\rangle$ are reached for higher velocities $\left|v_{z}^{\text {ns }}\right|$. This suggests two components of the distribution, one with low velocities and lower average acceleration values and one with both values being higher. The thin solid line indicates the mean values of $\sigma\left\langle a_{z}^{\text {ns }}\right\rangle$, binned in velocity intervals of $100 \mathrm{~km} \mathrm{~s}^{-1}$.

accelerations and velocities. The latter contains simulations with a strong contribution of the $l=1$ mode, whereas the former is made up of models in which $l=2$ or higher modes are dominant. Since in many of the simulations the accelerations are still high at $t=1 \mathrm{~s}$, one can expect that their neutron star recoil velocities will significantly increase at still later times. We will discuss this in Sect. 7.4.

\section{The effects of rotation}

We have shown that the magnitude of the neutron star recoil depends sensitively on the convective mode. Here we will consider the influence of rotation, which can have an effect on the pattern of convection via the Høiland condition, which states that the flow is stable to convection if

$$
\begin{aligned}
& C_{\mathrm{H}}:=C_{\mathrm{S}}+C_{\mathrm{L}} \\
& =\frac{1}{x^{3}} \frac{\mathrm{d} j_{z}^{2}}{\mathrm{~d} x}+\frac{1}{\rho} \boldsymbol{a} \cdot\left\{\left(\frac{\partial \rho}{\partial S}\right)_{P, Y_{\mathrm{e}}} \nabla S+\left(\frac{\partial \rho}{\partial Y_{\mathrm{e}}}\right)_{P, S} \nabla Y_{\mathrm{e}}\right\}>0,
\end{aligned}
$$

holds (see e.g. Tassoul 1978). Here $\boldsymbol{a}$ is the total (gravitational and centrifugal) acceleration, and $j_{z}$ is the specific angular momentum $\left(j_{z}=x \cdot v_{\phi}\right.$, where $x=r \sin \theta$ is the distance from the axis of rotation). In the non-rotating case the condition of Eq. (7) reduces to the familiar Ledoux criterion for stability, $C_{\mathrm{L}}>0$, whereas for negligible entropy- and $Y_{\mathrm{e}}$-gradients Eq. (7) becomes the Solberg-condition $C_{\mathrm{S}}>0$.

In order to investigate how rotation changes the morphology, the energetics of the explosion, and the neutron star recoil velocities, we have computed the R-series of our models. These models start from a post-bounce configuration with a perturbation amplitude of several percent (cf. Sect. 3.1), which is more than an order of magnitude larger than the standard perturbations that we employed in our non-rotating models. Such a large increase of the perturbation amplitude leads to noticeable changes in the explosion time scale and energy. A clean discussion of rotationally induced effects therefore requires recomputing some of the non-rotating models with a higher amplitude of the initial random perturbations. We do this in case of models W12-c and W18-c (see Table 2), in which the same initial perturbations are applied as in models R12-c and R18-c, whose results are also listed in Table 2.

\subsection{Evolution of the rotation rate}

The initial rotation profile that we employ was discussed in detail by Müller et al. (2004) (see also Fig. 1 there). Our choice of this angular velocity profile on the one hand maximises rotational effects in view of the most recent evolution calculations for magnetised rotating massive stars: it yields rotation rates that are more than a factor of two higher in the iron core, and on average a factor of ten higher in the silicon shell than in the calculations of Heger et al. (2004). On the other hand, it avoids sub-millisecond rotation of the newly formed neutron star, which would result for rotation rates that are significantly higher than the $0.5 \mathrm{rad} / \mathrm{s}$ with which our iron core was assumed to rotate prior to collapse. With our "standard" contraction law the protoneutron star spins up due to angular momentum conservation to a maximum angular velocity of about $8 \times 10^{2} \mathrm{rad} / \mathrm{s}$ at one second after core bounce. This corresponds to a rotation period of several milliseconds at the end of our simulations (and close to $1-2 \mathrm{~ms}$ after NS contraction to a radius of $10 \mathrm{~km}$ ).

\subsection{Morphology}

The rotating models evolve almost identically to the non-rotating ones during the first $75 \mathrm{~ms}$ after the start of the calculations. This is so because the Solberg-term, $C_{\mathrm{S}}$, is negligible in this early phase. The total angular momentum and the derivative of $j_{z}$ in the postshock region are initially rather small (Fig. 13). However, the influence of the Solberg term increases with time because there is a positive gradient of $j_{z}$ upstream of the shock, and matter with increasingly large specific angular momentum is advected into the postshock region (see Figs. 13 and 14). Therefore the positive derivative of $j_{z}$ with $x$ grows within the postshock flow. Note that, since we assume axisymmetry, there are no forces (other than fictitious ones) acting in $\phi$ direction, and hence no source terms for $j_{z}$ are present. The specific angular momentum of a fluid element therefore remains constant, and $j_{z}$ is simply carried along with the flow.

For $t>75 \mathrm{~ms}$ this causes the Solberg term to become sufficiently large so that it affects the pattern of convection and thus leads to differences compared to the non-rotating case: all 
Table 2. Rotating and non-rotating models with the same initial perturbations. For more details, see the caption of Table A.1.

\begin{tabular}{|c|c|c|c|c|c|c|c|c|c|c|c|c|c|}
\hline Model & $\begin{array}{r}L_{\mathrm{ib}} \\
{[\mathrm{B} / \mathrm{s}]}\end{array}$ & $\begin{array}{r}\Delta E_{v, \text { core }}^{\text {tot }} \\
{[\mathrm{B}]}\end{array}$ & $\Delta Y_{\mathrm{e}, \text { core }}$ & $\begin{array}{c}\left.L_{500}\right\rangle \\
{[\mathrm{B} / \mathrm{s}]}\end{array}$ & $\begin{array}{r}\Delta E_{500} \\
{[\mathrm{~B}]}\end{array}$ & $\begin{array}{r}E_{\text {exp }} \\
{[\mathrm{B}]}\end{array}$ & $\begin{array}{r}t_{\exp } \\
{[\mathrm{s}]}\end{array}$ & $\begin{array}{r}M_{\mathrm{ns}} \\
{\left[M_{\odot}\right]}\end{array}$ & $\begin{array}{r}v_{z}^{\mathrm{ns}} \\
{\left[\mathrm{km} \mathrm{s}^{-1}\right]}\end{array}$ & $\begin{array}{r}v_{z}^{\mathrm{ns}, v} \\
{\left[\mathrm{~km} \mathrm{~s}^{-1}\right]}\end{array}$ & $\begin{array}{r}a_{z}^{\text {ns }} \\
{\left[\mathrm{km} \mathrm{s}^{2}\right]}\end{array}$ & $\alpha_{\text {gas }}$ & $d_{\text {shock }}$ \\
\hline W12-c & 29.7 & 71.5 & 0.11 & 68.7 & 57.1 & 0.40 & 0.301 & 1.535 & 44.4 & 54.0 & 85.4 & 0.03 & 0.63 \\
\hline W18-c & 44.5 & 107.3 & 0.16 & 79.0 & 61.1 & 1.06 & 0.215 & 1.392 & 640.4 & -8.5 & 444.4 & 0.21 & 0.08 \\
\hline R12-c & 29.7 & 71.5 & 0.11 & 64.8 & 51.1 & 0.43 & 0.329 & 1.480 & 49.9 & 31.3 & 148.1 & 0.04 & -0.03 \\
\hline R18-c & 44.5 & 107.3 & 0.16 & 75.5 & 58.1 & 1.26 & 0.236 & 1.345 & 166.1 & -3.5 & 116.2 & 0.04 & 0.05 \\
\hline
\end{tabular}

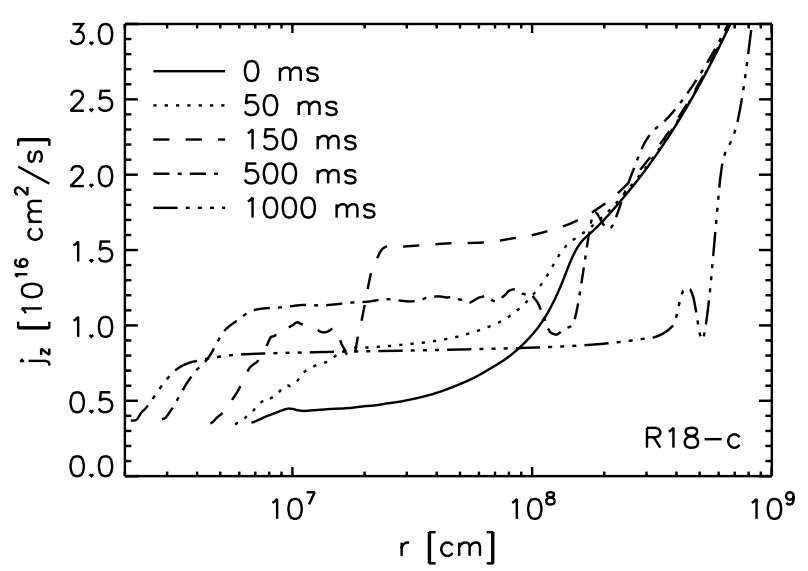

Fig. 13. Radial profiles of the specific angular momentum, $j_{z}$, at the equator of model R18-c for several times after the start of the simulation.

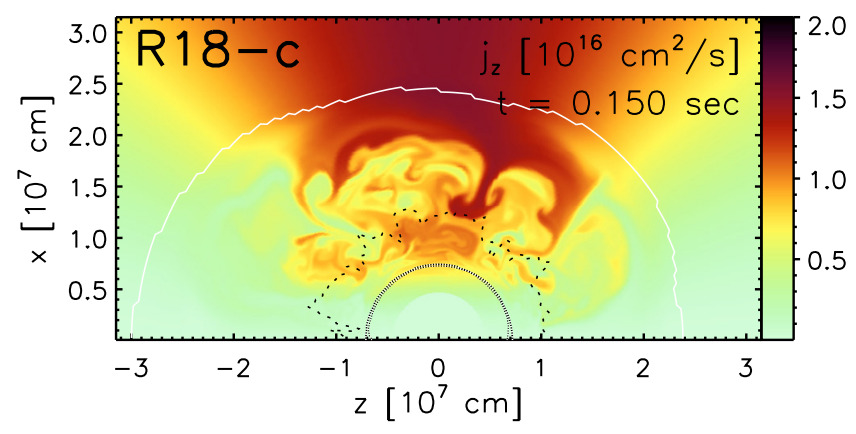

Fig. 14. Distribution of the specific angular momentum $j_{z}$ of the rotating model R18-c at $t=150 \mathrm{~ms}$. Matter with larger and larger specific angular momentum has fallen through the shock (outer solid line), which leads to an overall positive gradient $\mathrm{d}_{z}{ }^{2} / \mathrm{d} x$ in the gain layer. However, due to convection, which is suppressed only near the poles, the $j_{z}$ stratification and its gradient are locally perturbed. The rotation axis is oriented horizontally.

the rotating models develop downflows at both poles, whereas there is no preference for the formation of polar downflows in the non-rotating models (compare Fig. 15 with Figs. 2 and 3). These polar downflows remain stable until they are blown away from the vicinity of the neutron star by the neutrino-driven wind. The stabilisation is caused by the positive $x$-derivative of $j_{z}^{2}$ in the Solberg term, which is amplified by the factor $1 / x^{3}$ near the axis of rotation. Given a positive derivative of $j_{z}^{2}$, a matter element pushed towards the axis feels a larger centrifugal acceleration $a_{\mathrm{c}}=j_{z}^{2} / x^{3}$ than the surrounding matter, and therefore moves back to its original position. Analogously, a fluid element pushed away from the axis feels a restoring force as well. Thus, perturbations perpendicular to the axis are suppressed and perturbations of a gas configuration in rotational equilibrium can only grow parallel to the axis of rotation.

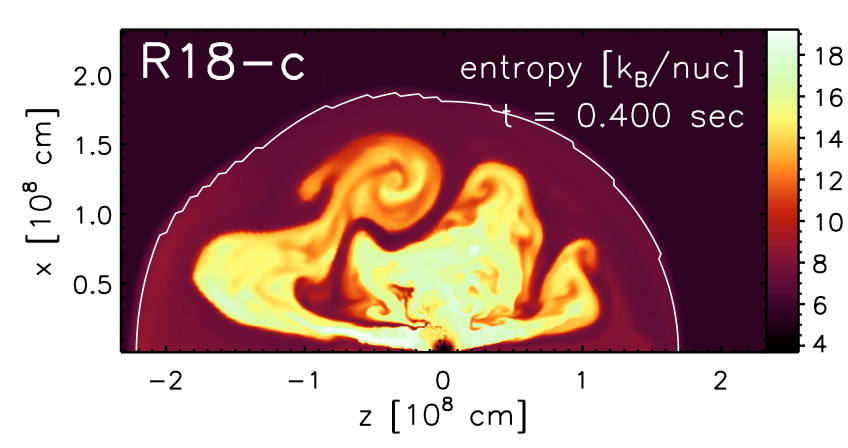

Fig. 15. Entropy distribution of the rotating model R18-c $400 \mathrm{~ms}$ after the start of the simulations. The white line marks the supernova shock. Note the two polar downflows. The rotation axis is oriented horizontally.

For $t>75 \mathrm{~ms}$ this stabilising effect of the positive angular momentum derivative becomes sufficiently large to suppress convection near the axis of rotation, i.e. to make $C_{\mathrm{H}}=C_{\mathrm{S}}+C_{\mathrm{L}}>$ 0 there. In the rest of the postshock flow the Solberg term is negligible (because of its dependence on $x^{-3}$ ) compared to the Ledoux term (i.e. $\left|C_{\mathrm{S}}\right| \ll\left|C_{\mathrm{L}}\right|$ ) and convection is not affected much. Radial profiles of $C_{\mathrm{S}}$ and $C_{\mathrm{L}}$ illustrating this situation are shown in Fig. 16.

The fact that only polar downflows and no polar outflows form can also be easily explained. Material inside a polar downflow always consists of the lowest $j_{z}$-gas that is advected through the shock (see Fig. 14). This guarantees a stable situation because the angular momentum derivative with $x$ remains positive. In contrast, a polar outflow, i.e. a rising polar bubble, would contain postshock matter that would be rather well mixed, because a convective plume encompasses matter from a larger range of latitudes. Therefore such a polar bubble would not consist of gas with a lower $j_{z}$ than the infalling material near the poles that surrounds such a bubble. This situation would therefore be unstable due to the absence of a positive derivative $\mathrm{d} j_{z}^{2} / \mathrm{d} x$.

Besides the differences in the pattern of convection another morphological difference becomes evident: The rotating models remain more spherical, whereas the non-rotating models in general develop a clear prolate deformation (Fig. 17). This is partly due to the polar downflows, which damp the shock expansion near the poles. A second reason is the centrifugal acceleration of the matter between neutron star and shock. Owing to the accumulation of angular momentum behind the shock, the initially weak centrifugal forces increase, and their radial components reach up to $20 \%$ of the gravitational acceleration. Consequently the shock is pushed out farther in the equatorial region than in the non-rotating models. This has interesting consequences for the explosion energy. 

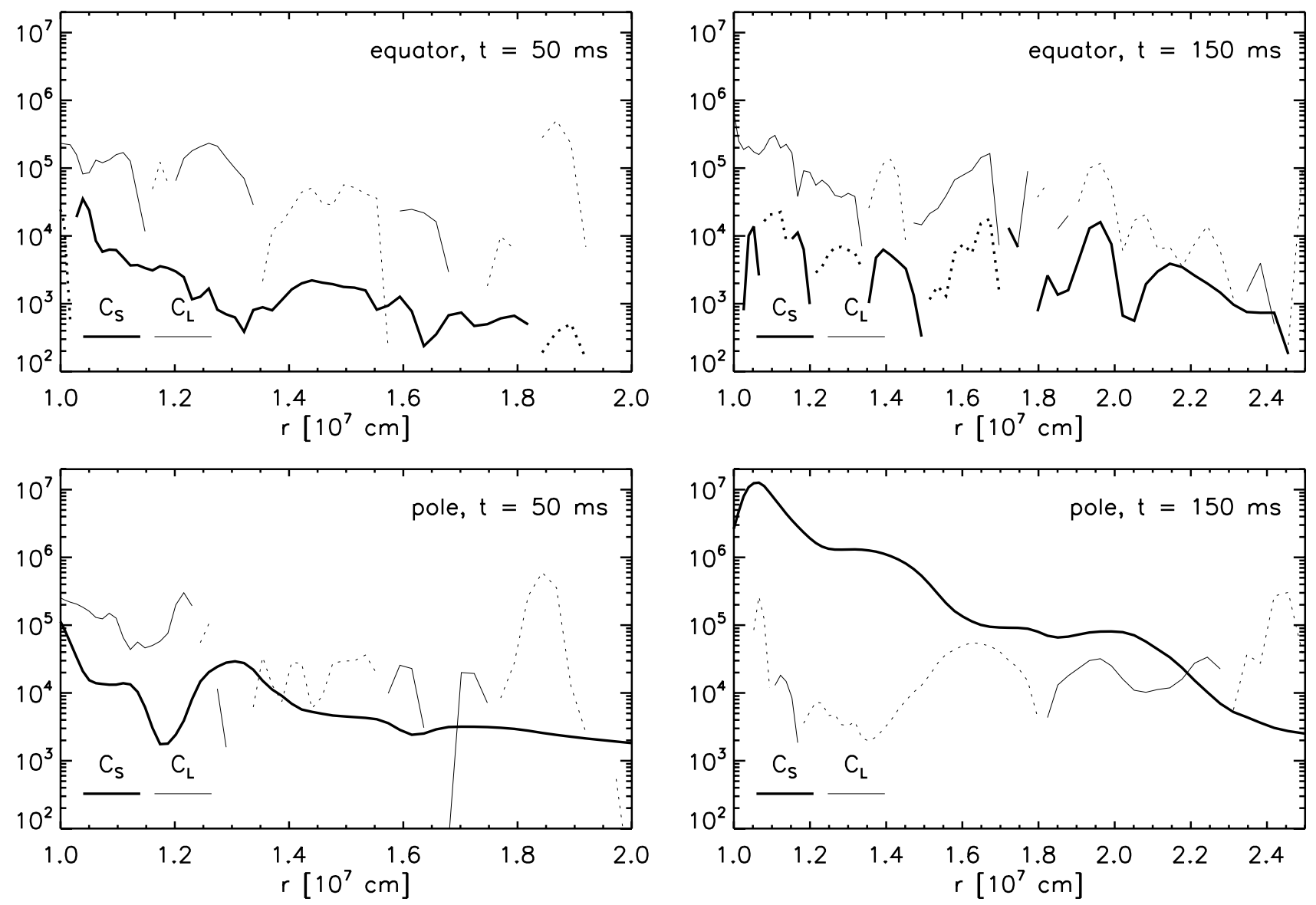

Fig. 16. Radial profiles of the Solberg-term, $C_{\mathrm{S}}$, and of the Ledoux-term, $C_{\mathrm{L}}$, (see Eq. (7)) for $\theta=5^{\circ}$ ("pole") and $\theta=90^{\circ}$ ("equator") in model R18-c. We show these quantities for $t=50 \mathrm{~ms}$ (left column) and $t=150 \mathrm{~ms}$ (right column). For regions in which $C_{\mathrm{S}}$ or $C_{\mathrm{L}}$ are negative, the absolute values are plotted as dotted lines. At $t=50 \mathrm{~ms}\left|C_{\mathrm{L}}\right|>\left|C_{\mathrm{S}}\right|$ and unstable regions $\left(C_{\mathrm{L}}+C_{\mathrm{S}}<0\right)$ are present for both latitudes. At a time of $150 \mathrm{~ms}$ the gradient $\mathrm{d} j_{z}^{2} / \mathrm{d} x$ has become sufficiently large to make $C_{\mathrm{S}}>\left|\mathcal{C}_{\mathrm{L}}\right|$ at the pole, and thus to stabilise the flow, whereas in the equatorial region $\left|\mathcal{C}_{\mathrm{S}}\right|$ is still small.

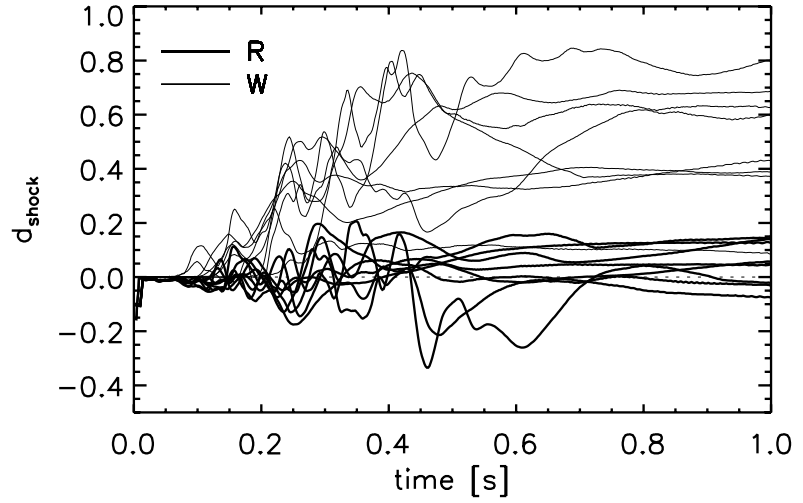

Fig. 17. Evolution of the shock deformation parameter $d_{\text {shock }}$ (see Eq. (A.8)) for the rotating (R-series) and the non-rotating models (W-series). Positive and negative values of $d_{\text {shock }}$ characterise oblate and prolate deformation of the shock, respectively.

\subsection{Energetics}

In both rotating models $\mathrm{R} 12-\mathrm{c}$ and R18-c that are listed in Table 2 the explosion energies are higher and the neutron star masses are correspondingly lower than in their non-rotating counterparts, W12-c and W18-c, also listed in that table. In case of models R18-c and W18-c the energy difference amounts to $\sim 20 \%$ (i.e. $0.2 \times 10^{51} \mathrm{erg}$ ) and must be caused by rotational effects. This difference builds up when the expanding and cooling neutrino-heated matter in the gain layer recombines from free nucleons to alpha particles (and partly to nuclei) and remains approximately constant in the subsequent phase, in which the explosion energy increases further due to the neutrino-driven wind (see Fig. C.6 and Appendix C). It is caused by the larger equatorial shock radius in the rotating model R18-c and the thus wider gain layer, which increases the recombining mass by $0.013 M_{\odot}$ compared to the non-rotating case.

\subsection{Neutron star recoil}

What are the implications of the morphological differences between rotating and non-rotating models for the neutron star kicks? In the non-rotating case the highest recoil was obtained for model B18-3, in which a pronounced $l=1$ mode with a single polar downflow is present. In the rotating case such a flow pattern cannot establish, since we always obtain downflows at both poles. However, significant asymmetries can still develop, since one of the polar accretion funnels may be much stronger than the other, or a third downflow may be dominating the mass distribution. High neutron star recoils are thus not precluded, 
although we expect the mean and the maximum kicks to be somewhat smaller than in the non-rotating case.

The results of our rather few simulations, which comprise only nine rotating models (see Tables A.4 and 2), are in agreement with this expectation: The largest neutron star recoil velocity obtained in the R-series of models is $321 \mathrm{~km} \mathrm{~s}^{-1}$, whereas it is $640 \mathrm{~km} \mathrm{~s}^{-1}$ in case of the W-series (see model W18-c in Table 2). The average kick velocities for the R- and W-type models are $151 \mathrm{~km} \mathrm{~s}^{-1}$, and $280 \mathrm{~km} \mathrm{~s}^{-1}$, respectively. If one omits model W18-c, the only W-type model with a "pure $l=1$ mode", the average kick velocity of the non-rotating models decreases to only $228 \mathrm{~km} \mathrm{~s}^{-1}$, i.e. it is $50 \%$ larger than that of the rotating models. This is a relatively moderate effect if one recalls that the initial angular velocity assumed in the progenitor core of our calculations is clearly extreme compared to the rotation rates obtained from the latest stellar evolution calculations (Heger et al. 2004).

\subsection{Spin-kick alignment?}

Does rotation lead to an alignment of the kick direction with the rotation axis (the so called "spin-kick alignment")? This question cannot be conclusively answered on the basis of 2D axisymmetric simulations, because in this case the neutron star kick is always along the rotation axis due to the assumed symmetry of the calculations.

However in the context of our kick scenario also in the 3D case effects can be imagined that may lead to a spin-kick alignment. On the one hand, the rotation axis is a preferred physical direction of the system such that the development of global anisotropies (e.g. polar accretion and outflow, bipolar oscillations) might be favoured along this direction. On the other hand, if the rotation period is smaller than the duration of the neutron star kick by a one-sided, non-axial acceleration (in the corotating frame), then any asymmetry will retain only its component parallel to the spin axis, while the perpendicular component will be reduced or extinguished by rotational averaging.

Our results seem to suggest that the first effect may be the more important one. For the angular momentum present in our models there is a tendency of anisotropies (e.g. of downflows) to develop preferably aligned with the rotation axis. While for the relatively "fast" rotation of our models (in the sense discussed in Sects. 3.1 and 6.1), the second effect may also contribute to produce spin-kick alignment, the influence of rotational averaging will be weaker for slower and possibly more realistic rotation. In case of "slow" rotation, i.e. for spin periods of tens of milliseconds in the nascent neutron star and many hundreds of milliseconds in the neutrino-heated convective postshock layer (which are ten times or more larger than in our models), rotation will be unable to enforce perfect alignment of the directions of kick and spin.

Depending on the amount of angular momentum in the supernova core, the hydrodynamic kick mechanism discussed in this paper therefore allows for both possibilities, spin-kick alignment for rapid neutron star rotation $\left(t_{\mathrm{ns}} \ll 1 \mathrm{~s}\right)$ and misalignment or incomplete alignment for long rotation periods $\left(t_{\mathrm{ns}} \gtrsim\right.$ some $100 \mathrm{~ms})$. This seems to be compatible with recent studies of observational constraints on neutron star kicks for isolated pulsars and for neutron stars in binary systems (Wang et al. 2006), although the interpretation of observations is still ambiguous (Johnston et al. 2005).

\section{Robustness and long-time evolution of the neutron star recoils}

We have seen above that rotation, even if it is noticeably faster than in the most recent stellar evolution models, does not preclude neutron star kicks of several hundred $\mathrm{km} \mathrm{s}^{-1}$. However, we have made a number of approximations in our post-processing analysis and used assumptions in our simulations whose impact on the neutron star recoil still needs to be assessed. In addition, we have stopped most of our simulations at a time of one second after core bounce, when the neutron star acceleration was, in many cases, still high. Hence we need to comment also on the later evolution of the kicks. These issues are discussed in the following.

\subsection{Anisotropic neutrino emission}

The neutron star recoil velocities, $v_{z}^{\text {ns }}$, that are listed in Tables A.1-A.5 are computed from Eq. (A.10), i.e. they do not include the effects of anisotropic neutrino emission. As we show in Appendix A, anisotropic neutrino emission results in a correction, $v_{z}^{\mathrm{ns}, v}$, of the neutron star velocity that is described by Eqs. (A.17) and (A.19). In Sect. 4.4 we have already seen that this correction is small for models B12 and B18. This actually holds for most models. Only in a few cases is $\left|v_{z}^{\mathrm{ns}, v} / v_{z}^{\mathrm{ns}}\right|>10 \%$, and in most of these cases the neutron stars have small recoil velocities (cf. Tables A.1-A.5). The correction due to anisotropic neutrino emission in general reduces the kick. This can be understood from the fact that in most models a single prominent accretion funnel is present. The neutron star recoil caused by gas anisotropies is always directed towards this downflow, while the neutrino emission associated with the "hot spot" created by the downflow on the neutron star surface results in a "neutrinorocket engine" that kicks the neutron star in the opposite direction (this circumstance was observed and discussed before by Fryer 2004). However, the acceleration due to the neutrino emission remains small because the anisotropy parameter of the accretion luminosity is typically only a few per cent.

There are several reasons for that. On the one hand, the neutrino-radiating tip of the accretion downstream is highly unstable and its position varies with time, reducing the neutrino emission anisotropy by temporal averaging. On the other hand there is a projection factor of $\cos \theta$ of the downflow impact polar angle, $\theta$, to be included due to the axial symmetry of our models. This factor also reduces the kick. Finally, the time scale of neutrino energy release from the accreted matter is typically significantly longer (the cooling time scale is of order $10 \mathrm{~ms}$ ) than the time scale that the gas remains compressed in the downflow tips (between 0.1 and $1 \mathrm{~ms}$ ) before it spreads around the neutron star surface. Only very close to the lower end of the downdrafts the density of the gas is so high $\left(\rho \lesssim 10^{11} \mathrm{~g} \mathrm{~cm}^{-3}\right)$ that the neutrino emission is extremely large. During its violent impact on the NS surface, the gas, however, overshoots equilibrium conditions. Once decelerated, it bounces back, reexpands immediately, and wraps around the neutron star at radii considerably larger than the minimal radius of impact. This is mainly due to the fact that the gas comes from far out in the progenitor star and is shock heated during accretion. As a consequence, its entropy is still considerably higher than the entropy of the layers around and inside the neutrinosphere (remember that neutrino cooling during the infall is too slow to cool the gas efficiently). Therefore the gas floats and forms an essentially spherical, highentropy and low-density $\left(\rho \sim 10^{10} \mathrm{~g} \mathrm{~cm}^{-3}\right)$ envelope that radiates neutrinos with significantly lower rates than the dense tips 
of the impinging downflows. A part of the gas is integrated in the cooling layer and in response to the neutrino losses settles rather slowly on the NS, while the other, higher-entropy part is added to the region outside of the gain radius and is neutrinoheated until it is blown away again in the neutrino-driven wind. As a result, our models reveal that only at most $10-15 \%$ of the binding energy of the infalling gas in the downflows are radiated highly anisotropically. A much larger part of the released gravitational binding energy is not emitted in the downflows but from the essentially spherical layer enwrapping the nascent NS and settling on $\mathrm{it}^{3}$. Due to the mass ejection in the wind, the total rate of energy loss in neutrinos is actually significantly smaller than the rate of release of gravitational binding energy corresponding to stationary accretion with the mass infall rate through the downflow.

\subsection{Inertial mass of the neutron star}

In most of our simulations we make the simplifying assumption that the inertial mass of the neutron star is infinite, i.e. the consequences of the neutron star motion are ignored during the hydrodynamic simulation. This assumption is dropped in one set of models that is listed in Table A.5. In these simulations the feedback effect of the neutron star motion is taken into account by changing the frame of reference in every time step, thus allowing the ejecta to move relative to the neutron star instead of following the neutron star motion through the ambient gas (see Sect. 2.3 and Appendices A and B).

Comparing the results obtained from both approaches for a sample of about 30 simulations (which made use of the boundary parameters of models B12 and B18), one sees that any given model, all else being equal, develops different explosion asymmetry and therefore NS kick, although the explosion energy and time scale are very similar (see Tables A.1 and A.5). The ensemble distribution of kick velocities, however, shows little change, and in particular neutron star velocities in excess of $400 \mathrm{~km} \mathrm{~s}^{-1}$ after $1 \mathrm{~s}$ of post-bounce evolution are found regardless of whether the relative motion of the neutron star is included or not.

Inspecting our simulations with and without NS motion, we can actually not discover any obvious differences caused by the moving NS (the reader is invited to have a look at the movies for models B12 and B12-m6 that are provided as online material of this article). We think that there are a variety of reasons for that. In the first place, the neutron star acceleration and velocity are typically rather small, in particular before and just after the explosion is launched when the acceleration is still unsteady (see Figs. 8 and 18). Secondly, the downflow deceleration and impact on the NS surface are so extremely violent and create so much sound wave and shock activity that the small effect of NS motion cannot be discerned from other dynamical effects. Thirdly, the downflows and also the neutrino-driven wind at later stages are so fast $\left(>10000 \mathrm{~km} \mathrm{~s}^{-1}\right)$ and their accelerations so high that the neutron star motion even with hundreds of $\mathrm{km} \mathrm{s}^{-1}$ (but still rather modest acceleration) is only a small correction.

Since the explosions in our models are triggered by neutrino heating, supported by violent hydrodynamic instabilities,

3 It should be noted that our transport approximation, which assumes that the transport equations in radial direction can be solved independently in all angular zones of the grid, has the tendency to overestimate the neutrino emission anisotropy compared to a fully multidimensional treatment. Therefore our "neutrino recoil" is likely to be an upper limit of the corresponding effect rather than an underestimation.

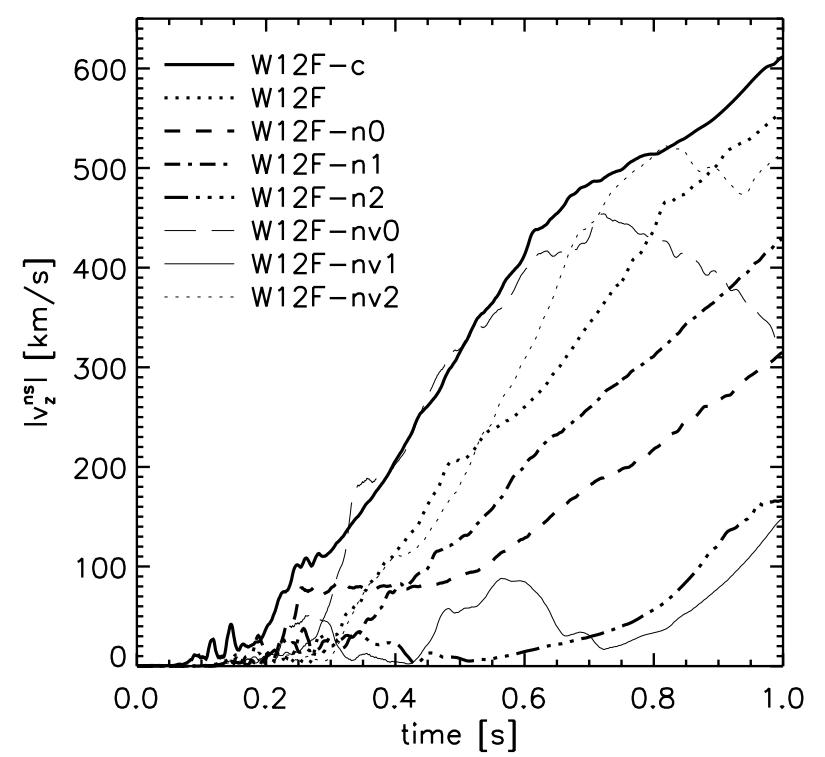

Fig. 18. Neutron star velocities (absolute values) as functions of time for models W12F-c, W12F and several other models with fast neutron star contraction. In six out of eight models the neutron star moves faster than $300 \mathrm{~km} \mathrm{~s}^{-1}$ at $t=1 \mathrm{~s}$.

we suspect that the influence of the neutron star motion might just be masked and dwarfed by other dynamics so that the explosion energy and time scale do not reveal any visible dependence. On the other hand, the nonlinear growth of the hydrodynamic instabilities in the shocked layer is so chaotic that any small changes, independent of their detailed origin (e.g., different initial seed perturbations, different rounding errors on different computers, different neutrino interactions, the moving neutron star, etc.) lead to modifications of the mass and momentum distributions at the end of our simulations. Taking into account the NS motion by our transformation does not have any specific consequences compared to other effects that influence randomness.

\subsection{Neutron star contraction and gravitational potential}

For practical reasons, all simulations listed in Tables A.1-A.5 and Table 2 were performed with our "standard" prescription for the contraction of the neutron star core (see Sect. 3.2), although the "rapid contraction case" also discussed in Sect. 3.2 is potentially more realistic. To study the corresponding differences, we take the "high-perturbation", non-rotating model W12-c (see Sect. 6 and Table 2) as a reference case and perform an additional simulation, model W12F-c, in which we replace the slowly contracting inner boundary of model W12-c with the prescription for a rapidly contracting proto-neutron star. Table 3 compares some quantities characterising the two models.

Model W12F-c explodes earlier and attains a higher explosion energy than model W12-c. This can be explained by the fact that for a smaller inner boundary radius more gravitational energy is released, and that for a shorter contraction time scale this release occurs earlier (see also Appendix C). With $v_{z}^{\text {ns }}(1 \mathrm{~s})=611 \mathrm{~km} \mathrm{~s}^{-1}$ the neutron star recoil velocity of model W12F-c is very high. Large kicks are also found in a set of simulations performed with rapid boundary contraction. For testing this we consider for instance cases with

1. smaller initial random velocity perturbations of $0.1 \%$ (model W12F in Fig. 18); 
Table 3. Important parameters of models W12-c and W12F-c.

\begin{tabular}{|c|c|c|c|c|c|c|c|c|c|c|c|c|c|}
\hline Model & $\begin{array}{r}L_{\mathrm{ib}} \\
{[\mathrm{B} / \mathrm{s}]}\end{array}$ & $\begin{array}{r}\Delta E_{v, \text { core }}^{\text {tot }} \\
\text { [B] }\end{array}$ & $\Delta Y_{\mathrm{e}, \text { core }}$ & $\begin{array}{c}\left\langle L_{500}\right\rangle \\
{[\mathrm{B} / \mathrm{s}]}\end{array}$ & $\begin{array}{r}\Delta E_{500} \\
{[\mathrm{~B}]}\end{array}$ & $\begin{array}{r}E_{\exp } \\
{[\mathrm{B}]}\end{array}$ & $\begin{array}{l}t_{\exp } \\
{[\mathrm{s}]}\end{array}$ & $\begin{array}{r}M_{\mathrm{ns}} \\
{\left[M_{\odot}\right]}\end{array}$ & $\begin{array}{r}v_{z}^{\mathrm{ns}} \\
{\left[\mathrm{km} \mathrm{s}^{-1}\right]}\end{array}$ & $\begin{array}{r}v_{z}^{\mathrm{ns}, v} \\
{\left[\mathrm{~km} \mathrm{~s}^{-1}\right]}\end{array}$ & $\begin{array}{r}a_{z}^{\text {ns }} \\
{\left[\mathrm{km} \mathrm{s}^{2}\right]}\end{array}$ & $\alpha_{\text {gas }}$ & $d_{\text {shock }}$ \\
\hline & .7 & 71.5 & & & 57.1 & .40 & 301 & 1.535 & 44.4 & 54.0 & 85.4 & & 0.63 \\
\hline W12F-c & 29.7 & 101.2 & 0.08 & 110.9 & 62.0 & 0.94 & 0.118 & 1.411 & 611.7 & -1.9 & 580.6 & 0.21 & 0.31 \\
\hline
\end{tabular}

2. a Newtonian gravitational potential and a constant central point mass chosen such that the same initial gravitational acceleration is obtained at a mass coordinate of $1.1 M_{\odot}$ as in the models of Buras et al. (2003), see models W12F-n0, W12F-n1 and W12F-n2 in Fig. 18;

3. a Newtonian gravitational potential and a varying central point mass, which is increased with time to reproduce the evolution of the gravitational acceleration at a mass coordinate of $1.1 M_{\odot}$ in the models of Buras et al. (2003), see models W12F-nv, W12F-nv1 and W12F-nv2 in Fig. 18.

All of these models have in common that they explode more quickly than models with the standard boundary contraction. Yet, for all of these variations we obtain at least one simulation with a neutron star recoil velocity of more than $400 \mathrm{~km} \mathrm{~s}^{-1}$ at $t=1 \mathrm{~s}$ (see Fig. 18). This demonstrates that a faster neutron star contraction does not preclude high neutron star kicks and in particular, it shows that it is not the absolute value of the time scale for the onset of the explosion that matters. What matters is the ratio of the explosion time scale to the growth time scale of low-mode anisotropies by hydrodynamic instabilities like convection, the acoustic-vortex cycle or the SASI mechanism. With the faster shrinking of the neutron star not only the explosion time scale decreases, but also other important conditions change. In particular the advection time scale in the postshock layer and the sound travel time between shock and neutron star become shorter, because the faster NS contraction initially leads to a smaller shock radius, too. Therefore the velocities ahead and behind the stalled shock are higher and the densities in the accretion layer are larger. Since the flow pattern between shock and neutron star surface reacts and adjusts on a hydrodynamic time scale, which is significantly shorter than the contraction time scale of the neutron star, the growth of nonradial instabilities is accelerated in case of shorter dynamical time scales in the accretion layer. Low-mode flow therefore develops faster (more details will be given in Scheck et al., in preparation). Thus the faster NS contraction leads to larger global ejecta asymmetry, in spite of faster explosions. Because of stronger neutrino heating by the higher accretion luminosities the explosion time scales are indeed similarly short as in the light-bulb studies of Janka \& Müller (1994, 1996). The previous "burstlike" light-bulb calculations were actually rather disfavorable for large global anisotropies: due to the high initial luminosities they produced fast explosions, and because of the extended NS the growth of low-mode nonradial instabilities was slow. What therefore finally matters is the ratio of explosion time scale to low-mode growth time scale, and not the absolute period of time in which the explosion develops. The faster growth of non-radial instabilities can result in even higher values of the anisotropy parameter $\alpha_{\text {gas }}$ for "rapid" as compared to "standard" models with the same explosion energy. In other words, the envelope in the $\alpha_{\text {gas }}-E_{\text {exp }}$ plane of Fig. 11 appears shifted towards higher values of $\alpha_{\text {gas }}$ for a faster contraction of the proto-neutron star, and hence also the average recoil velocity (for a specified explosion energy) increases.
In our largest sample of models sharing the same (slowly contracting) boundary condition, i.e. the 18 B18-like models listed in Tables A.1 and A.5, only three simulations develop neutron star recoil velocities of more than $500 \mathrm{~km} \mathrm{~s}^{-1}$, and only seven produce neutron stars with more than $300 \mathrm{~km} \mathrm{~s}^{-1}$ at $1 \mathrm{~s}$. In contrast, in just eight simulations with rapid boundary contraction we obtain six models with neutron star velocities of more than $300 \mathrm{~km} \mathrm{~s}^{-1}$ and three models with neutron stars moving faster than $500 \mathrm{~km} \mathrm{~s}^{-1}$ (Fig. 18). Better statistics would require more simulations, which should also be based on the same initial model ${ }^{4}$ and should make use of the same gravitational potential.

We performed some of the simulations discussed above with Newtonian gravity to demonstrate that the choice of the effective relativistic potential in our models was not essential for our results. We recall that only when we use the Newtonian gravitational potential, momentum conservation can be expected analytically (irrespective of numerical errors and independent of whether the point mass is increased with time, or not). The results therefore show that large neutron star recoil velocities are not linked to any violation of total momentum conservation associated with the use of the effective relativistic potential (see the discussion in Sect. 4.4).

\subsection{Long-time evolution of the neutron star kicks}

In order to investigate how the neutron star recoil velocities evolve beyond a time of one second after core bounce, we perform six exemplary long-time simulations. For these we add 150 radial zones to our grid and place the outer grid boundary at a larger radius of $10^{10} \mathrm{~cm}$, which allows us to simulate the first 3-4 s of the post-bounce evolution. In three of the simulations an infinite inertial neutron star mass is assumed, while in the other models the hydrodynamic feedback of the neutron star motion is taken into account. Four of the six models are just continued from models that we have computed up to a time of one second with our standard grid. We map the corresponding data onto the larger grid at $t=750 \mathrm{~ms}$ and extend the initial model profile from the old to the larger outer boundary of the new grid.

The evolution of the neutron star velocities for all of the long-time simulations is displayed in Fig. 19. The neutron star of model B18-3 is accelerated to more than $1200 \mathrm{~km} \mathrm{~s}^{-1}$ within $3.7 \mathrm{~s}$. This demonstrates that the acceleration mechanism at work in our calculations has the potential to explain even the highest observed pulsar velocities (see e.g. Chatterjee et al. 2005). The fact that model B18-3 is the only one in our sample that produces a neutron star with more than $1000 \mathrm{~km} \mathrm{~s}^{-1}$ does not appear problematic to us. It may be a matter of low-number statistics and might also change when more extreme conditions are realized in models, e.g. by a faster contraction of the neutron star than assumed in our standard set of models. In this respect the sample of simulations plotted in Fig. 18 looks promising. In quite a number of those the neutron stars have large

\footnotetext{
4 The comparison between $\mathrm{B}$ and $\mathrm{W}$ models is viable, however, because both progenitor models are quite similar.
} 


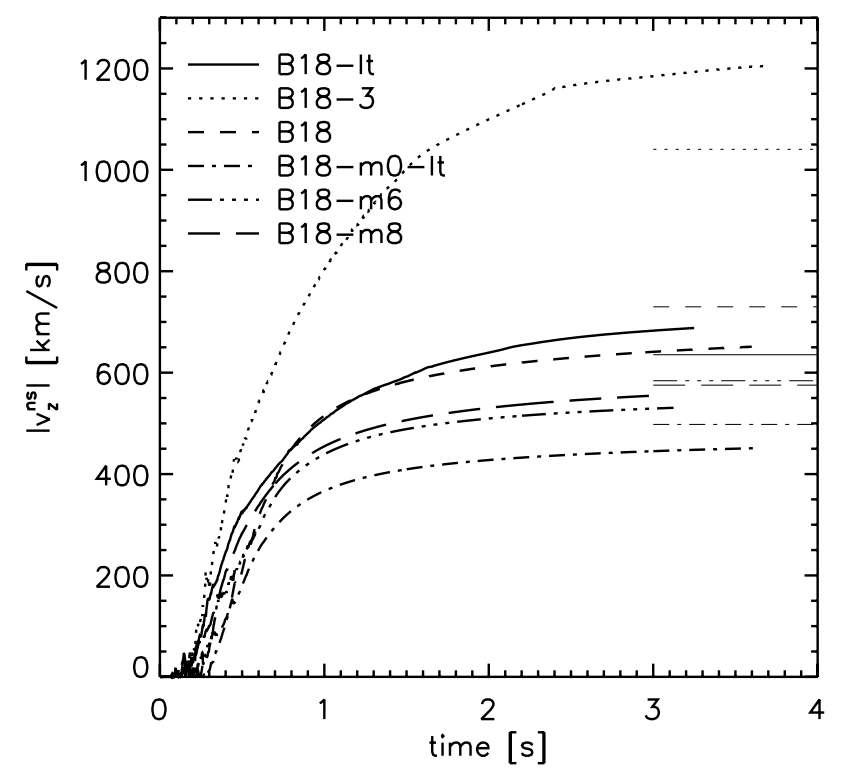

Fig. 19. Evolution of the neutron star velocities in six long-time simulations with the same boundary conditions as model B18. After four seconds the acceleration has become very weak in all models and no significant further increase of the velocities is expected. For each model a thin horizontal line marks the extrapolated velocity value $v_{\mathrm{ns}}^{\infty}$ according to Eq. (8), which is a rough estimate of the final neutron star velocity.

velocities at one second and also still high accelerations (see, e.g., model W12F-c in Table 3).

After 3-4s the neutrino-driven wind has blown away all downflows from the neutron star vicinity and has generated a nearly spherically symmetric wind bubble around it. Therefore the neutron star acceleration diminishes and the recoil velocities approach their terminal values. The latter can be estimated by extrapolating the velocities at $t=1 \mathrm{~s}$, applying an average acceleration value $\left\langle a_{z}^{\text {ns }}\right\rangle$, as computed for the time interval between $t=0.5 \mathrm{~s}$ and $1 \mathrm{~s}$, over a time period $\Delta t_{\text {extrapol}}$, according to

$v_{\mathrm{ns}}^{\infty}=v_{z}^{\mathrm{ns}}(t=1 \mathrm{~s})+\Delta t_{\text {extrapol }} \times\left\langle a_{z}^{\mathrm{ns}}\right\rangle$.

The average acceleration $\left\langle a_{z}^{\text {ns }}\right\rangle$ is introduced as a time-average that is less sensitive to short-time variations and thus allows for a more robust extrapolation of the velocities. The factor $\Delta t_{\text {extrapol }}=0.35 \mathrm{~s}$ is "calibrated" by optimising the estimates in case of the models of Fig. 19. The agreement of extrapolated and computed terminal velocities is typically of the order of $10 \%$. In the following section we use Eq. (8) to estimate the final neutron star velocities for all models listed in Tables A.1-A.5. The basic findings of our analysis do not depend on whether we use $a_{z}^{\text {ns }}$ (the acceleration values at the end of our simulations) or $\left\langle a_{z}^{\text {ns }}\right\rangle$ (the mean values in the last $0.5 \mathrm{~s}$ ) for extrapolating the velocities beyond the simulated period of one second of evolution.

\section{Implications for the neutron star velocity distribution}

In Sect. 5 we pointed out that Fig. 12, showing the neutron star velocities and accelerations at $t=1 \mathrm{~s}$, suggests the existence of two groups of models. One group consists of cases with low velocities and on average low acceleration, and the other group cases with high velocities and significantly higher average acceleration. The latter models are typically characterised by a strong $l=1$ mode in the flow pattern at the end of our simulations.
Provided the acceleration shows a trend of increasing more steeply than linearly with the neutron star velocity, one can expect a growth of the separation of both populations when the acceleration continues over a longer period of time. Thus a bimodal velocity distribution will emerge, caused by the larger acceleration associated with the presence of a dominant $l=1$ mode in the models of the high-velocity group. To test this possibility, we extrapolate the neutron star motions of all of our 70 models listed in Tables A.1-A.5 from one second to the expected final conditions by applying Eq. (8). Figure 20 displays both the velocity distribution at the end of the simulated evolution (at $t=1 \mathrm{~s}$; left panel) and the terminal distribution (right panel).

A comparison of the panels in Fig. 20 shows that most neutron stars of the high-velocity and high-acceleration group (which is indicated by the darker shading) accelerate to significantly higher velocities on time scales longer than one second. In contrast, only very few stars of the low-velocity group reach velocities in excess of $200 \mathrm{~km} \mathrm{~s}^{-1}$. As a consequence, a minimum develops in the extrapolated distribution around $300 \mathrm{~km} \mathrm{~s}^{-1}$, separating clearly the two components in velocity space.

We interpret this result as an interesting demonstration that the kick mechanism discussed here is able to produce a bimodal distribution of neutron star velocities simply due to the presence or absence of a dominant $l=1$ mode in the spatial distribution of the supernova ejecta. Invoking two different processes for neutron star acceleration is not required. It is, however, unclear whether this may provide an explanation of a possible bimodality in the observed velocity distribution of pulsars. The existence of such a bimodality is not only ambiguous, some authors finding hints (e.g. Cordes \& Chernoff 1998; Fryer et al. 1998; Arzoumanian et al. 2002; Brisken et al. 2003), while others favour a one-component Maxwellian distribution (e.g. Lyne \& Lorimer 1994; Hansen \& Phinney 1997; Hobbs et al. 2005; Zou et al. 2005). Also the parameters for the two-component fits differ significantly between the publications.

Though our result is inspiring, as well as tantalising, we refrain from making a direct connection with observations. Such attempts are hampered by the limitations of our analysis, which does not only assume the extrapolation of Eq. (8) to be valid for all cases. Our analysis is also affected by our finding that the magnitude of the neutron star kicks seems to depend on the neutron star contraction (see Sect. 7.3) that is mimicked in our simulations by a moving inner boundary of the computational grid. Moreover, our analysis is constrained to a set of $15 M_{\odot}$ stars $^{5}$, while linking theory with observations would require modelling explosions for a representative distribution of supernova progenitors, making reasonable assumptions about the progenitor dependence of the explosion energy and including the effects from binary breakup. A large set of calculations would have to account for the stochastic nature of the discussed neutron star acceleration mechanism, thus establishing the distribution of kick velocities as a function of the progenitor properties. One might have the concern that in the combined data of all of these runs the minimum visible in the velocity distribution of Fig. 20 is filled up. Finally, quantitatively meaningful calculations of neutron star kicks will ultimately have to be obtained by 3D modelling.

\footnotetext{
5 The employed progenitor models, however, exhibit large differences in core sizes and core density profiles, which actually may be considered as reflecting the variations over a broader range of progenitor masses.
} 

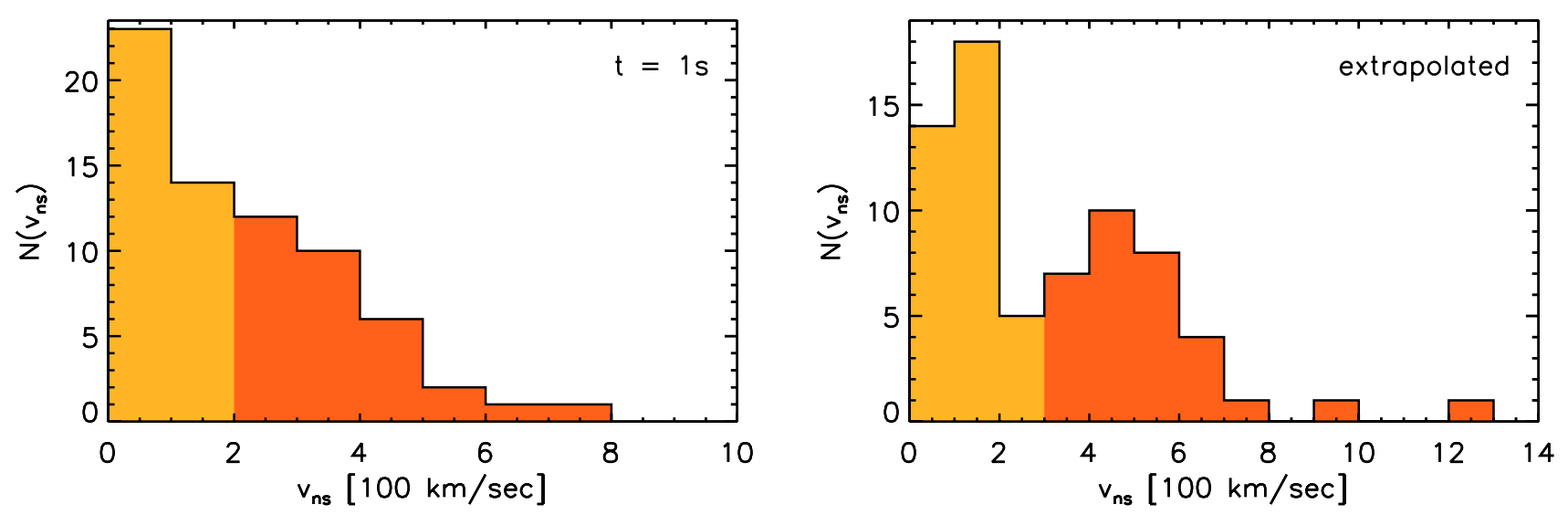

Fig. 20. Histograms of the neutron star velocity distribution for the 70 models of Tables A.1-A.5. The left panel shows the velocity distribution at $t=1 \mathrm{~s}$ (solid black line). The darker shaded area corresponds to the fraction of models whose neutron stars are moving with more than $200 \mathrm{~km} \mathrm{~s}^{-1}$ one second after bounce. The same models are displayed with dark shading also in the right panel, which shows the final velocity distribution as obtained by extrapolation with Eq. (8).

\section{Summary and conclusions}

The aim of this work was to investigate hydrodynamic instabilities in the neutrino-heated postshock layer of core-collapse supernovae and the importance of such instabilities for the development of explosion anisotropies and neutron star kicks.

For this purpose we have presented more than 70 supernova simulations in two dimensions (i.e., assuming axisymmetry) for different $15 M_{\odot}$ progenitor models, relying on the viability of the neutrino-driven explosion mechanism. Since this viability is still an open question and no explosions are obtained in 2D models with a detailed spectral treatment of neutrino transport for stars more massive than about $11 M_{\odot}$ (see Buras et al. 2006a,b), we triggered the explosions in our simulations by replacing the contracting core of the nascent neutron star by an inner boundary of the computational grid and assuming suitable neutrino luminosities there from the neutron star core (see the introduction in Kifonidis et al. 2006, for a motivation and justification of this procedure in light of the results from recent Boltzmann transport supernova simulations). The boundary was placed at a Lagrangian mass coordinate typically of $1.1 M_{\odot}$, where the neutrino optical depths were usually 10 or higher. A systematic variation in the core neutrino luminosities imposed at this boundary allowed us to investigate the growth of hydrodynamic instabilities. This variation also allowed us to study how the explosion and the size of the explosion energy depend on the strength of the neutrino heating.

In contrast to previous work (Janka \& Müller 1996; Kifonidis et al. 2003), the neutrino luminosities of the neutron star core in the models presented here were not assumed to decay exponentially, but - in closer agreement with transport calculations for the whole neutron star - were assumed to remain (roughly) constant on a Lagrangian mass shell of $1.1 M_{\odot}$ over hundreds of milliseconds after the bounce. With this boundary condition, the approximative neutrino transport scheme developed for the present study ensures a radial and temporal behaviour of the neutrino luminosities and mean spectral energies as also found qualitatively in more complete and fully consistent supernova models, i.e. the core and accretion components of the neutrino emission are both accounted for.
Our main results can be summarised as follows.

1. Random perturbations, by which we seed the growth of nonradial instabilities in our simulations, can grow from small initial amplitudes (between $0.1 \%$ and some percent of the fluid velocity) to global asphericities by convective instability, as well as the vortical-acoustic cycle (Foglizzo 2001, 2002), provided the time until the onset of rapid shock expansion is long enough. Once the shock expansion gains momentum, the further growth of the instabilities, e.g. by the merging of smaller structures to larger ones, is quenched, and the flow pattern essentially freezes out. It is not the absolute time until explosion that matters in this context, but the ratio of the explosion time scale to the typical growth time scale of the instability. A detailed investigation of the growth of different kinds of non-radial instabilities in the postshock flow and their competition will be published in a subsequent paper (Scheck et al. 2006, in preparation). The neutrino transport description and employed inner boundary condition for the transport used in this work ensured a sufficient delay of the shock acceleration, in contrast to the light-bulb parameters employed by Janka \& Müller (1996) and Kifonidis et al. (2003).

2. The growth of the instabilities proceeds extremely nonlinearly and chaotically such that the final ejecta anisotropy turns out to be sensitive to the initial random pattern of the seed perturbations, as well as to small differences between numerical runs (connected, e.g., to small changes in the grid zoning, machine roundoff errors, or small differences of the input physics). Despite the different ejecta geometry, however, integral parameters of the models, such as the neutron star mass, explosion time scale, or explosion energy, show little variability.

3. This is different for quantities, which depend on hemispheric asymmetries. The instabilities lead to symmetry breaking, and the ejecta can attain a net linear momentum, balanced by the recoil absorbed by the neutron star. In practise, the momentum exchange was found to be mediated by gravitational, as well as hydrodynamic forces. Typically the former are more important, but in cases where the neutron star accretes anisotropically over long periods of time, hydrodynamic interaction can also contribute significantly. In our 
standard setup for the calculations, the neutron star is fixed (due to the use of the inner grid boundary) at the centre of the grid. Since it therefore does not start moving, in spite of momentum gain, this situation can be considered as a case where the neutron star is assumed to have infinite inertial mass. In order to test whether this affects the results, we performed a number of runs by imposing the negative of the instantaneous neutron star velocity (as calculated from its attained momentum) on the ambient gas on the computational grid. This leads to a collective gas motion relative to the neutron star fixed at the grid centre, and it corresponds to changing the frame of reference by applying a Galilei transformation after every hydrodynamics step. Of course, for any given model, all else being equal, the model with the transformation yields a different explosion asymmetry and a different neutron star kick (but still very similar explosion energy and time scale). But despite these differences in individual simulations, we could not detect any significant changes in the ensemble behaviour with respect to explosion parameters or magnitude and distribution of neutron-star kick velocities.

4. Further tests also showed that the details of the neutrino treatment, the employed gravitational potential (i.e., performing the simulations with Newtonian gravity or an effective relativistic potential according to Marek et al. 2006), the assumed amplitude of initial perturbations, or the assumed contraction of the inner grid boundary (which mimics the shrinking of the cooling nascent neutron star) do not have any qualitative influence on our results for the neutron star kicks. Quantitatively, we discovered indications (based on a limited set of computations, however) that a faster contraction of the forming neutron star - which may correspond to a softer nuclear equation of state or more rapid cooling seems to favour higher neutron star kicks on average. This can be explained by a more rapid growth of low-mode nonradial instabilities, leading to higher values of the aniotropy parameter $\alpha_{\text {gas }}$ for a given explosion energy.

5. While the neutrino flux imposed at the inner grid boundary was assumed to be isotropic in all of our simulations, the neutrino radiation at large distances from the neutron star could become anisotropic because of lateral differences in the neutrino emission and absorption. The biggest such differences are associated with long-lasting, narrow downflows through which the neutron star accretes gas anisotropically. The gas heats up strongly upon falling towards the neutron star surface and getting decelerated in shocks. We found, however, that the corresponding anisotropic neutrino emission produces a neutrino-mediated acceleration that accounts only for small corrections to the neutron star velocities produced by the asymmetric mass ejection. These corrections rarely exceed $10 \%$. Both accelerations usually produce motion in opposite directions. The reason for this is that the neutron star receives a kick towards a downflow (which attracts the neutron star gravitationally or leads to a momentum deficit of the expanding ejecta shell on the side of the downflow), whereas the neutrino radiation is more intense in the hemisphere of the downflow and thus propels the neutron star in the other direction. Since the accretion luminosity is radiated near the neutron star surface, we do not think that our use of the inner boundary underestimates this effect. The inverse is more likely. Our radial transport tends to overestimate the anisotropy of the outgoing radiation, because truly multi-dimensional transport would redistribute the locally emitted neutrinos more isotropically in all directions instead of favouring their radial propagation (see the discussion in Livne et al. 2004).

6. After one second of post-bounce evolution, which was the period of time we simulated for most models, we obtained maximum neutron star velocities up to $800 \mathrm{~km} \mathrm{~s}^{-1}$. The models appear grouped in two populations, one in which the neutron stars move with less than $200 \mathrm{~km} \mathrm{~s}^{-1}$ and have low acceleration at $t=1 \mathrm{~s}$, and another one, roughly equally strong, where the stars have velocities higher than $200 \mathrm{~km} \mathrm{~s}^{-1}$ and on average also higher accelerations (see Fig. 12, and the left panel of Fig. 20). The two groups differ by the absence or presence, respectively, of a strong or dominant $l=1$ dipole mode in the gas distribution around the neutron star. The simulated models cover a range of explosion energies roughly equally between about $0.3 \times 10^{51} \mathrm{erg}$ and more than $1.5 \times 10^{51} \mathrm{erg}$. We could not detect any systematic variations in the typical magnitude or scatter of the kick velocities with the explosion energy. We also did not discover any obvious correlation of the kicks with the properties of the three considered $15 M_{\odot}$ progenitor stars, which exhibited major differences in their core sizes and density structures. Rotation with a fairly high pre-collapse rate of $0.5 \mathrm{rad} / \mathrm{s}$ in the iron core, which in view of the most recent stellar evolution models is probably unrealistically high for ordinary supernovae (see Heger et al. 2004), leads to slightly higher explosion energies (due to a higher mass in the gain layer), a more spherical shock surface, and the presence of downflows at both poles of the rotating neutron star. This suggests a weaker contribution of an $l=1$ mode in this situation compared to the nonrotating models, where downflows in only one hemisphere are rather common. Although very extreme cases were missing in our fairly small sample of simulations with such rapid rotation, we nevertheless obtained kick velocities in excess of $300 \mathrm{~km} \mathrm{~s}^{-1}$ (still rising at one second after bounce), and could not detect any bias towards the group with low velocities and low average acceleration.

7. The two populations in our velocity distribution at one second are certainly interesting in view of the possibility of a bimodality in the distribution of measured pulsar velocities, which however is still controversial. We therefore attempted to derive, from our set of about 70 simulations, the distribution at the time the neutron stars have reached their terminal velocities. In order to do that we continued some of our models until 3-4 s, at which time the accelerations had become very small. These models served for calibrating the typical period of time that a representative acceleration must be applied to extrapolate from the velocity at one second to the terminal values. The representative acceleration was taken as the average value between $0.5 \mathrm{~s}$ and $1 \mathrm{~s}$ after bounce, a choice which guaranteed that short-time fluctuations of the size and direction of the acceleration (which are rather frequent in the case of low-energetic explosions) do not corrupt the extrapolation. Indeed, the extrapolated velocity distribution revealed a clear bimodal structure with a minimum around $300 \mathrm{~km} \mathrm{~s}^{-1}$ and a high-velocity component that extends up to $1300 \mathrm{~km} \mathrm{~s}^{-1}$ (right panel of Fig. 20). This component consists of most of the neutron stars that belong to the high-velocity, high-acceleration group at one second. Both components are similar in strength, but this may depend on the choices of parameters for the considered set of models. The basic result of a bimodality, however, turned out to be very robust against variations in how the extrapolation is done. 
Although the presence or absence of a pronounced $l=1$ mode in the ejecta distribution offers a natural, as well as suggestive, way to obtain a bimodality in the context of our hydrodynamic kick mechanism, we refrain from claiming that our result strongly supports the existence of such a bimodality in the observed distribution of pulsar velocities. There are too many uncertainties that might lead to a filling of the minimum of our distribution. Not only do we assume that our extrapolation law (Eq. (8)) can be applied with the same value for the duration of the average acceleration to all models of our sample, we also consider only a very constrained selection of progenitor models, which is not representative of the true distribution of supernova progenitors. Though our simulations do not reveal systematic differences of the kicks in dependence of the explosion energy or progenitor structure, we do not feel able to exclude that a correlation of both over the range of supernova progenitors could conspire such that the bimodality of Fig. 20 gets wiped out.

The proposed hydrodynamic kick mechanism, however, leads to an unambiguous prediction, which might be tested by future detailed observations of supernova remnants: the measured neutron star velocity should be directed against the momentum of the gaseous supernova ejecta. This is different from many theories that explain pulsar kicks by anisotropic neutrino emission from the nascent neutron star. In that case the direction of the acceleration can be independent of ejecta asymmetries.

Apart from all the assumptions and approximations entering this work and discussed in detail above, the biggest deficiency in the present analysis is that it is based on simulations that assume axial symmetry with the polar axis being a coordinate singularity that is impenetrable for the fluid flow. Currently it is neither clear to what degree pronounced $l=1$ modes of the ejecta distribution and long-lasting downflows of matter to the neutron star can develop in the 3D environment, and how common they are, although first 3D simulations with the setup and input physics described here are promising (they will be presented in a future publication, but see Janka et al. 2005b for some results). Nor is it clear what the distribution of neutron star recoil velocities from 3D models will be. The large number of long-time simulations required by the stochastic nature and long duration of the proposed hydrodynamic kick mechanism, is currently out of reach due to its enormous demand in computer time. Our results must therefore be considered as indicative, but they are far from providing definitive answers.

Acknowledgements. We are grateful to T. Plewa for his contributions to the early stages of this project and his continued interest in this work, to R. Buras, M. Rampp, and S. Bruenn for providing us with post-bounce models, to M. Limongi and S. Woosley for their progenitor models, and to J. Niemeyer for valuable suggestions. We would especially like to thank the anonymous referee for careful reading of the manuscript and many insightful comments and suggestions, which helped us to improve our paper. Support by the Sonderforschungsbereich 375 on "Astroparticle Physics" of the Deutsche Forschungsgemeinschaft is acknowledged. We are also grateful to the Institute for Nuclear Theory at the University of Washington for its hospitality during several visits throughout the duration of this work. The computations were performed on the NEC SX-5/3C of the Rechenzentrum Garching (RZG) and the IBM p690 clusters of the RZG and the John-von-Neumann Institute for Computing in Jülich.

\section{References}

Arras, P., \& Lai, D. 1999a, ApJ, 519, 745

Arras, P., \& Lai, D. 1999b, Phys. Rev. D, 60, 043001

Arzoumanian, Z., Chernoff, D. F., \& Cordes, J. M. 2002, ApJ, 568, 289

Blondin, J. M., \& Mezzacappa, A. 2006, ApJ, 642, 401

Blondin, J. M., Mezzacappa, A., \& DeMarino, C. 2003, ApJ, 584, 971
Brisken, W. F., Fruchter, A. S., Goss, W. M., Herrnstein, R. M., \& Thorsett, S. E. 2003, AJ, 126, 3090

Bruenn, S. W. 1985, ApJS, 58, 771

Bruenn, S. W. 1993, in Nuclear Physics in the Universe, ed. M. W. Guidry, \& M. R. Strayer (Bristol: IOP), 31

Buras, R., Rampp, M., Janka, H.-T., \& Kifonidis, K. 2003, Phys. Rev. Lett., 90, 241101

Buras, R., Rampp, M., Janka, H.-T., \& Kifonidis, K. 2006a, A\&A, 447, 1049

Buras, R., Rampp, M., Janka, H.-T., \& Kifonidis, K. 2006b, A\&A, submitted [arXiv: astro-ph/0512189]

Burrows, A., \& Fryxell, B. A. 1993, ApJ, 418, L33

Burrows, A., \& Hayes, J. 1996, Phys. Rev. Lett., 76, 352

Burrows, A., \& Thompson, T. A. 2004, in Stellar Collapse, ed. C. Fryer (Dordrecht: Kluwer Academic Publishers), 133

Burrows, A., \& Woosley, S. E. 1986, ApJ, 308, 680

Burrows, A., Hayes, J., \& Fryxell, B. A. 1995, ApJ, 450, 830

Burrows, A., Livne, E., Dessart, L., Ott, C. D., \& Murphy, J. 2006, ApJ, 640, 878

Cen, R. 1998, ApJ, 507, L131

Chandrasekhar, S. 1961, Hydrodynamic and hydromagnetic stability (New York: Dover)

Chatterjee, S., Vlemmings, W. H. T., Brisken, W. F., et al. 2005, ApJ, 630, L61

Chugai, N. N. 1984, Soviet Astron. Lett., 10, 87

Colella, P., \& Woodward, P. R. 1984, J. Comput. Phys., 54, 174

Cordes, J. M., \& Chernoff, D. F. 1998, ApJ, 505, 315

Cordes, J. M., Romani, R. W., \& Lundgren, S. C. 1993, Nature, 362, 133

Dorofeev, O. F., Rodionov, V. N., \& Ternov, I. M. 1985, Soviet Astronomy Letters, 11, 123

Einfeldt, B. 1988, SIAM J. Num. Anal., 25, 294

Foglizzo, T. 2001, A\&A, 368, 311

Foglizzo, T. 2002, A\&A, 392, 353

Foglizzo, T., \& Tagger, M. 2000, A\&A, 363, 174

Foglizzo, T., Scheck, L., \& Janka, H.-T. 2006, ApJ, submitted

[arXiv:astro-ph/0507636]

Freedman, D. Z., Schramm, D. N., \& Tubbs, D. L. 1977, Ann. Rev. Nucl. Sci., 27,167

Fryer, C., Burrows, A., \& Benz, W. 1998, ApJ, 496, 333

Fryer, C. L. 2004, ApJ, 601, L175

Fryer, C. L., \& Kusenko, A. 2006, ApJS, 163, 335

Fryer, C. L., \& Warren, M. S. 2002, ApJ, 574, L65

Fryer, C. L., \& Warren, M. S. 2004, ApJ, 601, 391

Fuller, G. M., Kusenko, A., Mocioiu, I., \& Pascoli, S. 2003, Phys. Rev. D, 68, 103002

Galletti, P. \& Foglizzo, T. 2005, in Proc. of the SF2A meeting, 17-30 June 2005, Strasbourg, EDPS Conf. Ser. in Astronomy \& Astrophysics, ed. F. Casoli, T. Contini, H. M. Hameury, \& L. Pagani [arXiv: astro-ph/0509635]

Goldreich, P., Lai, D., \& Sahrling, M. 1996, in Unsolved Problems in Astrophysics, ed. J. N. Bahcall, \& J. P. Ostriker (Princeton: Princeton Univ. Press)

Hansen, B. M. S., \& Phinney, E. S. 1997, MNRAS, 291, 569

Heger, A., Woosley, S., Langer, N., \& Spruit, H. C. 2004, in Proc. IAU Symp. 215, ed. A. Maeder, \& P. Eenens (San Francisco: ASP), 591 [arXiv:astro-ph/0301374]

Herant, M. 1995, Phys. Rep., 256, 117

Herant, M., Benz, W., \& Colgate, S. 1992, ApJ, 395, 642

Herant, M., Benz, W., Hix, W. R., Fryer, C. L., \& Colgate, S. A. 1994, ApJ, 435, 339

Hobbs, G., Lorimer, D. R., Lyne, A. G., \& Kramer, M. 2005, MNRAS, 360, 974

James, F. 1994, Comput. Phys. Commun., 79, 111

James, F. 1996, Comput. Phys. Commun., 97, 357

Janka, H.-T. 1990, in Procs. of the 5th Workshop on Nuclear Astrophysics, held 30 January-4 February 1989 at Ringberg Castle, Tegernsee, FRG, ed. W. Hillebrandt, \& E. Müller (Garching: Max-Planck-Institut für Astrophysik), 130

Janka, H.-T. 1991a, A\&A, 244, 378

Janka, H.-T. 1991b, Dissertation, Technische Universität München

Janka, H.-T., \& Hillebrandt, W. 1989, A\&AS, 78, 375

Janka, H.-T., \& Müller, E. 1994, A\&A, 290, 496

Janka, H.-T., \& Müller, E. 1996, A\&A, 306, 167

Janka, H.-T., Buras, R., Kifonidis, K., Plewa, T., \& Rampp, M. 2003, in From Twilight to Highlight: The Physics of Supernovae, Proc. of the ESO/MPA/MPE Workshop, Garching, Germany, July 29-31, 2002, ed. W. Hillebrandt, \& B. Leibundgut (Berlin: Springer), 39 [arXiv: astro-ph/0212316]

Janka, H.-T., Buras, R., Kifonidis, K., Plewa, T., \& Rampp, M. 2004a, in Stellar Collapse, ed. C. Fryer (Dordrecht: Kluwer Academic Publishers), 65 [arXiv: astro-ph/0212314] 
Janka, H.-T., Buras, R., Kitaura Joyanes, F., Marek, A. \& Rampp, M. 2004b in Procs. of the 12th Workshop on Nuclear Astrophysics, Ringberg Castle, Tegernsee, March 22-27, 2004, Report MPA-P14, Max-Planck-Institut für Astrophysik, Garching, ed. E. Müller, \& H.-T. Janka, 150 [arXiv: astro-ph/0405289]

Janka, H.-T., Kifonidis, K., Buras, R., Marek, A., \& Rampp, M. 2005a, in Cosmic Explosions (On the 10th Anniversary of SN1993J), Proceedings IAU Coll. 192, Valencia, Spain, April 22-26, 2003, ed. J. Marcaide, \& K. Weiler (Berlin: Springer Verlag), 253 [arXiv: astro-ph/0401461]

Janka, H.-T., Scheck, L., Kifonidis, K., Müller, E., \& Plewa, T. 2005b, in The Fate of the Most Massive Stars, ed. R. Humphreys \& K. Stanek (San Francisco: ASP), ASP Conf. Ser., 332, 372 [arXiv: astro-ph/0408439]

Johnston, S., Hobbs, G., Vigeland, S., et al. 2005, MNRAS, 364, 1397

Keil, M. T., Raffelt, G. G., \& Janka, H.-T. 2003, ApJ, 590, 971

Khokhlov, A. M., Höflich, P. A., Oran, E. S., et al. 1999, ApJ, 524, L107

Kifonidis, K., Plewa, T., Janka, H.-T., \& Müller, E. 2003, A\&A, 408, 621

Kifonidis, K., Plewa, T., Scheck, L., Janka, H.-T., \& Müller, E. 2006, A\&A, 453, 661

Kotake, K., Yamada, S., \& Sato, K. 2003, ApJ, 595, 304

Kotake, K., Sawai, H., Yamada, S., \& Sato, K. 2004, ApJ, 608, 391

Kuhlen, M., Woosley, W. E., \& Glatzmaier, G. A. 2003, in 3D Stellar Evolution, ed. S. Turcotte, S. Keller, \& R. Cavallo (San Francisco: ASP), ASP Conf. Ser., 293, 147

Lai, D. 2001, in Physics of Neutron Star Interiors, ed. D. Blaschke, N. Glendenning, \& A. Sedrakian (Berlin: Springer), LNP, 578, 424

Lai, D., \& Goldreich, P. 2000, ApJ, 535, 402

Lai, D., Chernoff, D. F. \& Cordes, J. M. 2001, ApJ, 549, 1111

Lattimer, J. M., \& Swesty, F. D. 1991, Nucl. Phys. A, 535, 331

Leonard, D. C., Filippenko, A. V., Ganeshalingam, M., et al. 2006, Nature, 440, 505

Liebendörfer, M., Mezzacappa, A., Thielemann, F., et al. 2001, Phys. Rev. D, 63, 103004

Liebendörfer, M., Rampp, M., Janka, H.-T., \& Mezzacappa, A. 2005, ApJ, 620, 840

Limongi, M., Straniero, O., \& Chieffi, A. 2000, ApJS, 129, 625

Livne, E., Burrows, A., Walder, R., Lichtenstadt, I., \& Thompson, T. A. 2004, ApJ, 609, 277

Lüscher, M. 1994, Comput. Phys. Commun., 79, 100

Lyne, A. G., \& Lorimer, D. R. 1994, Nature, 369, 127
Marek, A., Dimmelmeier, H., Janka, H.-T., Müller, E., \& Buras, R. 2006, A\&A, 445,273

Minerbo, G. N. 1978, J. Quant. Spectrosc. Radiat. Transfer, 20, 541

Müller, E., \& Steinmetz, M. 1995, Comput. Phys. Commun., 89, 45

Müller, E., Rampp, M., Buras, R., Janka, H.-T., \& Shoemaker, D. H. 2004, ApJ, 603, 221

Murphy, J. W., Burrows, A., \& Heger, A. 2004, ApJ, 615, 460

Myra, E. S., \& Burrows, A. 1990, ApJ, 364, 222

Ohnishi, N., Kotake, K., \& Yamada, S. 2006, ApJ, 641, 1018

Rampp, M., \& Janka, H.-T. 2000, ApJ, 539, L33

Rampp, M., \& Janka, H.-T. 2002, A\&A, 396, 361

Scheck, L., Plewa, T., Janka, H.-T., Kifonidis, K., \& Müller, E. 2004, Phys. Rev. Lett., 92, 011103

Schinder, P. J., Schramm, D. N., Wiita, P. J., Margolis, S. H., \& Tubbs, D. L. 1987, ApJ, 313, 531

Sehgal, I. 1974, Nucl. Phys. B, 70, 61

Socrates, A., Blaes, O., Hungerford, A., \& Fryer, C. L. 2005, ApJ, 632, 531

Straumann, N. 1989, in Particle Physics and Astrophysics, ed. H. Mitter, \& F. Widder (Berlin: Springer-Verlag), 79

Tassoul, J.-L. 1978, Theory of Rotating Stars (Princeton, New Jersey: Princeton University Press)

Thompson, C. 2000, ApJ, 534, 915

Thompson, T. A., Burrows, A., \& Meyer, B. S. 2001, ApJ, 562, 887

Thompson, T. A., Burrows, A., \& Pinto, P. A. 2003, ApJ, 592, 434

Tubbs, D. L. 1979, ApJ, 231, 846

Tubbs, D. L. \& Schramm, D. N. 1975, ApJ, 201, 467

Wang, C., Lai, D., \& Han, J. L. 2006, ApJ, 639, 1007

Wang, L., Howell, D. A., Höflich, P., \& Wheeler, J. C. 2001, ApJ, 550, 1030

Wang, L., Wheeler, J. C., Höflich, P., et al. 2002, ApJ, 579, 671

Wang, L., Baade, D., Höflich, P., \& Wheeler, J. C. 2003, ApJ, 592, 457

Wheeler, J. C., Meier, D. L., \& Wilson, J. R. 2002, ApJ, 568, 807

Woodward, P. R., Porter, D. H., \& Jacobs, M. 2003, in 3D Stellar Evolution, ed. S. Turcotte, S. Keller, \& R. Cavallo (San Francisco: Astron. Society of the Pacific), ASP Conf. Ser., 293, 45

Woosley, S. E. 1987, in The Origin and Evolution of Neutron Stars, ed. D. J. Helfand, \& J.-H. Huang, IAU Symp. 125, 255

Woosley, S. E., \& Weaver, T. A. 1995, ApJS, 101, 181

Woosley, S. E., Pinto, P. A., \& Ensman, L. 1988, ApJ, 324, 466

Zou, W. Z., Hobbs, G., Wang, N., et al. 2005, MNRAS, 362, 1189 


\section{Online Material}




\section{Appendix A: Post-processing of the simulations}

In the following we define and tabulate some interesting characteristic quantities that were evaluated for our about 80 hydrodynamic models by post-processing the data of the simulations. To keep the evaluation as straightforward as possible we sometimes employ approximations which we will detail below.

The inner boundary condition for the neutrinos is constrained by the parameters $t_{L}, \Delta E_{v, \text { core }}^{\text {tot }}(t)$ and $\Delta Y_{\mathrm{e} \text {,core }}(t)$. Their definitions are given in Appendix D.2, while their actual values (at $t=1 \mathrm{~s}$ in case of the time-dependent quantities) are listed in Tables A.1-A.5. A characterising value for the neutrino luminosities imposed at this inner boundary is

$L_{\mathrm{ib}}(t) \equiv L_{\mathrm{e}, v_{\mathrm{e}}}\left(R_{\mathrm{ib}}, t\right)+L_{\mathrm{e}, \bar{v}_{\mathrm{e}}}\left(R_{\mathrm{ib}}, t\right)$,

where $L_{\mathrm{e}, v_{\mathrm{e}}}$ and $L_{\mathrm{e}, \bar{v}_{\mathrm{e}}}$ are the energy luminosities of $v_{\mathrm{e}}$ and $\bar{v}_{\mathrm{e}}$ defined in Appendix D.1. Equation (A.1) neglects the contribution from heavy-lepton neutrinos, whose interactions in the computational domain are less important than those of $v_{\mathrm{e}}$ and $\bar{v}_{\mathrm{e}}$, and who, in particular, do not contribute to the neutrino heating behind the shock at a significant level.

We also consider the sum of the $v_{\mathrm{e}}$ and $\bar{v}_{\mathrm{e}}$ luminosities at a radius of $500 \mathrm{~km}$,

$L_{500}(t)=L_{\mathrm{e}, v_{\mathrm{e}}}(r=500 \mathrm{~km}, t)+L_{\mathrm{e}, \bar{v}_{\mathrm{e}}}(r=500 \mathrm{~km}, t)$,

and define the time average of this quantity in the time interval $\left[0, t_{\exp }\right]$ as

$\left\langle L_{500}\right\rangle=t_{\exp }^{-1} \int_{0}^{t_{\text {exp }}} L_{500}(t) \mathrm{d} t$.

The value of $\left\langle L_{500}\right\rangle$ represents approximatively the $\left(v_{\mathrm{e}}+\bar{v}_{\mathrm{e}}\right)$ luminosity that is responsible for the energy deposition behind the supernova shock until the explosion sets in at a post-bounce time $t=t_{\text {exp }}$. Therefore the difference between $\left\langle L_{500}\right\rangle$ and $L_{\mathrm{ib}}$ can be considered as a rough measure for the radial change of the neutrino luminosities in contrast to their constancy in case of the light-bulb scheme used by Janka \& Müller (1996). Furthermore, we list the total energy in $v_{\mathrm{e}}$ and $\bar{v}_{\mathrm{e}}$ neutrinos that streams through a sphere with a radius of $500 \mathrm{~km}$ in the time interval $[0, t]$,

$\Delta E_{500}(t)=\int_{0}^{t}\left(L_{\mathrm{e}, v_{\mathrm{e}}}+L_{\mathrm{e}, \bar{v}_{\mathrm{e}}}\right)\left(r=500 \mathrm{~km}, t^{\prime}\right) \mathrm{d} t^{\prime}$.

The explosion energy, $E_{\text {exp }}$, of a model is defined as the sum of the total energy of all zones of the grid where this energy is positive, i.e.

$E_{\exp }=\sum_{e_{\mathrm{tot}, i}>0} e_{\mathrm{tot}, i} \Delta m_{i}$

where $i$ is the zone counter, $\Delta m_{i}$ the mass contained in zone $i$, and the total specific energy $e_{\text {tot }}$ is given by the sum of the specific gravitational, kinetic, and internal energies,

$e_{\mathrm{tot}}=e_{\mathrm{grav}}+\frac{1}{2} v^{2}+e_{\mathrm{int}}$.

For the sake of simplicity we use here the 1D Newtonian expression

$e_{\text {grav }}(r)=-\frac{G M(r)}{r}$

to evaluate the gravitational energy, neglecting the relatively small general relativistic corrections, which have been taken into account in the simulations.
The explosion time scale, $t_{\text {exp }}$, is defined as the time after the start of the simulation when $E_{\exp }$ exceeds $10^{48} \mathrm{erg}$. It turns out that the exact choice of this threshold value does not matter very much. Other definitions of the explosion time scale (e.g., linked to the time when the expansion velocity of the shock exceeds a certain value) do also not lead to qualitatively different results.

To characterise the deviation of the shape of the supernova shock from a sphere we introduce a shock deformation parameter,

$d_{\text {shock }}:=\frac{\max \left(R_{\mathrm{s}}(\theta) \cos \theta\right)-\min \left(R_{\mathrm{S}}(\theta) \cos (\theta)\right)}{2 \times \max \left(R_{\mathrm{S}}(\theta) \sin \theta\right)}-1$,

where $R_{\mathrm{S}}(\theta)$ is the local shock radius as a function of polar angle $\theta$. The numerator and denominator in Eq. (A.8) are the maximum shock diameters in projection on the symmetry axis and perpendicular to it, respectively. A prolate deformation leads to a positive value of $d_{\text {shock }}$, an oblate deformation gives a negative value. Note that a linear shift of the shock surface in the direction of the $z$-axis does not change $d_{\text {shock}}$.

The neutron star mass and the neutron star radius are considered to be associated with a certain value of the density, $\rho_{\mathrm{ns}}=10^{11} \mathrm{~g} \mathrm{~cm}^{-3}$. The neutron star radius, $R_{\mathrm{ns}}$, is then simply defined as the radius where the lateral average of the density is equal to $\rho_{\mathrm{ns}}$, and the baryonic mass of the neutron star, $M_{\mathrm{ns}}$, is given by the sum of the central point mass and the mass integral over all grid zones with densities $\geq \rho_{\mathrm{ns}}$.

In evaluating the neutron star recoil velocity, $\boldsymbol{v}_{\mathrm{ns}}$, we have to distinguish between simulations in which we consider the neutron star motion relative to the ejecta by changing the frame of reference after each time step (see Sect. 2.3 and Appendix B), and simulations in which this motion is not accounted for. In the first case no post-processing is required, because the neutron star velocity is given at all times by the accumulated effects of the Galilei transformations applied until time $t$ or time step $m$,

$\boldsymbol{v}_{\mathrm{ns}}(t)=\sum_{n=1, \ldots, m} \Delta \boldsymbol{v}_{\mathrm{core}}^{n}$

where $\Delta \boldsymbol{v}_{\text {core }}^{n}$ is given by Eq. (B.9). In the second case, $\boldsymbol{v}_{\mathrm{ns}}$ is computed a posteriori, by making use of linear momentum conservation. The total momentum of the system, i.e. the sum of the neutron star momentum $\boldsymbol{P}_{\mathrm{ns}}=M_{\mathrm{ns}} \boldsymbol{v}_{\mathrm{ns}}$ and the momentum of the surrounding gas on the computational grid, $\boldsymbol{P}_{\text {gas }}$, is initially zero (because all models that we consider are spherically symmetric or equatorially and axially symmetric just after collapse). Hence we have for all times

$\boldsymbol{v}_{\mathrm{ns}}(t)=-\boldsymbol{P}_{\mathrm{gas}}(t) / M_{\mathrm{ns}}(t)$,

and $v_{\mathrm{ns}}(t)$ can be determined by evaluating the neutron star mass and the momentum integral of the ejecta gas,

$\boldsymbol{P}_{\text {gas }}(t)=\int_{R_{\mathrm{ns}}<r<\infty} \rho \boldsymbol{v} \mathrm{d} V$

Here $\mathrm{d} V=r^{2} \sin \theta \mathrm{d} r \mathrm{~d} \theta \mathrm{d} \phi$. Note that the volume integral in Eq. (A.11) is limited by the outer boundary of our Eulerian grid and that the momentum flux associated with anisotropic mass flow over the grid boundary would have to be taken into account.

Equation (A.10) may actually also be coined in terms of an anisotropy parameter of the ejecta, $\alpha_{\text {gas }}$ (see Janka \& Müller 1994; Herant 1995). To accomplish this, we make use of the following scalar quantity

$P_{\mathrm{ej}}(t):=\int_{R_{\mathrm{ns}}}^{R_{\mathrm{s}}(\theta)} \rho|\boldsymbol{v}| \mathrm{d} V$ 
Table A.1. Simulations based on the Woosley et al. (1988)/Bruenn (1993) post-bounce model. The luminosity time scale $t_{L}$ is $1 \mathrm{~s}$. Unless noted otherwise the inertial mass of the neutron star is assumed to be infinite for these and the simulations listed in the following tables, i.e. the neutron star takes up momentum but cannot move on the grid. For the definitions of the listed quantities see the main text. All time-dependent quantities are given at a time $t=1 \mathrm{~s}$, when we terminated the simulations. Energies are given in units of $1 \mathrm{~B}=1 \mathrm{Bethe}=10^{51} \mathrm{erg}$.

\begin{tabular}{|c|c|c|c|c|c|c|c|c|c|c|c|c|c|}
\hline Model & $\begin{array}{r}L_{\mathrm{ib}} \\
{[\mathrm{B} / \mathrm{s}]}\end{array}$ & $\begin{array}{r}\Delta E_{\nu, \text { core }}^{\text {tot }} \\
{[\mathrm{B}]}\end{array}$ & $\Delta Y_{\mathrm{e}, \mathrm{core}}$ & $\begin{array}{r}\left\langle L_{500}\right\rangle \\
{[\mathrm{B} / \mathrm{s}]}\end{array}$ & $\begin{array}{r}\Delta E_{500} \\
{[\mathrm{~B}]}\end{array}$ & $\begin{array}{r}E_{\exp } \\
{[\mathrm{B}]}\end{array}$ & $\begin{array}{c}t_{\exp } \\
{[\mathrm{s}]}\end{array}$ & $\begin{array}{r}M_{\mathrm{ns}} \\
{\left[M_{\odot}\right]}\end{array}$ & $\begin{array}{c}v_{z}^{\mathrm{ns}} \\
{\left[\mathrm{km} \mathrm{s}^{-1}\right]}\end{array}$ & $\begin{array}{r}v_{z}^{\mathrm{ns}, v} \\
{\left[\mathrm{~km} \mathrm{~s}^{-1}\right]}\end{array}$ & $\begin{array}{r}a_{z}^{\mathrm{ns}} \\
{\left[\mathrm{km} \mathrm{s}^{-2}\right]}\end{array}$ & $\alpha_{\text {gas }}$ & $d_{\text {shock }}$ \\
\hline$\overline{\mathrm{B} 10}$ & 24.7 & 59.6 & 0.09 & 57.1 & 45.9 & 0.19 & 0.294 & 1.426 & -164.1 & 44.4 & -180.2 & 0.24 & 0.67 \\
\hline B11 & 27.2 & 65.5 & 0.10 & 58.8 & 46.3 & 0.27 & 0.280 & 1.401 & -23.6 & 0.7 & -248.9 & 0.03 & 0.97 \\
\hline B12 & 29.7 & 71.5 & 0.11 & 60.6 & 48.7 & 0.37 & 0.220 & 1.399 & -389.5 & 45.0 & -372.4 & 0.32 & 0.06 \\
\hline B12-1 & 29.7 & 71.5 & 0.11 & 60.5 & 47.5 & 0.33 & 0.228 & 1.377 & 72.8 & -4.7 & 47.9 & 0.07 & 0.22 \\
\hline B12-2 & 29.7 & 71.5 & 0.11 & 60.9 & 48.5 & 0.39 & 0.212 & 1.391 & 85.8 & 9.7 & 345.7 & 0.07 & 0.82 \\
\hline B12-3 & 29.7 & 71.5 & 0.11 & 60.9 & 46.5 & 0.38 & 0.207 & 1.369 & 242.0 & 2.0 & 464.3 & 0.18 & 0.97 \\
\hline B12-4 & 29.7 & 71.5 & 0.11 & 61.1 & 47.7 & 0.35 & 0.216 & 1.385 & -115.1 & 20.4 & -154.2 & 0.10 & 0.51 \\
\hline B12-5 & 29.7 & 71.5 & 0.11 & 61.0 & 47.8 & 0.33 & 0.211 & 1.387 & -206.9 & 11.6 & -483.1 & 0.19 & 0.52 \\
\hline B13 & 32.2 & 77.5 & 0.12 & 62.4 & 49.6 & 0.45 & 0.188 & 1.378 & -355.3 & 32.0 & -408.0 & 0.25 & 0.36 \\
\hline B14 & 34.6 & 83.4 & 0.13 & 63.6 & 49.6 & 0.51 & 0.198 & 1.345 & -128.0 & -11.2 & -66.7 & 0.07 & 0.40 \\
\hline B15 & 37.1 & 89.4 & 0.14 & 65.3 & 50.3 & 0.65 & 0.162 & 1.318 & 36.1 & -1.0 & 36.0 & 0.02 & 0.27 \\
\hline B16 & 39.6 & 95.3 & 0.15 & 66.3 & 51.8 & 0.81 & 0.160 & 1.305 & -214.6 & -2.6 & -334.4 & 0.08 & 0.57 \\
\hline B17 & 42.1 & 101.3 & 0.15 & 67.6 & 53.3 & 0.95 & 0.146 & 1.289 & -25.5 & 14.8 & -102.6 & 0.01 & 0.05 \\
\hline B17-1 & 42.1 & 101.3 & 0.15 & 67.8 & 53.4 & 0.92 & 0.160 & 1.290 & -354.0 & 5.6 & -202.2 & 0.12 & 0.31 \\
\hline B18 & 44.5 & 107.3 & 0.16 & 68.3 & 54.8 & 1.16 & 0.152 & 1.275 & 515.3 & 5.2 & 290.5 & 0.15 & 0.42 \\
\hline B18-1 & 44.5 & 107.3 & 0.16 & 68.4 & 54.7 & 1.12 & 0.154 & 1.274 & -126.5 & -0.8 & -49.1 & 0.04 & 0.20 \\
\hline B18-2 & 44.5 & 107.3 & 0.16 & 68.9 & 54.7 & 1.14 & 0.152 & 1.268 & 82.5 & -5.2 & 16.5 & 0.02 & 0.07 \\
\hline B18-3 & 44.5 & 107.3 & 0.16 & 68.8 & 57.1 & 1.15 & 0.142 & 1.305 & 798.8 & -41.2 & 552.1 & 0.24 & -0.06 \\
\hline B18-4 & 44.5 & 107.3 & 0.16 & 68.2 & 54.6 & 1.14 & 0.150 & 1.272 & -171.6 & 4.0 & 65.7 & 0.05 & 0.46 \\
\hline B18-5 & 44.5 & 107.3 & 0.16 & 68.5 & 55.2 & 1.09 & 0.164 & 1.280 & -121.8 & -0.9 & 15.4 & 0.04 & -0.02 \\
\hline B18-6 & 44.5 & 107.3 & 0.16 & 68.7 & 55.4 & 1.11 & 0.160 & 1.283 & 502.1 & -20.6 & 220.0 & 0.15 & -0.06 \\
\hline B18-g1 & 44.5 & 107.3 & 0.16 & 68.7 & 54.5 & 1.12 & 0.142 & 1.269 & -60.3 & 3.9 & -55.4 & 0.02 & 0.06 \\
\hline B18-g2 & 44.5 & 107.3 & 0.16 & 68.7 & 54.8 & 1.12 & 0.138 & 1.273 & 267.9 & -8.1 & 126.7 & 0.08 & 0.28 \\
\hline B18-g3 & 44.5 & 107.3 & 0.16 & 68.5 & 54.9 & 1.10 & 0.150 & 1.274 & -7.4 & -3.5 & 0.9 & 0.00 & 0.02 \\
\hline B18-g4 & 44.5 & 107.3 & 0.16 & 68.7 & 54.5 & 1.16 & 0.132 & 1.270 & -416.8 & 1.7 & -150.9 & 0.11 & 0.37 \\
\hline B19-g1 & 47.0 & 113.2 & 0.17 & 69.6 & 55.9 & 1.31 & 0.148 & 1.253 & -273.8 & 0.3 & -96.7 & 0.07 & 0.41 \\
\hline B19-g2 & 47.0 & 113.2 & 0.17 & 69.5 & 56.0 & 1.33 & 0.148 & 1.255 & 188.5 & 6.4 & 48.8 & 0.05 & 0.15 \\
\hline B19-g3 & 47.0 & 113.2 & 0.17 & 70.0 & 56.6 & 1.26 & 0.132 & 1.263 & 366.6 & 1.1 & 183.7 & 0.10 & 0.13 \\
\hline B19-g4 & 47.0 & 113.2 & 0.17 & 70.0 & 56.8 & 1.33 & 0.130 & 1.267 & 477.1 & -18.3 & 195.6 & 0.12 & -0.02 \\
\hline B20 & 49.5 & 119.2 & 0.18 & 71.0 & 57.3 & 1.49 & 0.128 & 1.238 & 133.2 & 5.6 & 52.6 & 0.03 & 0.40 \\
\hline B21 & 51.9 & 125.1 & 0.19 & 72.1 & 58.5 & 1.72 & 0.122 & 1.222 & 30.6 & -0.9 & -20.2 & 0.01 & 0.24 \\
\hline
\end{tabular}

Table A.2. Simulations based on the Limongi et al. (2000)/Rampp post-bounce model. The luminosity time scale $t_{L}$ is $0.7 \mathrm{~s}$ for these simulations. For more details, see the caption of Table A.1.

\begin{tabular}{lrrrrrrrrrrrrr}
\hline \hline Model & $\begin{array}{r}L_{\mathrm{ib}} \\
{[\mathrm{B} / \mathrm{s}]}\end{array}$ & $\begin{array}{r}\Delta E_{v, \text { core }}^{\text {tot }} \\
{[\mathrm{B}]}\end{array}$ & $\Delta Y_{\mathrm{e}, \text { core }}$ & $\begin{array}{r}\left\langle L_{500}\right\rangle \\
{[\mathrm{B} / \mathrm{s}]}\end{array}$ & $\begin{array}{r}\Delta E_{500} \\
{[\mathrm{~B}]}\end{array}$ & $\begin{array}{r}E_{\text {exp }} \\
{[\mathrm{B}]}\end{array}$ & $\begin{array}{r}t_{\text {exp }} \\
{[\mathrm{s}]}\end{array}$ & $\begin{array}{r}M_{\text {ns }} \\
{\left[M_{\odot}\right]}\end{array}$ & $\begin{array}{r}v_{z}^{\text {ns }} \\
{\left[\mathrm{km} \mathrm{s}^{-1}\right]}\end{array}$ & $\begin{array}{r}v_{z}^{\text {ns, } v} \\
{\left[\mathrm{~km} \mathrm{~s}^{-1}\right]}\end{array}$ & $\begin{array}{c}a_{z}^{\text {ns }} \\
{\left[\mathrm{km} \mathrm{s}^{-2}\right]}\end{array}$ & $\alpha_{\text {gas }}$ & $d_{\text {shock }}$ \\
\hline L12 & 42.4 & 94.6 & 0.13 & 90.7 & 70.7 & 0.51 & 0.321 & 1.677 & 278.5 & -12.9 & 334.3 & 0.24 & 0.11 \\
L13 & 45.9 & 102.5 & 0.14 & 91.7 & 69.2 & 0.68 & 0.268 & 1.620 & -92.6 & -5.9 & -333.6 & 0.05 & 0.77 \\
L14 & 49.5 & 110.4 & 0.15 & 94.6 & 72.8 & 0.81 & 0.280 & 1.628 & 482.1 & -22.0 & 297.1 & 0.26 & 0.31 \\
L15 & 53.0 & 118.3 & 0.17 & 96.2 & 75.2 & 1.02 & 0.266 & 1.617 & -239.5 & -3.9 & -378.5 & 0.10 & 0.63 \\
L16 & 56.5 & 126.2 & 0.18 & 97.8 & 76.3 & 1.07 & 0.256 & 1.586 & -437.9 & 12.8 & -715.2 & 0.17 & 0.47 \\
L17 & 60.1 & 134.0 & 0.19 & 100.3 & 77.4 & 1.19 & 0.256 & 1.558 & -24.7 & 5.5 & -47.6 & 0.01 & 0.37 \\
\hline
\end{tabular}

Table A.3. Simulations based on the non-rotating Woosley \& Weaver (1995)/Buras et al. (2003) post-bounce model. The luminosity time scale $t_{L}$ is $1 \mathrm{~s}$ for these simulations. For more details, see the caption of Table A.1.

\begin{tabular}{|c|c|c|c|c|c|c|c|c|c|c|c|c|c|}
\hline Model & $\begin{array}{r}L_{\mathrm{ib}} \\
{[\mathrm{B} / \mathrm{s}]}\end{array}$ & $\begin{array}{r}\Delta E_{v, \text { core }}^{\text {tot }} \\
{[\mathrm{B}]}\end{array}$ & $\Delta Y_{\mathrm{e}, \text { core }}$ & $\begin{array}{r}\left\langle L_{500}\right\rangle \\
{[\mathrm{B} / \mathrm{s}]}\end{array}$ & $\begin{array}{r}\Delta E_{500} \\
{[\mathrm{~B}]}\end{array}$ & $\begin{array}{r}E_{\text {exp }} \\
{[\mathrm{B}]}\end{array}$ & $\begin{array}{r}t_{\exp } \\
{[\mathrm{s}]}\end{array}$ & $\begin{array}{r}M_{\mathrm{ns}} \\
{\left[M_{\odot}\right]}\end{array}$ & $\begin{array}{r}v_{z}^{\text {ns }} \\
{\left[\mathrm{km} \mathrm{s}^{-1}\right]}\end{array}$ & $\begin{array}{r}v_{z}^{\mathrm{ns}, v} \\
{\left[\mathrm{~km} \mathrm{~s}^{-1}\right]}\end{array}$ & $\begin{array}{r}a_{z}^{\text {ns }} \\
{\left[\mathrm{km} \mathrm{s}^{-2}\right]}\end{array}$ & $\alpha_{\text {gas }}$ & $d_{\text {shock }}$ \\
\hline W10 & 24.7 & 59.6 & 0.09 & 64.3 & 55.4 & 0.21 & 0.420 & 1.568 & -129.8 & 42.1 & -443.1 & 0.15 & 0.81 \\
\hline W12 & 29.7 & 71.5 & 0.11 & 69.0 & 53.9 & 0.31 & 0.322 & 1.501 & -97.7 & -9.7 & -132.5 & 0.10 & 0.61 \\
\hline W12-1 & 29.7 & 71.5 & 0.11 & 68.0 & 59.5 & 0.32 & 0.374 & 1.563 & -363.8 & 81.2 & -377.0 & 0.32 & 0.13 \\
\hline W14 & 34.6 & 83.4 & 0.13 & 72.9 & 56.6 & 0.46 & 0.250 & 1.473 & -62.0 & -1.5 & 66.1 & 0.04 & 0.37 \\
\hline W16 & 39.6 & 95.3 & 0.15 & 76.0 & 58.5 & 0.67 & 0.244 & 1.430 & 287.2 & -5.5 & 464.2 & 0.14 & 0.68 \\
\hline W18 & 44.5 & 107.3 & 0.16 & 79.3 & 61.5 & 0.89 & 0.226 & 1.401 & -283.6 & 4.2 & -290.1 & 0.11 & 0.44 \\
\hline W20 & 49.5 & 119.2 & 0.18 & 82.0 & 63.5 & 1.36 & 0.216 & 1.354 & -377.3 & 0.6 & -277.0 & 0.10 & 0.39 \\
\hline
\end{tabular}


Table A.4. Simulations based on the rotating Woosley \& Weaver (1995)/Buras et al. (2003) post-bounce model. The luminosity time scale $t_{L}$ is $1 \mathrm{~s}$. For more details, see the caption of Table A.1.

\begin{tabular}{|c|c|c|c|c|c|c|c|c|c|c|c|c|c|}
\hline Model & $\begin{array}{r}L_{\mathrm{ib}} \\
{[\mathrm{B} / \mathrm{s}]} \\
\end{array}$ & $\begin{array}{r}\Delta E_{v, \text { core }}^{\text {tot }} \\
{[\mathrm{B}]} \\
\end{array}$ & $\Delta Y_{\mathrm{e}, \text { core }}$ & $\begin{array}{r}\left\langle L_{500}\right\rangle \\
{[\mathrm{B} / \mathrm{s}]}\end{array}$ & $\begin{array}{r}\Delta E_{500} \\
{[\mathrm{~B}]} \\
\end{array}$ & $\begin{array}{r}E_{\exp } \\
{[\mathrm{B}]}\end{array}$ & $\begin{array}{r}t_{\exp } \\
{[\mathrm{s}]}\end{array}$ & $\begin{array}{r}M_{\mathrm{ns}} \\
{\left[M_{\odot}\right]}\end{array}$ & $\begin{array}{r}v_{z}^{\mathrm{ns}} \\
{\left[\mathrm{km} \mathrm{s}^{-1}\right]}\end{array}$ & $\begin{array}{r}v_{z}^{\mathrm{ns}, v} \\
{\left[\mathrm{~km} \mathrm{~s}^{-1}\right]}\end{array}$ & $\begin{array}{r}a_{z}^{\mathrm{ns}} \\
{\left[\mathrm{km} \mathrm{s}^{-2}\right]}\end{array}$ & $\alpha_{\text {gas }}$ & $d_{\text {shock }}$ \\
\hline R10 & 24.7 & 59.6 & 0.09 & 59.9 & 48.8 & 0.25 & 0.418 & 1.521 & -15.4 & -14.3 & -118.7 & 0.02 & -0.02 \\
\hline R12 & 29.7 & 71.5 & 0.11 & 64.6 & 49.9 & 0.50 & 0.316 & 1.461 & -235.8 & 17.5 & -203.4 & 0.16 & 0.15 \\
\hline R14 & 34.6 & 83.4 & 0.13 & 69.2 & 52.4 & 0.69 & 0.264 & 1.420 & 88.4 & 14.6 & 86.9 & 0.04 & 0.15 \\
\hline R16 & 39.6 & 95.3 & 0.14 & 71.9 & 56.0 & 0.98 & 0.256 & 1.396 & 321.2 & -8.9 & 210.1 & 0.11 & 0.06 \\
\hline R18 & 44.5 & 107.3 & 0.16 & 75.8 & 58.3 & 1.24 & 0.232 & 1.349 & -4.8 & -3.7 & -26.7 & 0.00 & -0.07 \\
\hline R18-g & 44.5 & 107.3 & 0.16 & 75.8 & 58.5 & 1.23 & 0.226 & 1.352 & -113.9 & 2.1 & -188.1 & 0.03 & 0.07 \\
\hline R20 & 49.5 & 119.2 & 0.18 & 78.8 & 60.9 & 1.64 & 0.214 & 1.309 & 280.1 & 0.8 & 123.9 & 0.06 & 0.14 \\
\hline
\end{tabular}

Table A.5. Simulations based on the Woosley et al. (1988)/Bruenn (1993) post-bounce model. The luminosity time scale $t_{L}$ is $1 \mathrm{~s}$. For more details, see the caption of Table A.1. Different from the models listed in all other tables, the recoil motion of the neutron star was accounted for in the simulations listed here (as described in Sect. 2.3 and Appendix B).

\begin{tabular}{|c|c|c|c|c|c|c|c|c|c|c|c|c|c|}
\hline Model & $\begin{array}{r}L_{\mathrm{ib}} \\
{[\mathrm{B} / \mathrm{s}]}\end{array}$ & $\begin{array}{r}\Delta E_{v, \mathrm{core}}^{\mathrm{tot}} \\
{[\mathrm{B}]}\end{array}$ & $\Delta Y_{\mathrm{e}, \text { core }}$ & $\begin{array}{r}\left\langle L_{500}\right\rangle \\
{[\mathrm{B} / \mathrm{s}]}\end{array}$ & $\begin{array}{r}\Delta E_{500} \\
{[\mathrm{~B}]}\end{array}$ & $\begin{array}{r}E_{\exp } \\
{[\mathrm{B}]}\end{array}$ & $\begin{array}{l}t_{\exp } \\
{[\mathrm{s}]}\end{array}$ & $\begin{array}{r}M_{\mathrm{ns}} \\
{\left[M_{\odot}\right]}\end{array}$ & $\begin{array}{r}v_{z}^{\mathrm{ns}} \\
{\left[\mathrm{km} \mathrm{s}^{-1}\right]}\end{array}$ & $\begin{array}{r}v_{z}^{\mathrm{ns}, v} \\
{\left[\mathrm{~km} \mathrm{~s}^{-1}\right]}\end{array}$ & $\begin{array}{r}a_{z}^{\mathrm{ns}} \\
{\left[\mathrm{km} \mathrm{s}^{-2}\right]}\end{array}$ & $\alpha_{\text {gas }}$ & $d_{\text {shock }}$ \\
\hline B12-m1 & 29.7 & 71.5 & 0.11 & 60.9 & 47.4 & 0.36 & 0.226 & 1.384 & -56.8 & -1.7 & -208.2 & 0.06 & 0.48 \\
\hline B12-m2 & 29.7 & 71.5 & 0.11 & 60.9 & 47.7 & 0.31 & 0.222 & 1.385 & -100.0 & 19.1 & -63.5 & 0.10 & 0.72 \\
\hline B12-m3 & 29.7 & 71.5 & 0.11 & 61.2 & 47.8 & 0.38 & 0.210 & 1.388 & 272.6 & -16.5 & 91.9 & 0.23 & 0.35 \\
\hline B12-m4 & 29.7 & 71.5 & 0.11 & 60.9 & 47.0 & 0.35 & 0.209 & 1.378 & -104.3 & -7.4 & -197.2 & 0.09 & 0.43 \\
\hline B12-m5 & 29.7 & 71.5 & 0.11 & 60.8 & 47.9 & 0.35 & 0.219 & 1.389 & 365.6 & -10.1 & 219.1 & 0.32 & 0.47 \\
\hline B12-m6 & 29.7 & 71.5 & 0.11 & 60.7 & 48.4 & 0.36 & 0.229 & 1.395 & -334.1 & 42.4 & -462.9 & 0.30 & 0.26 \\
\hline B18-m1 & 44.5 & 107.3 & 0.16 & 68.9 & 54.9 & 1.12 & 0.136 & 1.274 & 43.3 & -4.8 & -108.8 & 0.02 & 0.12 \\
\hline B18-m2 & 44.5 & 107.3 & 0.16 & 68.9 & 54.8 & 1.14 & 0.139 & 1.273 & -86.8 & -1.1 & -31.1 & 0.03 & 0.20 \\
\hline B18-m3 & 44.5 & 107.3 & 0.16 & 68.8 & 55.3 & 1.12 & 0.131 & 1.281 & 76.4 & -8.8 & -11.4 & 0.03 & 0.39 \\
\hline B18-m4 & 44.5 & 107.3 & 0.16 & 68.5 & 54.9 & 1.14 & 0.150 & 1.274 & -118.7 & 14.5 & -156.4 & 0.05 & 0.13 \\
\hline B18-m5 & 44.5 & 107.3 & 0.16 & 68.3 & 54.7 & 1.12 & 0.166 & 1.273 & -339.7 & -4.5 & -152.4 & 0.13 & -0.06 \\
\hline B18-m6 & 44.5 & 107.3 & 0.16 & 68.6 & 55.4 & 1.12 & 0.166 & 1.283 & -439.3 & 14.0 & -194.5 & 0.17 & 0.04 \\
\hline B18-m7 & 44.5 & 107.3 & 0.16 & 68.8 & 54.7 & 1.12 & 0.138 & 1.272 & 109.2 & 8.6 & 2.1 & 0.04 & 0.38 \\
\hline B18-m8 & 44.5 & 107.3 & 0.16 & 69.3 & 54.5 & 1.13 & 0.134 & 1.269 & 455.0 & -4.1 & 187.4 & 0.17 & 0.05 \\
\hline
\end{tabular}

which has the dimension of a momentum. Then we can write the anisotropy parameter as

$\alpha_{\text {gas }}:=\left|\boldsymbol{P}_{\text {gas }}\right| / P_{\text {ej }}$,

and the absolute value of the neutron star velocity as

$\left|\boldsymbol{v}_{\mathrm{ns}}\right|=\alpha_{\mathrm{gas}} P_{\mathrm{ej}} / M_{\mathrm{ns}}$.

The neutron star acceleration corresponding to the velocity change at a given time is calculated by finite differences:

$\boldsymbol{a}_{\mathrm{ns}}^{(n)}=\frac{\boldsymbol{v}_{\mathrm{ns}}^{(n+1)}-\boldsymbol{v}_{\mathrm{ns}}^{(n-1)}}{t^{(n+1)}-t^{(n-1)}}$.

In computing the recoil velocity according to Eqs. (A.10) and (A.14), we have so far neglected the fact that the neutron star may also be accelerated by anisotropic neutrino emission. While our core luminosities at the inner grid boundary are assumed to be isotropic at all times and no neutron star acceleration can result from these, direction-dependent variations of the thermodynamic variables in layers close to the neutron star surface develop during the simulations and ultimately lead to anisotropies of the neutrinospheric emission of neutrinos. In particular, density inhomogeneities and local hot-spots (in temperature) occur as a consequence of narrow accretion flows that transport gas from the postshock layers to the neutron star, where they are decelerated in shocks and radiate away energy in neutrinos. The anisotropy of this neutrino emission can give rise to a "neutrino rocket effect", whose magnitude can be estimated by considering the integrated momentum of the escaping neutrinos.
For a transport scheme along radial rays like ours, the neutrino momentum density has only a radial component and can thus be written as (see also Appendix D)

$p_{v} \boldsymbol{e}_{\mathrm{r}}=\frac{n_{v} \epsilon_{v}}{c} \boldsymbol{e}_{\mathrm{r}}=\frac{F_{v}}{c^{2}} \boldsymbol{e}_{\mathrm{r}}$,

where $F_{v}$ is the local neutrino energy flux and $\boldsymbol{e}_{\mathrm{r}}$ the unit vector in the radial direction. The integrated neutrino momentum at time $t$ is then given by

$$
\begin{aligned}
\boldsymbol{P}_{v}(t) & =\int_{R_{\mathrm{ib}}<r<\infty} p_{v} \boldsymbol{e}_{\mathrm{r}} \mathrm{d} V \\
& =\int_{R_{\mathrm{ib}}<r<R_{\mathrm{ob}}} p_{\nu} \boldsymbol{e}_{\mathrm{r}} \mathrm{d} V+\int_{0}^{t} \mathrm{~d} t \oint_{r=R_{\mathrm{ob}}} p_{v} c \boldsymbol{e}_{\mathrm{r}} \mathrm{d} S,
\end{aligned}
$$

with the surface element $\mathrm{d} S=r^{2} \sin \theta \mathrm{d} \theta \mathrm{d} \phi$. Here the surface integral accounts for the fact that a significant amount of neutrino momentum may have left our grid through the outer boundary by the time $t$. The momentum of the neutron star, including now also the effect of anisotropic neutrino emission, is

$\boldsymbol{P}_{\mathrm{ns}}=-\left(\boldsymbol{P}_{\mathrm{gas}}+\boldsymbol{P}_{v}\right)$,

so that the neutron star velocity, corrected for the recoil by anisotropic neutrino emission, can be written as

$\boldsymbol{v}_{\mathrm{ns}, \text { corr }}=\boldsymbol{v}_{\mathrm{ns}}+\boldsymbol{v}_{\mathrm{ns}, v}=-\boldsymbol{P}_{\mathrm{gas}} / M_{\mathrm{ns}}-\boldsymbol{P}_{v} / M_{\mathrm{ns}}$.

We finally note that for symmetry reasons $\boldsymbol{P}_{\text {gas }}$ and $\boldsymbol{P}_{v}$, and thus also $\boldsymbol{P}_{\mathrm{ns}}$ and $\boldsymbol{v}_{\mathrm{ns}}$, can have only a component parallel to the 
symmetry axis, i.e. along the $z$-axis, in 2D axisymmetric calculations. Equation (A.11), for instance, therefore reduces to

$$
\begin{aligned}
P_{z, \text { gas }} & =2 \pi \int_{R_{\mathrm{ns}}}^{\infty} \mathrm{d} r \int_{0}^{\pi} \mathrm{d} \theta r^{2} \sin \theta p_{z}(r, \theta) \\
& =2 \pi \int_{R_{\mathrm{ns}}}^{\infty} \mathrm{d} r \int_{0}^{\pi / 2} \mathrm{~d} \theta r^{2} \sin \theta\left[p_{z}(r, \theta)+p_{z}(r, \pi-\theta)\right] \\
& =P_{z, \text { gas }}^{\mathrm{N}}+P_{z, \text { gas }}^{\mathrm{S}} .
\end{aligned}
$$

Here $p_{z}(r, \theta)=\rho\left(v_{r} \cos \theta-v_{\theta} \sin \theta\right)$ is the $z$-component of the momentum density of the gas, and $P_{z \text {,gas }}^{\mathrm{N}}$ and $P_{z \text {,gas }}^{\mathrm{S}}$ are introduced as the $z$-momenta of the gas in the northern and southern hemispheres, respectively.

\section{Appendix B: Hydrodynamics in an accelerated frame of reference}

In an inertial frame of reference the hydrodynamic equations are given by

$$
\begin{aligned}
& \frac{\partial \rho}{\partial t}+\nabla \cdot(\rho \boldsymbol{v})=0, \\
& \rho\left(\frac{\partial \boldsymbol{v}}{\partial t}+(\boldsymbol{v} \cdot \nabla) \boldsymbol{v}\right)+\nabla \mathcal{P}=\rho \boldsymbol{g}, \\
& \frac{\partial \rho E}{\partial t}+\nabla \cdot((\rho E+\mathcal{P}) \boldsymbol{v})=\boldsymbol{v} \cdot \rho \boldsymbol{g},
\end{aligned}
$$

where $\rho$ is the density, $\boldsymbol{v}$ is the velocity, $\mathcal{P}$ is the pressure, $\boldsymbol{g}$ is the gravitational acceleration and $E=\epsilon+v^{2} / 2$ is the sum of internal energy, $\epsilon$, and kinetic energy, $\epsilon_{\text {kin }}$, per unit mass.

Let $\mathrm{AF}$ be a frame of reference that coincides with an inertial frame IF at time $t=0$ and accelerates with a constant rate $a$ in $z$-direction, $\boldsymbol{a}=\boldsymbol{a} \boldsymbol{e}_{z}$. The Cartesian coordinates of both frames are then related by

$\left(x^{\prime}, y^{\prime}, z^{\prime}, t^{\prime}\right)=\left(x, y, z-a t^{2} / 2, t\right)$

(primed quantities are used for the accelerated frame), which implies that

$\partial z^{\prime}(x, y, z, t) / \partial t=-a t \quad$ and $\quad \partial z\left(x^{\prime}, y^{\prime}, z^{\prime}, t^{\prime}\right) / \partial t^{\prime}=a t$.

For density, pressure, velocity, kinetic energy and gravitational acceleration the following relations hold:

$\rho^{\prime}\left(x^{\prime}, y^{\prime}, z^{\prime}, t\right)=\rho(x, y, z, t)$,

$\mathcal{P}^{\prime}\left(x^{\prime}, y^{\prime}, z^{\prime}, t\right)=\mathcal{P}(x, y, z, t)$,

$\boldsymbol{v}^{\prime}\left(x^{\prime}, y^{\prime}, z^{\prime}, t\right)=\boldsymbol{v}(x, y, z, t)-$ ate $_{z}$

$\epsilon_{\mathrm{kin}}^{\prime}\left(x^{\prime}, y^{\prime}, z^{\prime}, t\right)=\epsilon_{\mathrm{kin}}(x, y, z, t)-v_{z}^{2} / 2+\left(v_{z}-a t\right)^{2} / 2$,

$\boldsymbol{g}^{\prime}\left(x^{\prime}, y^{\prime}, z^{\prime}, t\right)=\boldsymbol{g}(x, y, z, t)-a \boldsymbol{e}_{z}$.

From relations (B.4)-(B.6), it is easy to see that the equation of mass conservation (B.1) does not change in the accelerated frame. The momentum equation in this frame is

$\rho^{\prime}\left(\frac{\partial \boldsymbol{v}^{\prime}}{\partial t}+\left(\boldsymbol{v}^{\prime} \cdot \boldsymbol{\nabla}^{\prime}\right) \boldsymbol{v}^{\prime}\right)+\boldsymbol{\nabla}^{\prime} \mathcal{P}^{\prime}-\rho \boldsymbol{g}^{\prime}=-a t \rho\left(\frac{\partial v_{x}}{\partial z} \boldsymbol{e}_{x}+\frac{\partial v_{y}}{\partial z} \boldsymbol{e}_{y}\right)$.

Note that in contrast to Eq. (B.2) there is now an additional term on the right hand side, which affects the momentum components perpendicular to the direction of acceleration. Thus for instance the $x$-component of the time derivative of the velocity is

$$
\frac{\partial v_{x}^{\prime}}{\partial t}=-\left(v_{x}^{\prime} \frac{\partial v_{x}^{\prime}}{\partial x^{\prime}}+v_{y}^{\prime} \frac{\partial v_{x}^{\prime}}{\partial y^{\prime}}+v_{z}^{\prime} \frac{\partial v_{x}^{\prime}}{\partial z^{\prime}}\right)-\frac{1}{\rho^{\prime}} \frac{\partial \mathcal{P}^{\prime}}{\partial x^{\prime}}+g_{x}^{\prime}-a t \frac{\partial v_{x}}{\partial z},
$$

where the additional (rightmost) term is negligible compared to $v_{z}^{\prime}\left(\partial v_{x}^{\prime} / \partial z^{\prime}\right)$, as long as $|a t| \ll\left|v_{z}\right|$.

Similarly, it can be shown that the additional terms arising in the energy Eq. (B.3) for an accelerated frame of reference are of order $t^{2}$ and can also be neglected, as long as $|a t| \ll\left|v_{z}\right|$ holds.

Within a typical time step $\Delta t$ of a supernova simulation (of order $10^{-6} \mathrm{~s}$ ) the condition $|a \Delta t| \ll\left|v_{z}\right|$ is satisfied, because the maximum neutron star accelerations are of $O\left(10^{8} \mathrm{~cm} / \mathrm{s}^{2}\right)$, and hence $|a \Delta t|=O(100 \mathrm{~cm} / \mathrm{s})$, which is much smaller than the relevant velocities in the simulations, which are of $O\left(10^{6} \mathrm{~cm} / \mathrm{s}\right)$. Thus a solution of the inertial frame hydrodynamics equations with the simple replacement $\boldsymbol{g} \rightarrow \boldsymbol{g}^{\prime}=\boldsymbol{g}-\boldsymbol{a}$ should yield an excellent approximation to the solution of the hydrodynamic equations in the accelerated frame.

Unfortunately, in the present problem the neutron star acceleration, and hence the instantaneous acceleration of the frame, $\boldsymbol{a}(t)$, is not known a priori, because it is coupled to the solution of the hydrodynamic problem during a considered time step. Therefore we need to make use of an operator-splitting approach, in which we first ignore the acceleration of the frame of reference and simply solve the inertial frame hydrodynamics equations (just using the gravitational acceleration $\boldsymbol{g}$ ). We can then compute the current value of $\boldsymbol{a}(t)$, which is assumed to be constant over the time step, using momentum conservation: The sum of the momenta of the neutron star core, $\boldsymbol{P}_{\text {core }}$, and the matter on the numerical grid, $\boldsymbol{P}_{\text {grid }}$, is conserved and initially zero, so that $\Delta \boldsymbol{P}_{\text {core }}=-\Delta \boldsymbol{P}_{\text {grid }}$. We can then use the relation

$\boldsymbol{a}\left(t^{n}\right)=-\frac{\boldsymbol{P}_{\text {grid }}\left(t^{n}\right)-\boldsymbol{P}_{\text {grid }}\left(t^{n-1}\right)}{M_{\text {core }} \Delta t}$

to determine the acceleration in this time step. Finally we take the effects of the global acceleration of our frame into account in a second step, by adding

$-\boldsymbol{a}\left(t^{n}\right) \Delta t \equiv-\Delta \boldsymbol{v}_{\mathrm{core}}^{n}$

to the hydrodynamic velocity in each zone of the grid, in essence performing a Galilei transformation to an instantaneous inertial frame in which the neutron star is again at rest.

\section{Appendix C: Explosion energy}

The explosion energy of neutrino-driven supernovae consists of two major contributions. The first is the recombination energy of the matter in the gain layer at the onset of the explosion. This matter consists of free nucleons and alpha particles at the time the explosion starts. Almost all of this mass (except for some fraction in the downflows, which is accreted onto the neutron star) ends up in a dense shell behind the expanding shock. As the shock propagates outward, the temperature in this expanding shell decreases and the matter recombines to $\alpha$-particles and later to nuclei.

Figure C.1 displays the available recombination energy of the matter in the gain layer at the time of the explosion,

$E_{\text {rec }}^{\text {gain }}\left(t_{\text {exp }}\right)=\int_{\text {gain layer }} \epsilon_{\text {rec }}\left(r, t_{\text {exp }}\right) \mathrm{d} V$.

Here $\epsilon_{\mathrm{rec}}(r, t)$ denotes the density of recombination energy available when matter consists of nucleons, $\alpha$-particles and some mass fraction of heavy nuclei,

$\epsilon_{\mathrm{rec}}(r, t)=B_{\mathrm{h}} n_{\mathrm{h}}^{\max }(r, t)-\left(B_{\alpha} n_{\alpha}(r, t)+B_{\mathrm{h}} n_{\mathrm{h}}(r, t)\right)$, 


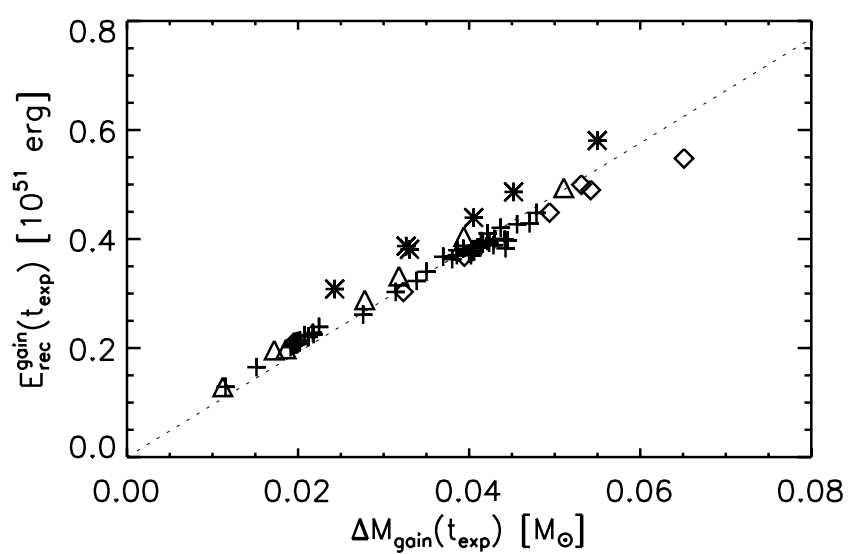

Fig. C.1. Available recombination energy, $E_{\mathrm{rec}}^{\text {gain }}$, as a function of the mass in the gain layer, $\Delta M_{\text {gain }}$, at the time of explosion for the models of Tables A.1-A.5. The slope of this approximately linear relation corresponds to about $5 \mathrm{MeV}$ per baryon (dotted line).

with $B_{\mathrm{h}}$ and $B_{\alpha}$ being the binding energies of a representative heavy nucleus $\left(Z_{\mathrm{h}}, A_{\mathrm{h}}=N_{\mathrm{h}}+Z_{\mathrm{h}}\right)$ from the iron group (as assumed in our equation of state) and of $\alpha$-particles, respectively, and $n_{\mathrm{h}}^{\max }=\min \left(n_{\mathrm{p}}^{\text {tot }} / Z_{\mathrm{h}}, n_{\mathrm{n}}^{\text {tot }} / N_{\mathrm{h}}\right)$ and $n_{\mathrm{h}}$ are the maximum and current number densities, respectively, of this heavy nucleus when $n_{\mathrm{p}}^{\text {tot }}$ and $n_{\mathrm{n}}^{\text {tot }}$ are the total (bound+free) number densities of protons and neutrons.

Figure C.1 shows that for all models of Tables A.1-A.5 $E_{\text {rec }}^{\text {gain }}\left(t_{\text {exp }}\right) \approx N_{\mathrm{b}}^{\text {gain }}\left(t_{\text {exp }}\right) \times 5 \mathrm{MeV}$, when $N_{\mathrm{b}}^{\text {gain }}$ is the total number of baryons in the gain layer. This means that due to the partial assembly of free $\mathrm{n}$ and $\mathrm{p}$ in $\alpha$-particles at the time of explosion, about $5 \mathrm{MeV}$ (instead of $>8 \mathrm{MeV}$ ) remain available for being released by recombination during the subsequent expansion and cooling.

This recombination is essentially complete when the shock has reached a radius of $3000 \mathrm{~km}$ (recombination to $\alpha$-particles happens even much earlier). We denote this time by $t_{\text {rec }}$. Figure C.2 demonstrates that the explosion energy at time $t_{\mathrm{rec}}$, $E_{\text {exp }}\left(t_{\text {rec }}\right)$, roughly equals the available recombination energy, $E_{\mathrm{rec}}^{\mathrm{gain}}\left(t_{\mathrm{exp}}\right)$, at the onset of the explosion. This means that neutrino heating essentially has the effect of lifting the total energy of mass elements in the gain layer close to zero (i.e., $\epsilon_{\mathrm{tot}}=\epsilon_{\mathrm{kin}}+\epsilon_{\mathrm{int}}+\epsilon_{\mathrm{grav}} \approx 0$ ) and thus makes this matter unbound and enables its expansion in the gravitational potential of the forming neutron star. The excess energy of this matter at time $t_{\mathrm{rec}}$, i.e. $E_{\mathrm{exp}}\left(t_{\mathrm{rec}}\right)$, is provided by the recombination of nucleons to $\alpha$-particles and finally to iron-group nuclei. Only in case of higher explosion energies, $E_{\exp }\left(t_{\text {rec }}\right)$ is clearly larger than $E_{\text {rec }}^{\text {gain }}\left(t_{\text {exp }}\right)$ (Fig. C.2). In this case neutrino heating in the gain layer is stronger and the heating time scale of the matter there shorter than the expansion time scale when the shock begins to accelerate outwards. Therefore neutrinos are able to deposit "excess energy" in the ejecta before this matter has moved out of the region of strong heating.

The second contribution to the explosion energy comes from the neutrino-driven baryonic wind which sets in after the surroundings of the nascent neutron star have been cleaned from the initially heated gas. Indeed this wind is an important energy source at "late" times. To demonstrate this, we compare the time derivative of the explosion energy, $\mathrm{d} E_{\exp } / \mathrm{d} t$, with the wind power, $L_{\text {wind }}$, and the net energy loss/gain rate $L_{\text {shock }}$ at the

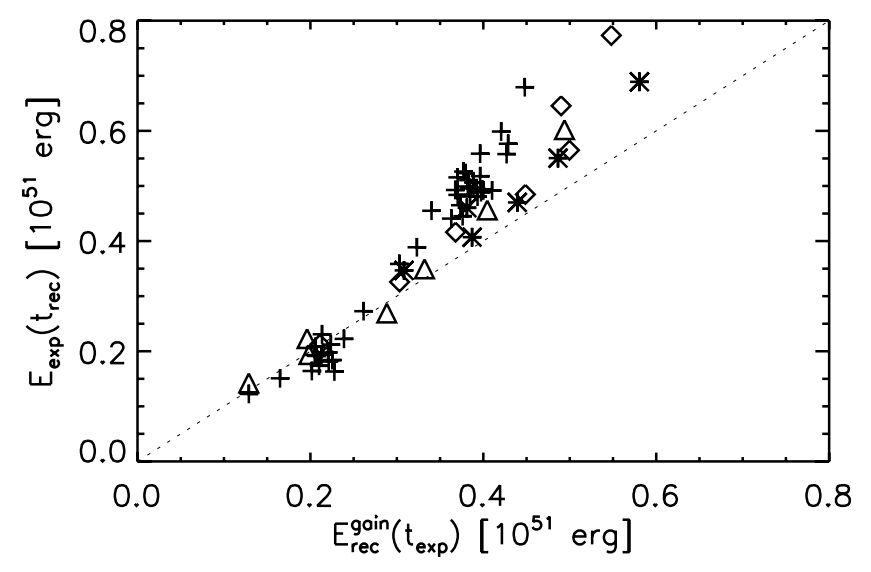

Fig. C.2. Explosion energy after the recombination of the ejecta, $E_{\text {exp }}\left(t_{\text {rec }}\right)$, as a function of the available recombination energy in the gain layer at the onset of the explosion, $E_{\mathrm{rec}}^{\text {gain }}\left(t_{\mathrm{exp}}\right)$, for the models of Tables A.1-A.5. For low explosion energies the two quantities agree well.

shock (Fig. C.3). The curve for $\mathrm{d} E_{\text {exp }} / \mathrm{d} t$ in Fig. C.3 is calculated as the numerical derivative of the energy integral

$E_{\exp }(t)=\int_{V^{+}} \epsilon_{\mathrm{tot}}(r, t) \mathrm{d} V$

where the integration is performed over the volume $V^{+}$, in which the total energy $\epsilon_{\mathrm{tot}}(r, t)$ is positive (see also Eq. (A.5)). For $t>$ $t_{\mathrm{rec}}$ this volume fills the region between an inner boundary at $r \approx 200 \mathrm{~km}$ and the shock (except for some parts of the accretion downflows, where $\epsilon_{\text {tot }}$ may still be negative).

The explosion energy is subject to changes by $\mathcal{P} \mathrm{d} V$-work performed at, and by energy fluxes through the boundaries of $V^{+}$, in particular by the wind, whose power is given by the surface integral

$L_{\mathrm{wind}}=\oint_{r=200 \mathrm{~km}}\left(\epsilon_{\mathrm{tot}}+\epsilon_{\mathrm{rec}}+\mathcal{P}\right) \max \left(v_{r}, 0\right) \mathrm{dS}$.

This expression takes into account the total energy $\left(\epsilon_{\mathrm{tot}}=\rho e_{\mathrm{tot}}\right.$ with $e_{\text {tot }}$ defined by Eq. (A.6)) of the wind material streaming through the inner boundary radius into $V^{+}$, the energy that will be set free at larger radii by recombination (Eq. (C.1)), as well as the work performed by pressure forces. Here we have neglected effects due to downflows by omitting contributions to the surface integral from zones with negative radial velocity.

The change of the explosion energy due to energy flow through the outer boundary of $V^{+}$(i.e., the shock), is given by the net energy loss/gain rate

$L_{\mathrm{shock}}=\oint_{r=R_{\mathrm{s}}(\theta)^{+}}\left[\left(\epsilon_{\mathrm{tot}}+\epsilon_{\mathrm{rec}}+\mathcal{P}\right) v_{r}+\left(\epsilon_{\mathrm{tot}}+\epsilon_{\mathrm{rec}}\right) \dot{R}_{\mathrm{s}}\right] \mathrm{dS}$.

The integration has to be performed over a surface located slightly upstream of the shock. Compared to Eq. (C.4) an additional term arises here from the motion of the shock, which propagates with a local velocity $\dot{R}_{\mathrm{s}}(\theta)$.

Figure C. 3 shows that these two terms explain the evolution of $\mathrm{d} E_{\exp } / \mathrm{d} t$ for $t>t_{\mathrm{rec}}$, i.e.

$\mathrm{d} E_{\text {exp }} / \mathrm{d} t \approx L_{\text {wind }}+L_{\text {shock }}$

holds at late times, and the thin and thick solid lines in Fig. C.3 almost coincide. Note also that $L_{\text {wind }} \gg\left|L_{\text {shock }}\right|$. This is true for all our models, and therefore the increase of the explosion energy 


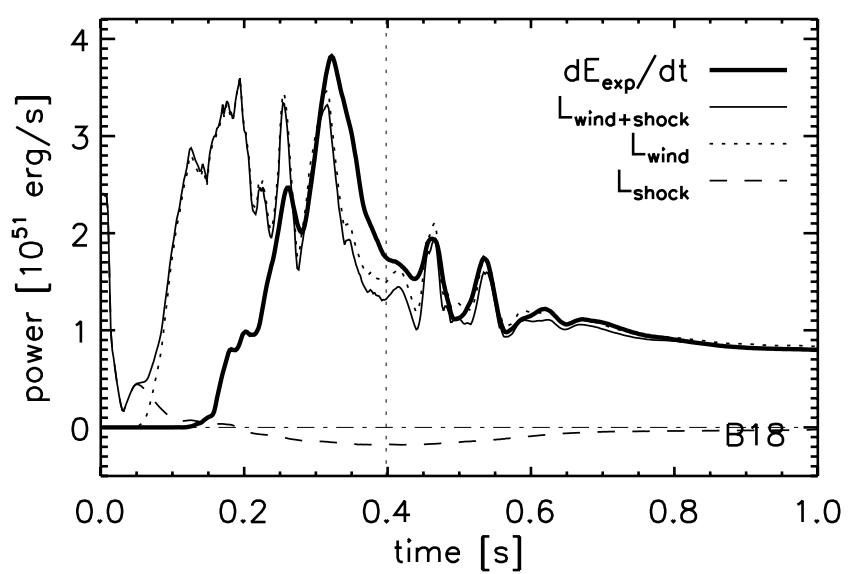

Fig. C.3. Evolution of the time derivative of the explosion energy $\left(\mathrm{d} E_{\exp } / \mathrm{d} t\right.$, thick solid) for model B18. Also shown are the wind power at a radius of $200 \mathrm{~km}$ ( $L_{\text {wind }}$, dotted), the energy loss/gain rate at the shock by $\mathcal{P} \mathrm{d} V$ work and swept-up matter ( $L_{\text {shock}}$, dashed), and the sum of the latter two quantities ( $L_{\text {wind+shock}}$, thin solid). $L_{\text {wind+shock }}$ agrees well with $\mathrm{d} E_{\exp } / \mathrm{d} t$ for $t>t_{\text {rec }}$ (right of the vertical line).

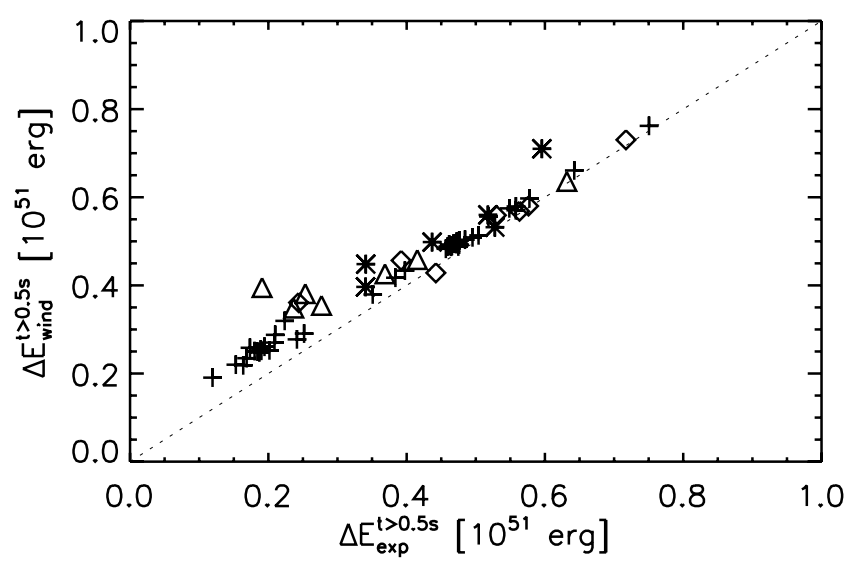

Fig. C.4. Relation between the increase of the explosion energy between $t=0.5 \mathrm{~s}$ and $t=1 \mathrm{~s}, \Delta E_{\exp }^{t>0.5 \mathrm{~s}}$, and the integrated wind power during this time interval, $\Delta E_{\text {wind }}^{t>0.5 \mathrm{~s}}$, for the models of Tables A.1-A.5.

after about $0.5 \mathrm{~s}$ post bounce is (almost exclusively) associated with the time-integrated wind power (see Fig. C.4).

The relative importance of the two major constituents of the explosion energy that we have discussed here, i.e., the nuclear recombination energy of the matter in the gain layer and the integrated power of the neutrino-driven wind, varies with the explosion energy. In our "standard boundary contraction" models the fraction of the explosion energy provided by recombination drops from about $70 \%$ for the low-energy models to about $30 \%$ for the model with $E_{\exp }(1 \mathrm{~s}) \approx 1.5 \times 10^{51} \mathrm{erg}$ (Fig. C.5). This fraction declines because the wind power is proportional to a higher power of the luminosity ( $L_{\text {wind }} \propto L_{v}^{\alpha}$ with $\alpha \approx 3$; Thompson et al. 2001) and although the mass in the gain layer at the onset of the explosion scales linearly with the boundary luminosity (Fig. 10).

For the "rapid boundary contraction" cases the wind contribution is even more important, e.g. for model W12F-c $E_{\mathrm{rec}}^{\text {gain }}\left(t_{\exp }\right) / E_{\text {exp }}(1 \mathrm{~s}) \approx 0.2$, i.e. about $80 \%$ of the explosion energy are generated by the neutrino-driven wind. For a fixed boundary luminosity the wind power is higher in this case than for the "standard boundary contraction", because $L_{\text {wind }}$ increases with decreasing neutron star radius (see e.g. Thompson et al. 2001). However, $\Delta M_{\text {gain }}\left(t_{\exp }\right)$, and thus also $E_{\text {rec }}^{\text {gain }}\left(t_{\exp }\right)$, are

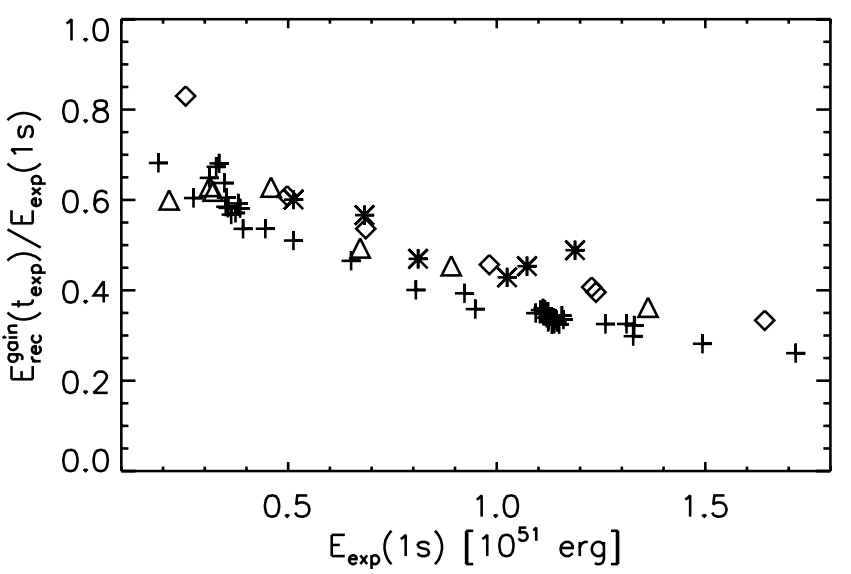

Fig. C.5. Ratio of the recombination contribution to the total explosion energy $1 \mathrm{~s}$ after core bounce, $E_{\mathrm{rec}}^{\mathrm{gain}}\left(t_{\exp }\right) / E_{\exp }(1 \mathrm{~s})$, as a function of $E_{\text {exp }}(1 \mathrm{~s})$ for the models of Tables A.1-A.5. For low-energy models the recombination contribution dominates, whereas for higher explosion energies the wind contribution becomes more important.

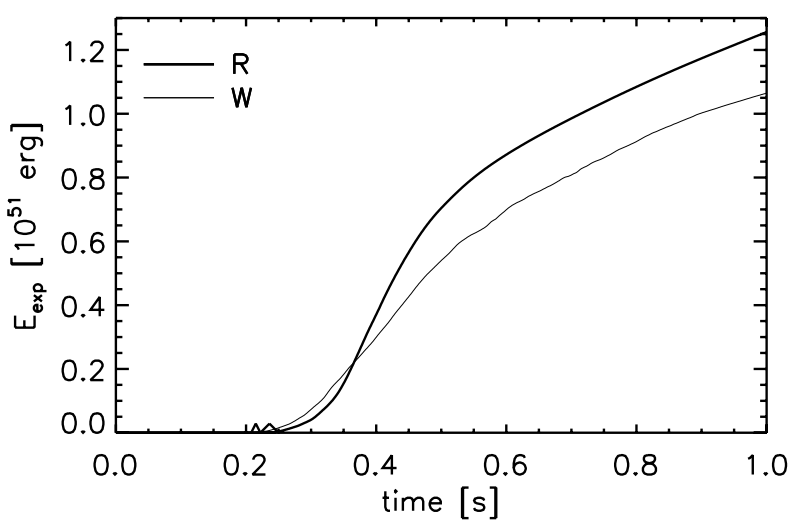

Fig. C.6. Evolution of the explosion energy for models R18-c and W18-c which are listed in Table 2. The rotating model R18-c attains an explosion energy which is higher than in the non-rotating case W18-c due to a larger gain layer mass at the time of explosion. Note also that the explosion energy in both cases is still increasing at $t=1 \mathrm{~s}$.

similar for models with "standard" and "rapid" boundary contraction and the same $L_{\mathrm{ib}}$. This is so because two effects compensate each other roughly: On the one hand the density at a given radius $r$ in the gain layer is lower for a faster contraction $\left(\rho^{\mathrm{r}}\left(r, t_{\text {exp }}^{\mathrm{r}}\right)<\rho^{\mathrm{s}}\left(r, t_{\text {exp }}^{\mathrm{s}}\right)\right.$, where $\mathrm{r}$ and s denote the rapid and standard contraction cases, respectively), but on the other hand also the gain radius is smaller and thus located in a region of higher density, $\rho^{\mathrm{r}}\left(R_{\mathrm{g}}^{\mathrm{r}}, t_{\mathrm{exp}}^{\mathrm{r}}\right)>\rho^{\mathrm{s}}\left(R_{\mathrm{g}}^{\mathrm{s}}, t_{\mathrm{exp}}^{\mathrm{s}}\right)$.

Figure C.6 indicates that the explosion energy is still increasing at $t=1 \mathrm{~s}$ when we stopped most of our simulations. Yet, with the subsequent drop of the core luminosity (we assume a $t^{-3 / 2}$ behaviour at $t>t_{L}$, see Eq. (D.11)) also the wind power, which is proportional to $L_{v}^{\alpha}$ (see above), must decline strongly. Therefore the explosion energy will grow only moderately. In case of the long-time simulation B18-lt it rose from $1.14 \times 10^{51} \mathrm{erg}$ at $t=1 \mathrm{~s}$ to $1.43 \times 10^{51} \mathrm{erg}$ at $t=2 \mathrm{~s}$, and reached $1.46 \times 10^{51} \mathrm{erg}$ by the end of the simulation at $t=3.6 \mathrm{~s}$. 


\section{Appendix D: Neutrino transport}

\section{D.1. Transport equation}

We start from the equation of radiation transport in spherical symmetry

$$
\frac{1}{c} \frac{\partial}{\partial t} I+\mu \frac{\partial}{\partial r} I+\frac{1-\mu^{2}}{r} \frac{\partial}{\partial \mu} I=S,
$$

where $I=I(t, r, \epsilon, \mu)$ is the specific intensity, $S=S(t, r, \epsilon, \mu)$ is the source function, $\epsilon$ is the neutrino energy, $\mu=\cos \theta$ and $\theta$ is the angle between radiation propagation and radial direction. Solid angle integration yields the zeroth angular moment equation,

$$
\frac{1}{c} \frac{\partial}{\partial t} J+\frac{1}{r^{2}} \frac{\partial}{\partial r}\left(r^{2} H\right)=S^{(0)} \equiv \frac{1}{2} \int_{-1}^{+1} \mathrm{~d} \mu S
$$

with $\{J, H\}(t, r, \epsilon):=\frac{1}{2} \int_{-1}^{+1} \mathrm{~d} \mu \mu^{\{0,1\}} I(t, r, \epsilon, \mu)$. Integration over energy leads to

$$
\frac{\partial}{\partial t} E+\frac{1}{r^{2}} \frac{\partial}{\partial r}\left(r^{2} F\right)=Q^{+}-Q^{-}
$$

with $\{E, F\}(t, r):=4 \pi \int_{0}^{\infty} \mathrm{d} \epsilon\{J / c, H\}(t, r, \epsilon)$ being energy density and energy flux, respectively. The source term has been split in an emission rate $Q^{+}$and an absorption rate $Q^{-}=\kappa_{\mathrm{a}} c E$, which is proportional to the energy density. The flux factor is defined as the ratio of flux to energy density,

$f(r, t):=F(r, t) / c E(r, t)$.

In neutrino transport simulations solving the full Boltzmann equation (see e.g. Buras et al. 2003, 2006a,b) this quantity shows only little short-time variability during most phases of the supernova evolution. Therefore $\partial f / \partial t=0$ is an acceptably good approximation. With $L:=4 \pi r^{2} F=4 \pi r^{2} f c E$ one can now rewrite Eq. (D.3) as

$$
\frac{\partial}{\partial t} L+c_{\mathrm{eff}} \frac{\partial}{\partial r} L=4 \pi r^{2} c_{\mathrm{eff}}\left\{Q^{+}-Q^{-}\right\}
$$

where an effective speed of neutrino propagation has been introduced as $c_{\text {eff }}:=c f$. Provided $c_{\text {eff }}$ were known, the solution of Eq. (D.5) requires considerably less effort than the numerical integration of Eq. (D.1). For vanishing source terms $Q^{+}$and $Q^{-}$the neutrino energy or number density is just advected along characteristics $r(t)=r_{0}+c_{\text {eff }} t$. Although $c_{\text {eff }}$ depends through $f(r, t)$ on the solution of the transport problem (Eq. (D.4)), neutrino transport calculations in the neutrino-decoupling layer of forming neutron stars reveal that it can be well fitted by a $r$-dependent function which depends on the steepness of the density profile (see Janka 1990, 1991b). Assuming further that the (mediumdependent) coefficients $Q^{+}$and $\tilde{\kappa} \equiv \kappa_{\mathrm{a}} / f=4 \pi r^{2} Q^{-} / L$ are constant between two points $(r, t)$ and $\left(r^{\star}, t^{\star}\right)$, which are connected by a characteristic line, i.e.,

$r^{\star}=r-c_{\mathrm{eff}}\left(t-t^{\star}\right)$,

Eq. (D.5) can be integrated analytically to yield

$$
\begin{aligned}
L(r, t)= & L\left(r^{\star}, t^{\star}\right) \mathrm{e}^{-\tilde{\kappa} c_{\text {eff }}\left(t-t^{\star}\right)} \\
& +\frac{4 \pi Q^{+}}{\tilde{\kappa}^{3}}\left\{\left[1-\mathrm{e}^{-\tilde{\kappa} c_{\text {eff }}\left(t-t^{\star}\right)}\right]\left[1+\left(\tilde{\kappa} r^{\star}-1\right)^{2}\right]\right. \\
& \left.+\tilde{\kappa} c_{\text {eff }}\left(t-t^{\star}\right)\left[2 \tilde{\kappa} r^{\star}+\tilde{\kappa} c_{\text {eff }}\left(t-t^{\star}\right)-2\right]\right\},
\end{aligned}
$$

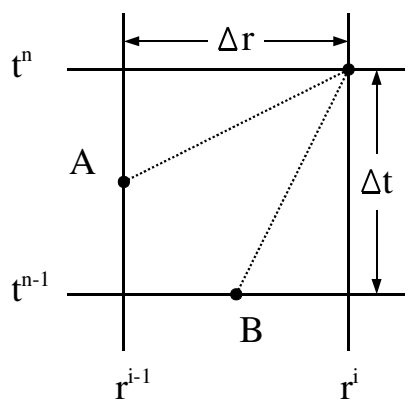

Fig. D.1. The solution at $\left(r^{i}, t^{n}\right)$ is computed from the data at a point $\left(r^{\star}, t^{\star}\right)$ located on the same characteristic line. Depending on the grid spacing, $\Delta r$, the time step, $\Delta t$, and the effective speed of neutrino propagation, $c_{\text {eff }}$, either point $\mathrm{A}$ or point $\mathrm{B}$ must be used. The solution there can be obtained by interpolation in time or space, respectively.

where $L(r, t)$ and $L\left(r^{\star}, t^{\star}\right)$ are the luminosity values at both ends of the characteristic line.

We use Eq.(D.7) to construct a numerical scheme to solve Eq. (D.5) in the general case: We assume that the luminosity is known at the cell interfaces of a one-dimensional radial grid for a time $t^{n-1}$, and that the cell-averaged values of the quantities needed to compute the emission rate $Q^{+}$and absorption coefficient $\tilde{\kappa}$ are also known for that time. As a further simplification we do not allow neutrinos to propagate in negative radial direction (actually this is granted by defining a non-negative function for the flux factor, see Sect. D.3). Then the luminosities at $t^{n}=t^{n-1}+\Delta t$ for each zone interface (starting at the innermost zone) can be computed using Eq. (D.7). In doing so we have to distinguish between two cases (see Fig. D.1): If $c_{\text {eff }} \Delta t>\Delta r$, we can use point $\mathrm{A}$ as the starting point of the integration, $\left(r^{\star}, t^{\star}\right)=\left(r_{i-1}, t_{A}\right)$. The luminosity at this point is derived from a linear interpolation between $L\left(r_{i-1}, t^{n-1}\right)$ and $L\left(r_{i-1}, t^{n}\right)$ (which is already known, as we are integrating outwards). If $c_{\text {eff }} \Delta t \leq \Delta r$, we use point $\mathrm{B}$, the luminosity at this point being given by a linear interpolation between $L\left(r_{i-1}, t^{n-1}\right)$ and $L\left(r_{i}, t^{n-1}\right)$.

For time integration we use a predictor-corrector method: The transport routine is called two times. In the first (predictor) step the luminosities, emission rates and absorption coefficients of the last time step $\left[L^{n-1}, Q^{n-1}, \kappa^{n-1}\right]$ are used to compute preliminary values $\left(\tilde{Q}^{n}, \tilde{\kappa}^{n}\right)$ for the neutrino-medium coupling at the next time level. In the second (corrector) step the final values $\left[L^{n}, Q^{n}, \kappa^{n}\right]$ are calculated using $\left[L^{n-1}, \frac{1}{2}\left(Q^{n-1}+\tilde{Q}^{n}\right), \frac{1}{2}\left(\kappa^{n-1}+\right.\right.$ $\left.\left.\tilde{\kappa}^{n}\right)\right]$ as input.

Equation (D.5) is solved not only for the energy luminosity $L=L_{e}$, but also for the number luminosity $L_{n}=4 \pi r^{2} F_{n}=$ $4 \pi r^{2} f c n$ ( $n$ is the particle density and $f$ is assumed to be the same flux factor as for the energy transport). Furthermore the equation has to be integrated for three neutrino types, $v_{\mathrm{e}}, \bar{v}_{\mathrm{e}}$, and $v_{x}$ (the latter denoting $v_{\mu}, \bar{v}_{\mu}, v_{\tau}$, and $\bar{v}_{\tau}$, which are treated identically). In the following we will use indices $v \in\left\{v_{\mathrm{e}}, \bar{v}_{\mathrm{e}}, v_{x}\right\}$ and $\alpha \in\{e, n\}$ to distinguish between these different cases.

In the $2 \mathrm{D}$ case the neutrino transport is assumed to proceed only radially, i.e. lateral components of the neutrino flux are ignored and Eq. (D.5) is integrated independently on different radial "rays", i.e. in radial direction for every lateral zone of the polar coordinate grid. Total luminosities of the star are obtained by summing up the flux densities $L / 4 \pi r^{2}$ for all angular cells (at a given radius $r$ ), appropriately weighting them with the corresponding surface elements. 


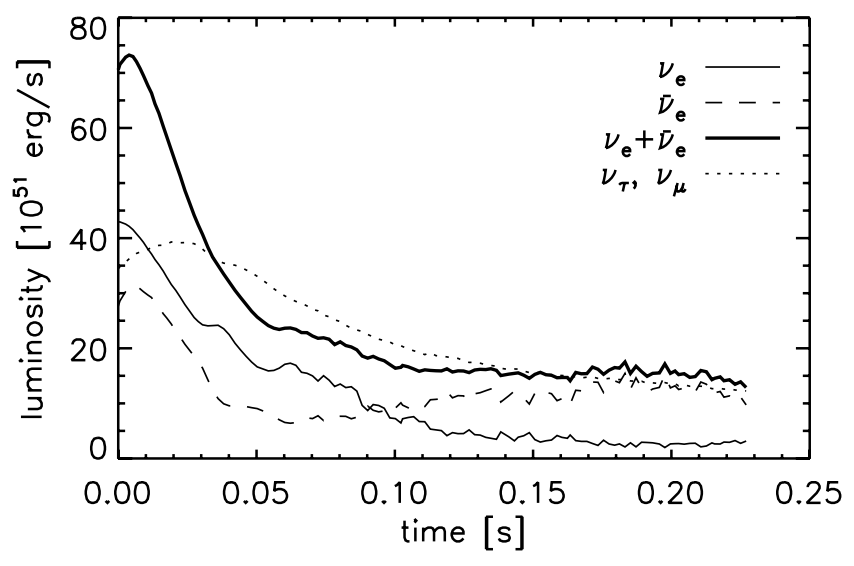

Fig. D.2. Evolution after core bounce of the neutrino luminosities at a Lagrangian mass shell of $1.1 M_{\odot}$ from a supernova simulation with Boltzmann neutrino transport (Buras et al. 2003). After an initial phase of $50 \mathrm{~ms}$ duration, the sum of the $v_{\mathrm{e}}$ and $\bar{v}_{\mathrm{e}}$ luminosities, as well as the $v_{\mu} / v_{\tau}$ luminosities vary only slowly.

\section{D.2. Boundary conditions}

To integrate Eq. (D.7) outwards, time-dependent boundary conditions are required for the luminosities $L_{\mathrm{e}, v_{i}}$ and $L_{\mathrm{n}, v_{i}}$, where $v_{i}=v_{\mathrm{e}}, \bar{v}_{\mathrm{e}}, v_{x}$. We assume $L_{\mathrm{e}, v_{i}}$ to be constant for a time interval $t_{L}$ (typically $1 \mathrm{~s}$ ), and to decay subsequently with a power-law dependence in time:

$L_{\mathrm{e}, v_{\mathrm{e}}}\left(R_{\mathrm{ib}}, t\right)=L_{v}^{\mathrm{tot}, 0} K_{v_{\mathrm{e}}} h(t)$,

$L_{\mathrm{e}, \bar{\nu}_{\mathrm{e}}}\left(R_{\mathrm{ib}}, t\right)=L_{v}^{\mathrm{tot}, 0} K_{\bar{v}_{\mathrm{e}}} h(t)$,

$L_{\mathrm{e}, v_{x}}\left(R_{\mathrm{ib}}, t\right)=L_{v}^{\mathrm{tot}, 0} K_{v_{x}} h(t)$,

where

$h(t)=\left\{\begin{array}{lll}1.0 & \text { if } & t \leq t_{L}, \\ \left(t_{L} / t\right)^{3 / 2} & \text { if } & t>t_{L} .\end{array}\right.$

The constants $K_{v_{i}}$ denote the fractional contributions of the individual luminosities to the total neutrino luminosity. They fulfill the requirement

$K_{v_{\mathrm{e}}}+K_{\bar{v}_{\mathrm{e}}}+4 K_{v_{x}}=1$.

The functional form used in Eq. (D.11) can be motivated by the Boltzmann transport calculations of Buras et al. (2003). These show that after a transient phase of $\sim 50 \mathrm{~ms}$, which is short compared to the explosion time scales of our simulations, the sum of all luminosities is almost constant or varies only very weakly, at least over the next $\sim 250 \mathrm{~ms}$, for which data from the Boltzmann transport simulations are available (see Fig. D.2).

According to Eqs. ((D.8)-(D.11)) we need to prescribe the time scale $t_{L}$ and the total initial luminosity $L_{v}^{\text {tot, } 0}$. However, instead of choosing these two quantities as basic parameters of our models, we prefer to prescribe $t_{L}$ and the gravitational binding energy $\Delta E_{v, \text { core }}^{\infty}$ that is released by the neutron star core asymptotically (i.e. for $t \rightarrow \infty$ ) via neutrino emission. Introducing the energy that the core looses up to time $t$

$\Delta E_{v, \mathrm{core}}^{\mathrm{tot}}(t)=\int_{0}^{t} L_{v}^{\mathrm{tot}, 0} h\left(t^{\prime}\right) \mathrm{d} t^{\prime}$

the following relations hold for the asymptotic energy loss

$\Delta E_{v, \text { core }}^{\infty}=\int_{0}^{\infty} L_{v}^{\mathrm{tot}, 0} h(t) \mathrm{d} t=3 \Delta E_{v, \text { core }}^{\mathrm{tot}}\left(t_{L}\right)=3 L_{v}^{\mathrm{tot}, 0} t_{L}$, i.e. our ansatz of Eq. (D.11) implies that $1 / 3$ of $\Delta E_{v, \text { core }}^{\infty}$ is radiated away within the chosen time interval $t_{L}$ in neutrinos and antineutrinos of all flavours.

We also prescribe the mean energies of neutrinos entering the computational grid at the inner boundary. The corresponding values are chosen to be $\left\langle\epsilon_{v_{\mathrm{e}}}\right\rangle^{\mathrm{ib}}=12 \mathrm{MeV},\left\langle\epsilon_{\overline{\mathrm{v}}_{\mathrm{e}}}\right\rangle^{\mathrm{ib}}=16 \mathrm{MeV}$, and $\left\langle\epsilon_{v_{x}}\right\rangle^{\mathrm{ib}}=20 \mathrm{MeV}$, and kept constant during our simulations. Thereby also the number fluxes $L_{n, v_{i}}=L_{e, v_{i}} /\left\langle\epsilon_{v_{i}}\right\rangle$ at $r=R_{\mathrm{ib}}$ are defined.

The total lepton number lost by the neutron star core until time $t$, normalised to the total baryon number $N_{\mathrm{b} \text {, core }}$ of the core, is given by

$\Delta Y_{\mathrm{e}, \text { core }}(t)=N_{\mathrm{b}, \text { core }}^{-1} \int_{0}^{t}\left(L_{n, v_{\mathrm{e}}}\left(R_{\mathrm{ib}}, t^{\prime}\right)-L_{n, \bar{v}_{\mathrm{e}}}\left(R_{\mathrm{ib}}, t^{\prime}\right)\right) \mathrm{d} t^{\prime}$.

For $t=t_{\mathrm{L}}$ this yields

$\Delta Y_{\mathrm{e}, \text { core }}\left(t_{L}\right)=\frac{L_{v}^{\mathrm{tot}, 0} t_{\mathrm{L}}}{N_{\mathrm{b}, \text { core }}}\left(\frac{K_{v_{\mathrm{e}}}}{\left\langle\epsilon_{\overline{\mathrm{e}}_{\mathrm{e}}}\right\rangle^{\mathrm{ib}}}-\frac{K_{\bar{\nu}_{\mathrm{e}}}}{\left\langle\epsilon_{\overline{\mathrm{v}}_{\mathrm{e}}}\right\rangle^{\mathrm{ib}}}\right)$.

We assume that the lepton number loss during time interval $t_{\mathrm{L}}$ is proportional to the energy loss during this time. Therefore we choose $K_{v_{i}}=$ const, because $\left\langle\epsilon_{v_{i}}\right\rangle^{\text {ib }}=$ const, and set $K_{v_{\mathrm{e}}}=0.2$, $K_{\bar{v}_{\mathrm{e}}}=0.215$ for the calculations in this paper. $K_{v_{x}}$ follows from Eq. (D.12).

\section{D.3. Neutrino distribution function}

To calculate the source terms in Eqs. (D.5) or (D.7) we have to make an assumption about the neutrino energy spectrum, i.e. about the energy dependency of the specific intensity, which for particle energy and particle number is linked with the particle distribution function $f_{\mathrm{D}, v}$ in the following way:

$I_{v,\{\mathrm{n}, \mathrm{e}\}}(t, r, \epsilon, \mu)=\left(\frac{\epsilon^{\{2,3\}}}{(h c)^{3}}\right) c f_{\mathrm{D}, v}(t, r, \epsilon, \mu)$,

where the exponent of 2 applies for number transport and the exponent of 3 for energy transport, corresponding to the indices $n$ and e, respectively, of $I_{v}$. We assume that $f_{\mathrm{D}, v}$ can be written as product of a Fermi-Dirac distribution function,

$f_{\mathrm{FD}}(x, \eta)=\frac{1}{1+\exp (x-\eta)}$,

and an angle-dependent function $g_{v}$,

$f_{\mathrm{D}, v}(r, t, \epsilon, \mu)=g_{v}(r, t, \mu) f_{\mathrm{FD}}\left(\frac{\epsilon}{k_{\mathrm{B}} T_{v}(r, t)}, \eta_{v}(r, t)\right)$,

where in general the spectral temperature and degeneracy parameter, $T_{v}$ and $\eta_{v}$, are different from the matter temperature and equilibrium degeneracy parameter.

Furthermore we assume that $\eta_{v}$ is just a function of the optical depth $\tau_{v}$ :

$\eta_{v}\left(\tau_{v}\right)=\eta_{\mathrm{eq}, v}\left(1-\mathrm{e}^{-\tau_{v}}\right)+\eta_{0, v} \mathrm{e}^{-\tau_{v}}$,

where $\eta_{\mathrm{eq}, v}$ is the equilibrium degeneracy parameter and $\eta_{0, v}$ is a chosen value as typically found in detailed transport calculations for $\tau_{v} \rightarrow 0$. The values for the different neutrino types are (cf. Janka 1991b; Janka \& Hillebrandt 1989; Myra \& Burrows 1990; Keil et al. 2003):

$\eta_{\mathrm{eq}, v_{\mathrm{e}}}=\left(\mu_{\mathrm{e}}+\mu_{\mathrm{p}}-\mu_{\mathrm{n}}\right) / k_{\mathrm{B}} T$,

$\eta_{0, v_{\mathrm{e}}} \equiv 3$

$\eta_{\mathrm{eq}, \bar{v}_{\mathrm{e}}}=-\eta_{\mathrm{eq}, v_{\mathrm{e}}}, \quad \eta_{0, \bar{v}_{\mathrm{e}}} \equiv 2$,

$\eta_{\mathrm{eq}, v_{x}}=0$, 
With $\eta_{v}$ defined, $T_{v}$ can now be calculated from the local average neutrino energy, which is computed from $L_{\mathrm{e}, v}$ and $L_{\mathrm{n}, v}$ as

$$
\begin{aligned}
\left\langle\epsilon_{v}\right\rangle & =L_{\mathrm{e}, v} / L_{\mathrm{n}, v}=E_{v}(r, t) / n_{v}(r, t) \\
& =\frac{\int_{0}^{\infty} \mathrm{d} \epsilon \int_{-1}^{+1} \mathrm{~d} \mu I_{\mathrm{e}, v}(r, t, \epsilon, \mu)}{\int_{0}^{\infty} \mathrm{d} \epsilon \int_{-1}^{+1} \mathrm{~d} \mu I_{\mathrm{n}, v}(r, t, \epsilon, \mu)} \\
& =k_{\mathrm{B}} T_{v} \mathcal{F}_{3}\left(\eta_{v}\right) / \mathcal{F}_{2}\left(\eta_{v}\right)
\end{aligned}
$$

with the Fermi integrals defined by

$\mathcal{F}_{n}(\eta) \equiv \int_{0}^{\infty} \mathrm{d} x x^{n} f_{\mathrm{FD}}(x, \eta)$

Thus the energy-dependent part of $f_{\mathrm{D}, v}$ is fully defined. The angle-dependent part is related to the flux factor by

$f_{v}=F_{v} / c E_{v}=\frac{\int_{-1}^{+1} \mathrm{~d} \mu \mu I_{v}}{\int_{-1}^{+1} \mathrm{~d} \mu I_{v}}=\frac{\int_{-1}^{+1} \mathrm{~d} \mu \mu g_{v}(\mu)}{\int_{-1}^{+1} \mathrm{~d} \mu g_{v}(\mu)}=\left\langle\mu_{v}\right\rangle$.

To solve Eq. (D.5) only $f_{v}$ is needed, not the angle-dependent function $g_{v}(\mu)$. Far outside of the neutrinosphere, $f_{v}$ is approaching the vacuum solution. The latter can be derived under the assumption that neutrinos are emitted isotropically from the sharp surface of a sphere with radius $R_{v}$, which is located at a distance $r$ from the observer. In this case the flux factor is

$f_{v, \mathrm{vac}}=\frac{1}{2}\left[1+\sqrt{1-\left(R_{v} / r\right)^{2}}\right]$.

$f_{v \text {,vac }}$ approaches 1 for $r \rightarrow \infty$ (free streaming limit) and $f_{v \text {,vac }}=$ $1 / 2$ at the neutrinosphere. In a more realistic situation the neutrinosphere is not a sharp surface but a layer with finite thickness in which neutrinos gradually decouple from the stellar medium. In detailed transport calculations $f_{v}\left(R_{v}\right)$ is therefore found to be about 1/4. (see e.g. Janka \& Hillebrandt 1989; Janka 1990). How fast $f_{v}$ approaches $f_{v \text {,vac }}$ (with declining optical depth) depends on the steepness of the density gradient at the neutrinosphere (Janka 1990). Inside the neutrinosphere detailed transport calculations show that the flux factor behaves roughly like $f_{v} \propto \tau_{v}^{m}$ with $m<0$.

Taking all this into account, the following function constitutes a good approximation for the flux factors from detailed transport calculations (Janka 1991b, 1990):

$f_{v}\left(\tau_{v}\right)=\left\{\begin{array}{lll}\frac{\frac{1}{2}[1+D]}{1+(1+D)\left(1-D^{2}\right)^{(n-1) / 2},}, & \text { if } & \tau_{v}<\tau_{v, 1}, \\ 1 / 4\left(\tau_{v} / \tau_{v, 1}\right)^{m}, & \text { if } & \tau_{v}>\tau_{v, 1} .\end{array}\right.$

Here $D=\sqrt{1-\left(R_{v} / r\right)^{2}}$, the neutrinosphere radius is defined by $\tau_{v}\left(R_{v}\right)=\tau_{v, 1}$ and we adopt $\tau_{v, 1}=1$.1. The power-law index $m$ is chosen such that $f_{v}(10)=1 / 25$, and $n$ is defined by a local power-law fit of the density profile around the neutrinosphere, $\rho(r) \propto r^{-n}$. A higher value of $n$ therefore means a steeper density gradient.

\section{D.4. Neutrino reactions}

For calculating the neutrino-matter interaction rates the following reactions are taken into account: charged-current processes with neutrons (n) and protons (p),

$v_{\mathrm{e}}+\mathrm{n} \rightleftharpoons \mathrm{p}+\mathrm{e}^{-}$,

$\bar{v}_{\mathrm{e}}+\mathrm{p} \rightleftharpoons \mathrm{n}+\mathrm{e}^{+}$, thermal electron-positron $\left(\mathrm{e}^{ \pm}\right)$pair creation and annihilation,

$\mathrm{e}^{+}+\mathrm{e}^{-} \rightleftharpoons v_{i}+\bar{v}_{i} \quad(i=\mathrm{e}, \mu, \tau)$

and neutrino scattering off nuclei (A), nucleons, and electrons and positrons,

$$
\begin{gathered}
v_{i}+\mathrm{A} \rightleftharpoons v_{i}+\mathrm{A}, \\
v_{i}+\left\{\begin{array}{l}
\mathrm{n} \\
\mathrm{p}
\end{array}\right\} \rightleftharpoons v_{i}+\left\{\begin{array}{l}
\mathrm{n} \\
\mathrm{p}
\end{array}\right\}, \\
v_{i}+\mathrm{e}^{ \pm} \rightleftharpoons v_{i}+\mathrm{e}^{ \pm} .
\end{gathered}
$$

\section{D.5. Optical depth}

Knowledge of the optical depth is necessary to evaluate Eqs. (D.20) and (D.26). For this purpose it is sufficient to compute $\tau_{v}$ approximately by considering only the most relevant neutrino processes and assuming that the neutrino spectrum is given by the spectrum for local thermodynamic equilibrium. This means that instead of Eq. (D.19) we use

$f_{\mathrm{D}, v}^{\mathrm{eq}}\left(\epsilon_{v}, r\right)=f_{\mathrm{FD}}\left(\frac{\epsilon_{v}}{k_{\mathrm{B}} T(r)}, \eta_{\mathrm{eq}, v}(r)\right)$

with $\eta_{\mathrm{eq}, v}$ and $T$ instead of $\eta_{v}$ and $T_{v}$.

The "transport optical depth" is defined as the integral

$\tau_{\mathrm{t}, v}(r)=\int_{r}^{\infty} \mathrm{d} r^{\prime}\left\langle\kappa_{\mathrm{t}, v}\right\rangle\left(r^{\prime}\right)$

of the energy-averaged "transport opacity" (i.e. the opacity which is relevant for momentum transfer), $\left\langle\kappa_{\mathrm{t}, v}\right\rangle(r)$ (see, e.g., Straumann 1989; Burrows \& Thompson 2004). In the following, all neutrino interactions included in evaluating the opacity are calculated without final-state lepton blocking, unless otherwise stated.

The most important opacity-producing reactions are scattering off nucleons $(\mathrm{n}, \mathrm{p})$ and nuclei $\left(Z_{j}, A_{j}\right)$, where $j=1,2, \ldots$ denotes the considered nuclear species, and absorption by neutrons and protons in case of $v_{\mathrm{e}}$ and $\bar{v}_{\mathrm{e}}$, respectively. Thus one can write

$\left\langle\kappa_{\mathrm{t}, v}\right\rangle=\left\langle\kappa_{v}^{\mathrm{a}}\right\rangle+\sum_{i \in\left\{\mathrm{n}, \mathrm{p}, A_{j}\right\}}\left\langle\kappa_{\mathrm{t}, v}^{\mathrm{s}, i}\right\rangle$.

Here the (neutrino-flavour independent) scattering opacities are to lowest order in neutrino energy over nucleon rest mass (i.e., without effects of nucleon recoil, thermal motions, and weakmagnetism corrections):

$$
\begin{aligned}
\left\langle\kappa_{\mathrm{t}, v}^{\mathrm{s}, \mathrm{p}}\right\rangle= & \frac{1}{6}\left[\frac{5}{4} \alpha^{2}+\left(C_{V}-1\right)^{2}\right] \frac{\sigma_{0}}{\left(m_{\mathrm{e}} c^{2}\right)^{2}}\left\langle\epsilon_{v}^{2}\right\rangle n_{\mathrm{p}}, \\
\left\langle\kappa_{\mathrm{t}, v}^{\mathrm{s}, \mathrm{n}}\right\rangle= & \frac{5 \alpha^{2}+1}{24} \frac{\sigma_{0}}{\left(m_{\mathrm{e}} c^{2}\right)^{2}}\left\langle\epsilon_{v}^{2}\right\rangle n_{\mathrm{n}}, \\
\left\langle\kappa_{\mathrm{t}, v}^{\mathrm{s}, A_{j}}\right\rangle= & \frac{1}{6} A_{j}^{2}\left[C_{A}-1+\frac{Z_{j}}{A_{j}}\left(2-C_{A}-C_{V}\right)\right]^{2} \\
& \times \frac{\sigma_{0}}{\left(m_{\mathrm{e}} c^{2}\right)^{2}}\left\langle\epsilon_{v}^{2}\right\rangle n_{A_{j}},
\end{aligned}
$$

for scattering off protons, neutrons, and nuclei with number densities $n_{\mathrm{p}}, n_{\mathrm{n}}$, and $n_{A_{j}}$, respectively (see, e.g., Freedman et al. 
1977; Straumann 1989; Burrows \& Thompson 2004). The absorption opacities for $v_{\mathrm{e}}$ and $\bar{v}_{\mathrm{e}}$ are

$$
\begin{aligned}
\left\langle\kappa_{v_{\mathrm{e}}}^{\mathrm{a}}\right\rangle= & \frac{1}{4}\left(3 \alpha^{2}+1\right) \frac{\sigma_{0}}{\left(m_{\mathrm{e}} c^{2}\right)^{2}} n_{\mathrm{n}} \\
& \times\left(\left\langle\epsilon_{v_{\mathrm{e}}}^{2}\right\rangle+2 \Delta\left\langle\epsilon_{v_{\mathrm{e}}}\right\rangle+\Delta^{2}\right) \Theta\left(\left\langle\epsilon_{v_{\mathrm{e}}}\right\rangle\right), \\
\left\langle\kappa_{\bar{v}_{\mathrm{e}}}^{\mathrm{a}}\right\rangle= & \frac{1}{4}\left(3 \alpha^{2}+1\right) \frac{\sigma_{0}}{\left(m_{\mathrm{e}} c^{2}\right)^{2}} n_{\mathrm{p}} \\
& \times\left(\left\langle\epsilon_{\bar{\nu}_{\mathrm{e}}}^{2}\right\rangle^{\star}+2 \Delta\left\langle\epsilon_{\bar{\nu}_{\mathrm{e}}}\right\rangle^{\star}+\Delta^{2}\left\langle\epsilon_{\bar{\nu}_{\mathrm{e}}}^{0}\right\rangle^{\star}\right) .
\end{aligned}
$$

(see Tubbs \& Schramm 1975; Bruenn 1985). Here $\sigma_{0}=$ $4 G_{\mathrm{F}}^{2} m_{\mathrm{e}}^{2} \hbar^{2} / \pi c^{2}=1.76 \times 10^{-44} \mathrm{~cm}^{2}$ (with the Fermi coupling constant $\left.G_{\mathrm{F}}\right), \Delta=1.2935 \mathrm{MeV}$ is the rest mass difference of neutrons and protons, $\alpha=1.254, C_{A}=\frac{1}{2}, C_{V}=\frac{1}{2}+2 \sin ^{2} \theta_{\mathrm{W}}$, and $\sin ^{2} \theta_{\mathrm{W}}=0.23$

In deriving Eqs. (D.36)-(D.40) (as well as for all rates and source terms given below) the electron and positron rest masses are ignored $\left(m_{\mathrm{e}} c^{2} \ll \epsilon_{v}\right)$ and nucleons and nuclei are assumed to have infinite rest masses $\left(m_{\mathrm{n}, \mathrm{p}, A_{j}} c^{2} \gg \epsilon_{v}\right)$ and to be nondegenerate. For electrons, phase space blocking is included in Eq. (D.40) by the factor

$\Theta\left(\left\langle\epsilon_{v}\right\rangle\right)=1-f_{\mathrm{FD}}\left(\frac{\left\langle\epsilon_{v}\right\rangle+\Delta}{k_{\mathrm{B}} T}, \eta_{\mathrm{e}^{-}}\right)$,

which accounts for the fact that a significant fraction of the possible final electron states may be occupied. Phase space blocking can be neglected in $\kappa_{\bar{v}_{\mathrm{e}}}^{\mathrm{a}}$ (Eq. (D.40)), because the positrons are non-degenerate.

The neutrino energy moments are (generalising Eq. (D.22)) given by

$\left\langle\epsilon_{v}^{n}\right\rangle=\left(k_{\mathrm{B}} T_{v}\right)^{n} \frac{\mathcal{F}_{2+n}\left(\eta_{v}\right)}{\mathcal{F}_{2}\left(\eta_{v}\right)}$,

$\left\langle\epsilon_{v}^{n}\right\rangle^{\star}=\left(k_{\mathrm{B}} T_{v}\right)^{n} \frac{\mathcal{F}_{2+n}\left(\eta_{v}-\Delta / k_{\mathrm{B}} T_{v}\right)}{\mathcal{F}_{2}\left(\eta_{v}\right)}$,

and for evaluating Eqs. (D.36)-(D.40) to compute $\tau_{\mathrm{t}, v}$ (Eq. (D.34)) for use in Eq. (D.26), we take $\eta_{v}=\eta_{\mathrm{eq}, v}$ and $T_{v}=T$.

In contrast, Eq. (D.20) is evaluated with the "effective optical depth for equilibration",

$\tau_{v}(r)=\int_{r}^{\infty} \mathrm{d} r^{\prime}\left\langle\kappa_{\mathrm{eff}, \nu}\right\rangle\left(r^{\prime}\right)$

where the effective opacity is defined as

$\left\langle\kappa_{\mathrm{eff}, \nu}\right\rangle=\sqrt{\left\langle\kappa_{v}^{\mathrm{a}}\right\rangle \times\left\langle\kappa_{v}^{\mathrm{a}}+\kappa_{\mathrm{t}, \nu}^{\mathrm{s}}\right\rangle}$.

Here the spectrally averaged absorption opacity, $\left\langle\kappa_{\gamma}^{\mathrm{a}}\right\rangle$, is taken to include neutrino-pair annihilation to $\mathrm{e}^{ \pm}$-pairs (Eq. (D.29)), which is assumed to be the most important reaction for producing $v_{x} \bar{v}_{x}$ pairs. Both $\left\langle\kappa_{v}^{\mathrm{a}}\right\rangle$ and $\left\langle\kappa_{v}^{\mathrm{a}}+\kappa_{\mathrm{t}, v}^{\mathrm{s}}\right\rangle$ are evaluated for the "true" (not the local equilibrium) neutrino spectrum (i.e. for the spectral temperature $T_{v}$ and the spectral degeneracy $\eta_{v}$ instead of $T$ and $\eta_{\mathrm{eq}, v}$ ) by employing the source terms from the neutrino transport solution of the last time step.

\section{D.6. Source terms}

Solving Eq. (D.7) requires the knowledge of the emission rates, $Q_{v_{i}}^{+}$, and absorption coefficients, $\tilde{\kappa}_{v}=\kappa_{v}^{\mathrm{a}} / f_{v}$, which appear in this equation. Since Eq. (D.7) is used to determine the number fluxes, $L_{n, v}$, and luminosities, $L_{\mathrm{e}, v}$, of all neutrinos and antineutrinos $v \in\left\{v_{\mathrm{e}}, \bar{v}_{\mathrm{e}}, v_{x}\right\}$, the source terms need to be calculated for the neutrino number, as well as energy. In the following, all these neutrino source terms are derived without taking into account final-state lepton blocking, unless otherwise stated. As in Eq. (D.45), $\kappa_{v}^{\mathrm{a}}$ is defined to include the contributions from the $\beta$-processes, Eqs. (D.27) and (D.28) for $\nu_{\mathrm{e}}$ and $\bar{v}_{\mathrm{e}}$, as well as those of $\mathrm{e}^{+} \mathrm{e}^{-}$pair annihilation (Eq. (D.29)). The absorption coefficient $\kappa_{v}^{\mathrm{a}}$ can be computed from the corresponding neutrino absorption rate by

$\kappa_{v}^{\mathrm{a}}=Q_{v}^{-} 4 \pi r^{2} f_{v} / L_{v}=\left(Q_{v}^{\mathrm{a}}+Q_{v \bar{v}}^{\mathrm{ann}}\right) 4 \pi r^{2} f_{v} / L_{v}$.

For the number transport the neutrino absorption and emission rates (in units of number per $\mathrm{cm}^{3}$ per second) by charged-current $\beta$-reactions between leptons and nucleons can be written with our approximations for the neutrino distribution function and the appropriate statistical weights for the leptons as follows:

$\mathcal{R}_{v_{\mathrm{e}}}^{\mathrm{a}}=\sigma c \frac{L_{\mathrm{e}, v_{\mathrm{e}}} n_{\mathrm{n}}}{4 \pi r^{2} c f_{v_{\mathrm{e}}}} \frac{\left\langle\epsilon_{v_{\mathrm{e}}}^{2}\right\rangle+2 \Delta\left\langle\epsilon_{v_{\mathrm{e}}}\right\rangle+\Delta^{2}}{\left\langle\epsilon_{v_{\mathrm{e}}}\right\rangle} \Theta\left(\left\langle\epsilon_{v_{\mathrm{e}}}\right\rangle\right)$,

$\mathcal{R}_{\bar{v}_{\mathrm{e}}}^{\mathrm{a}}=\sigma c \frac{L_{\mathrm{e}, \overline{\mathrm{v}}_{\mathrm{e}}} n_{\mathrm{p}}}{4 \pi r^{2} c f_{\overline{\mathrm{v}}_{\mathrm{e}}}} \frac{\left\langle\epsilon_{\bar{v}_{\mathrm{e}}}^{2}\right\rangle^{\star}+2 \Delta\left\langle\epsilon_{\overline{\bar{v}}_{\mathrm{e}}}\right\rangle^{\star}+\Delta^{2}\left\langle\epsilon_{\bar{v}_{\mathrm{e}}}^{0}\right\rangle^{\star}}{\left\langle\epsilon_{\bar{v}_{\mathrm{e}}}\right\rangle}$,

$\mathcal{R}_{v_{\mathrm{e}}}^{\mathrm{e}}=\frac{1}{2} \sigma c n_{\mathrm{p}} n_{\mathrm{e}^{-}}\left[\left\langle\epsilon_{\mathrm{e}^{-}}^{2}\right\rangle^{\star}+2 \Delta\left\langle\epsilon_{\mathrm{e}^{-}}\right\rangle^{\star}+\Delta^{2}\left\langle\epsilon_{\mathrm{e}^{-}}^{0}\right\rangle^{\star}\right]$,

$\mathcal{R}_{\overline{\mathrm{v}}_{\mathrm{e}}}^{\mathrm{e}}=\frac{1}{2} \sigma c n_{\mathrm{n}} n_{\mathrm{e}^{+}}\left[\left\langle\epsilon_{\mathrm{e}^{+}}^{2}\right\rangle+2 \Delta\left\langle\epsilon_{\mathrm{e}^{+}}\right\rangle+\Delta^{2}\right]$,

where $\sigma=\frac{1}{4}\left(3 \alpha^{2}+1\right) \sigma_{0} /\left(m_{\mathrm{e}} c^{2}\right)^{2}$ and the electron (positron) number density is

$n_{\mathrm{e}^{\mp}}=\frac{8 \pi}{(h c)^{3}}\left(k_{\mathrm{B}}\right)^{3} \mathcal{F}_{2}\left( \pm \eta_{\mathrm{e}^{-}}\right)$.

The electron and positron energy moments are given by

$$
\begin{aligned}
\left\langle\epsilon_{\mathrm{e}}^{n}\right\rangle & =\left(k_{\mathrm{B}} T\right)^{n} \frac{\mathcal{F}_{2+n}\left(\eta_{\mathrm{e}}\right)}{\mathcal{F}_{2}\left(\eta_{\mathrm{e}}\right)}, \\
\left\langle\epsilon_{\mathrm{e}}^{n}\right\rangle^{\star} & =\left(k_{\mathrm{B}} T\right)^{n} \frac{\mathcal{F}_{2+n}\left(\eta_{\mathrm{e}}-\Delta / k_{\mathrm{B}} T\right)}{\mathcal{F}_{2}\left(\eta_{\mathrm{e}}\right)} .
\end{aligned}
$$

The annihilation and production rates of neutrino number in $\mathrm{e}^{+} \mathrm{e}^{-}$pair reactions are given by (adapted from Schinder et al. 1987; see also Janka 1991a and Janka 1991b, and references therein):

$$
\begin{aligned}
\mathcal{R}_{v}^{\mathrm{ann}}= & \frac{\sigma_{0} c}{\left(4 \pi r^{2} c\right)^{2}} \frac{L_{\mathrm{n}, v} L_{\mathrm{n}, \bar{v}}}{\left\langle\epsilon_{v}\right\rangle\left\langle\epsilon_{\bar{v}}\right\rangle}\left\{\frac{2}{9} \frac{\Phi\left(f_{v}, \chi_{v}\right)}{f_{v} f_{\bar{v}}} \frac{C_{\mathrm{A} v}^{2}+C_{\mathrm{V} v}^{2}}{\left(m_{\mathrm{e}} c^{2}\right)^{2}}\left\langle\epsilon_{v}\right\rangle\left\langle\epsilon_{\bar{v}}\right\rangle\right. \\
& \left.+\frac{1}{6} \frac{1-f_{v} f_{\bar{v}}}{f_{v} f_{\bar{v}}}\left(2 C_{\mathrm{V} v}^{2}-C_{\mathrm{A} v}^{2}\right)\right\},
\end{aligned}
$$

$\mathcal{R}_{v}^{\text {prod }}=\frac{1}{18} \frac{\sigma_{0} c}{\left(m_{\mathrm{e}} c^{2}\right)^{2}} n_{\mathrm{e}^{-}} n_{\mathrm{e}^{+}}\left\{\left(C_{\mathrm{A} v}^{2}+C_{\mathrm{V} v}^{2}\right)\left\langle\epsilon_{\mathrm{e}^{-}}\right\rangle\left\langle\epsilon_{\mathrm{e}^{+}}\right\rangle\right.$

$$
\left.+\frac{3}{4}\left(m_{\mathrm{e}} c^{2}\right)^{2}\left(2 C_{\mathrm{V} v}^{2}-C_{\mathrm{A} v}^{2}\right)\right\} \text {. }
$$

These rates hold for neutrinos $v$ or antineutrinos $\bar{v}$ of all flavours. In Eq. (D.46), $\mathcal{R}_{v}^{\mathrm{a}}$ and $\mathcal{R}_{v}^{\mathrm{ann}}$ have to be used instead of $Q_{v}^{\mathrm{a}}$ and $Q_{v}^{\mathrm{ann}}$ for computing the absorption coefficient for the number transport. In Eq. (D.54), $\Phi\left(f_{v}, \chi_{v}\right)$ is a geometrical factor,

$\Phi\left(f_{v}, \chi_{v}\right)=\frac{3}{4}\left[1-2 f_{v} f_{\bar{v}}+\chi_{v} \chi_{\bar{v}}+\frac{1}{2}\left(1-\chi_{v}\right)\left(1-\chi_{\bar{v}}\right)\right]$ 
where we express the variable Eddington factor $\chi_{v}$ in terms of the flux factor $f_{v}$ (Eq. (D.4)) using a statistical form, which was derived by Minerbo (1978) on grounds of maximum entropy considerations (for photons or nondegenerate neutrinos, as assumed here):

$\chi_{v}=\left\langle\mu_{v}^{2}\right\rangle=\frac{1}{3}+\frac{0.01932 f_{v}+0.2694 f_{v}^{2}}{1-0.5953 f_{v}+0.02625 f_{v}^{2}}$.

The weak coupling constants in Eqs. (D.54) and (D.55) are given by

$C_{\mathrm{A} v}=\left\{\begin{array}{l}+\frac{1}{2} \quad \text { for } v \in\left\{v_{\mathrm{e}}, \bar{v}_{\mathrm{e}}\right\}, \\ -\frac{1}{2} \text { for } \quad v \in\left\{v_{\mu}, \bar{v}_{\mu}, v_{\tau}, \bar{v}_{\tau}\right\},\end{array}\right.$

$C_{\mathrm{V} v}=\left\{\begin{array}{l}+\frac{1}{2}+2 \sin ^{2} \theta_{\mathrm{W}} \quad \text { for } \quad v \in\left\{v_{\mathrm{e}}, \bar{v}_{\mathrm{e}}\right\}, \\ -\frac{1}{2}+2 \sin ^{2} \theta_{\mathrm{W}} \text { for } \quad v \in\left\{v_{\mu}, \bar{v}_{\mu}, v_{\tau}, \bar{v}_{\tau}\right\}\end{array}\right.$

The source term which describes the rate of change per unit of volume in the evolution equation of the electron lepton number of the stellar medium is

$Q_{\mathrm{N}}=\dot{Y}_{\mathrm{e}} n_{\mathrm{b}}=\left(\mathcal{R}_{v_{\mathrm{e}}}^{\mathrm{a}}-\mathcal{R}_{v_{\mathrm{e}}}^{\mathrm{e}}\right)-\left(\mathcal{R}_{\bar{v}_{\mathrm{e}}}^{\mathrm{a}}-\mathcal{R}_{\bar{v}_{\mathrm{e}}}^{\mathrm{e}}\right)$.

The source terms which account for the absorption and emission of energy through $v_{\mathrm{e}}$ and $\bar{v}_{\mathrm{e}}$ are computed in analogy to Eqs. (D.47)-(D.50) as

$$
\begin{aligned}
Q_{v_{\mathrm{e}}}^{\mathrm{a}}= & \sigma c \frac{L_{\mathrm{e}, v_{\mathrm{e}}} n_{\mathrm{n}}}{4 \pi r^{2} c f_{v_{\mathrm{e}}}} \frac{\left\langle\epsilon_{v_{\mathrm{e}}}^{3}\right\rangle+2 \Delta\left\langle\epsilon_{v_{\mathrm{e}}}^{2}\right\rangle+\Delta^{2}\left\langle\epsilon_{v_{\mathrm{e}}}\right\rangle}{\left\langle\epsilon_{v_{\mathrm{e}}}\right\rangle} \Theta\left(\left\langle\epsilon_{v_{\mathrm{e}}}\right\rangle\right), \\
Q_{\bar{v}_{\mathrm{e}}}^{\mathrm{a}}= & \sigma c \frac{L_{\mathrm{e}, \bar{v}_{\mathrm{e}}} n_{\mathrm{p}}}{4 \pi r^{2} c f_{\bar{v}_{\mathrm{e}}}} \\
& \times \frac{\left\langle\epsilon_{\overline{\mathrm{v}}_{\mathrm{e}}}^{3}\right\rangle^{\star}+3 \Delta\left\langle\epsilon_{\bar{v}_{\mathrm{e}}}^{2}\right\rangle^{\star}+3 \Delta^{2}\left\langle\epsilon_{\bar{v}_{\mathrm{e}}}\right\rangle^{\star}+\Delta^{3}\left\langle\epsilon_{\bar{\nu}_{\mathrm{e}}}^{0}\right\rangle^{\star}}{\left\langle\epsilon_{\bar{v}_{\mathrm{e}}}\right\rangle}, \\
Q_{v_{\mathrm{e}}}^{\mathrm{e}}= & \frac{\sigma c}{2} n_{\mathrm{p}} n_{\mathrm{e}^{-}}\left[\left\langle\epsilon_{\mathrm{e}^{-}}^{3}\right\rangle^{\star}+2 \Delta\left\langle\epsilon_{\mathrm{e}^{-}}^{2}\right\rangle^{\star}+\Delta^{2}\left\langle\epsilon_{\mathrm{e}^{-}}\right\rangle^{\star}\right], \\
Q_{\bar{v}_{\mathrm{e}}}^{\mathrm{e}}= & \frac{\sigma c}{2} n_{\mathrm{n}} n_{\mathrm{e}^{+}}\left[\left\langle\epsilon_{\mathrm{e}^{+}}^{3}\right\rangle+3 \Delta\left\langle\epsilon_{\mathrm{e}^{+}}^{2}\right\rangle+3 \Delta^{2}\left\langle\epsilon_{\mathrm{e}^{+}}\right\rangle+\Delta^{3}\right] .
\end{aligned}
$$

The annihilation or production of energy in neutrinos $(v)$ by $\mathrm{e}^{+} \mathrm{e}^{-}$pair reactions is given as (Janka 1991a)

$$
\begin{aligned}
Q_{v}^{\mathrm{ann}}= & \frac{\sigma_{0} c}{\left(4 \pi r^{2} c\right)^{2}} \frac{L_{\mathrm{e}, v} L_{\mathrm{e}, \bar{v}}}{\left\langle\epsilon_{v}\right\rangle\left\langle\epsilon_{\bar{v}}\right\rangle}\left\{\frac{2}{9} \frac{\Phi\left(f_{v}, \chi_{v}\right)}{f_{v} f_{\bar{v}}} \frac{C_{\mathrm{A} v}^{2}+C_{\mathrm{V} v}^{2}}{\left(m_{\mathrm{e}} c^{2}\right)^{2}}\left\langle\epsilon_{v}^{2}\right\rangle\left\langle\epsilon_{\bar{v}}\right\rangle\right. \\
& \left.+\frac{1}{6} \frac{1-f_{v} f_{\bar{v}}}{f_{v} f_{\bar{v}}}\left(2 C_{\mathrm{V} v}^{2}-C_{\mathrm{A} v}^{2}\right)\left\langle\epsilon_{v}^{2}\right\rangle\right\}, \\
Q_{v}^{\text {prod }}= & \frac{1}{36} \frac{\sigma_{0} c}{\left(m_{\mathrm{e}} c^{2}\right)^{2}} n_{\mathrm{e}^{-}} n_{\mathrm{e}^{+}} \\
& \times\left\{\left[\left\langle\epsilon_{\mathrm{e}^{-}}^{2}\right\rangle\left\langle\epsilon_{\mathrm{e}^{+}}\right\rangle+\left\langle\epsilon_{\mathrm{e}^{+}}^{2}\right\rangle\left\langle\epsilon_{\mathrm{e}^{-}}\right\rangle\right]\left(C_{\mathrm{A} v}^{2}+C_{\mathrm{V} v}^{2}\right)\right. \\
& \left.+\frac{3}{4}\left(m_{\mathrm{e}} c^{2}\right)^{2}\left[\left\langle\epsilon_{\mathrm{e}^{-}}\right\rangle+\left\langle\epsilon_{\mathrm{e}^{+}}\right\rangle\right]\left(2 C_{\mathrm{V} v}^{2}-C_{\mathrm{A} v}^{2}\right)\right\} .
\end{aligned}
$$

For annihilation of antineutrino $(\bar{v})$ energy, $\left\langle\epsilon_{v}^{2}\right\rangle$ has to be replaced by $\left\langle\epsilon_{\bar{v}}^{2}\right\rangle$ and $\left\langle\epsilon_{v}\right\rangle$ has to be exchanged with $\left\langle\epsilon_{\bar{v}}\right\rangle$ in Eq. (D.65), while the production of $v$ and $\bar{v}$ was assumed to be symmetric and both rates are given by Eq. (D.66).

Also in scattering processes energy can be exchanged between neutrinos and the stellar medium. For scattering off $\mathrm{e}^{-}$
Table D.1. Weak coupling constants for $v$ and $\bar{v}$ scattering off $\mathrm{e}^{+}$or $\mathrm{e}^{-}$ (cf. Eq. (D.67)). $C_{3}^{\mathrm{x}}$ stands for $C_{3}^{x}=\left(C_{\mathrm{A}}-1\right)^{2}-\left(C_{\mathrm{V}}-1\right)^{2}, C_{\mathrm{A}}=\frac{1}{2}$, $C_{\mathrm{V}}=\frac{1}{2}+2 \sin ^{2} \theta_{\mathrm{W}}$, and $v_{x}$ can be $v_{\mu}$ or $v_{\tau}$.

\begin{tabular}{l|ccc}
\hline \hline & $C_{1}$ & $C_{2}$ & $C_{3}$ \\
\hline$v_{\mathrm{e}} \mathrm{e}^{-}$ & $\left(C_{\mathrm{V}}+C_{\mathrm{A}}\right)^{2}$ & $\left(C_{\mathrm{V}}-C_{\mathrm{A}}\right)^{2}$ & $C_{\mathrm{A}}^{2}-C_{\mathrm{V}}^{2}$ \\
$v_{\mathrm{e}} \mathrm{e}^{+}$ & $\left(C_{\mathrm{V}}-C_{\mathrm{A}}\right)^{2}$ & $\left(C_{\mathrm{V}}+C_{\mathrm{A}}\right)^{2}$ & $C_{\mathrm{A}}^{2}-C_{\mathrm{V}}^{2}$ \\
$\bar{v}_{\mathrm{e}} \mathrm{e}^{-}$ & $\left(C_{\mathrm{V}}-C_{\mathrm{A}}\right)^{2}$ & $\left(C_{\mathrm{V}}+C_{\mathrm{A}}\right)^{2}$ & $C_{\mathrm{A}}^{2}-C_{\mathrm{V}}^{2}$ \\
$\bar{v}_{\mathrm{e}} \mathrm{e}^{+}$ & $\left(C_{\mathrm{V}}+C_{\mathrm{A}}\right)^{2}$ & $\left(C_{\mathrm{V}}-C_{\mathrm{A}}\right)^{2}$ & $C_{\mathrm{A}}^{2}-C_{\mathrm{V}}^{2}$ \\
$v_{x} \mathrm{e}^{-}$ & $\left(C_{\mathrm{V}}+C_{\mathrm{A}}-2\right)^{2}$ & $\left(C_{\mathrm{V}}-C_{\mathrm{A}}\right)^{2}$ & $C_{3}^{x}$ \\
$v_{x} \mathrm{e}^{+}$ & $\left(C_{\mathrm{V}}-C_{\mathrm{A}}\right)^{2}$ & $\left(C_{\mathrm{V}}+C_{\mathrm{A}}-2\right)^{2}$ & $C_{3}^{x}$ \\
$\bar{v}_{x} \mathrm{e}^{-}$ & $\left(C_{\mathrm{V}}-C_{\mathrm{A}}\right)^{2}$ & $\left(C_{\mathrm{V}}+C_{\mathrm{A}}-2\right)^{2}$ & $C_{3}^{x}$ \\
$\bar{v}_{x} \mathrm{e}^{+}$ & $\left(C_{\mathrm{V}}+C_{\mathrm{A}}-2\right)^{2}$ & $\left(C_{\mathrm{V}}-C_{\mathrm{A}}\right)^{2}$ & $C_{3}^{x}$ \\
\hline
\end{tabular}

or $\mathrm{e}^{+}$, using the rates of Tubbs \& Schramm (1975), and ignoring electron phase space blocking in the final reaction channels, the following spectrally averaged expression for the energy transfer rate per unit of volume can be derived (see Janka 1991b):

$$
\begin{aligned}
Q_{v \mathrm{e}}= & \frac{1}{12}\left(C_{1}+\frac{1}{6} C_{2}\right) \frac{\sigma_{0} c}{\left(m_{\mathrm{e}} c^{2}\right)^{2}} n_{\mathrm{e}} \frac{L_{e, v}}{4 \pi r^{2} c f_{v}\left\langle\epsilon_{v}\right\rangle} \\
& \left\{\left[\left\langle\epsilon_{v}^{2}\right\rangle\left(\left\langle\epsilon_{\mathrm{e}}\right\rangle+\frac{3}{4} m_{\mathrm{e}} c^{2}\right)-\left\langle\epsilon_{v}\right\rangle\left\langle\epsilon_{\mathrm{e}}^{2}\right\rangle\right]\right. \\
& \left.+\frac{3}{8} \frac{C_{3}}{C_{1}+\frac{1}{3} C_{2}}\left(m_{\mathrm{e}} c^{2}\right)^{2}\left[\left\langle\epsilon_{v}\right\rangle-\frac{\left\langle\epsilon_{\mathrm{e}}^{2}\right\rangle}{\left\langle\epsilon_{\mathrm{e}}\right\rangle}\right]\right\},
\end{aligned}
$$

where e can be $\mathrm{e}^{+}$or $\mathrm{e}^{-}$and $v$ stands for neutrinos or antineutrinos of all flavours and the constants $C_{1}, C_{2}, C_{3}$ for the different combinations are listed in Table D.1. The term $3 m_{\mathrm{e}} c^{2} / 4$ in the bracket results from a merge of the rate expressions for the limits of relativistic and non-relativistic electrons. In the latter case the neutrino-electron scattering cross section is proportional to $\epsilon_{v} /\left(m_{\mathrm{e}} c^{2}\right)$ for $\epsilon_{v} \gg m_{\mathrm{e}} c^{2}$ (cf. Sehgal 1974).

Every transfer by neutrino-nucleon scattering, which is only "nearly conservative", is taken into account following Tubbs (1979). The corresponding rate is (see Janka 1991b):

$$
\begin{aligned}
Q_{v \mathrm{~N}}= & \frac{1}{4} \frac{\sigma_{0} c}{\left(m_{\mathrm{e}} c^{2}\right)^{2}} C_{\mathrm{N}} \mathcal{E}_{\mathrm{N}} \frac{n_{\mathrm{N}}}{m_{\mathrm{N}} c^{2}}\left\{\left\langle\epsilon_{v}^{4}\right\rangle-6 T\left\langle\epsilon_{v}^{3}\right\rangle\right\} \\
& \times \frac{L_{e, v}}{4 \pi r^{2} c f_{v}\left\langle\epsilon_{v}\right\rangle}
\end{aligned}
$$

with

$C_{\mathrm{N}} \mathcal{E}_{\mathrm{N}}=\left\{\begin{array}{lll}\frac{2}{3}\left[\left(C_{\mathrm{V}}-1\right)^{2}+\frac{5}{4} \alpha^{2}\right] & \text { for } & \mathrm{N}=\mathrm{p}, \\ \frac{1}{6}\left(1+5 \alpha^{2}\right) & \text { for } & \mathrm{N}=\mathrm{n} .\end{array}\right.$

The symbol $v$ stands again for neutrinos and antineutrinos of all flavours. Also scattering contributions are included in the energy generation rate $Q^{+}$and absorption coefficient $\tilde{\kappa}$ used in Eq. (D.7). Considering scattering as an absorption process followed immediately by an emission process, we add the net energy exchange rates $Q_{v \mathrm{e}^{-}}, Q_{v \mathrm{e}^{+}}, Q_{v \mathrm{p}}$ and $Q_{v \mathrm{n}}$ to $Q_{v}^{-}$(used for computing $\tilde{\kappa}_{v}$ in Eq. (D.7)) when the rates are positive (i.e. in case of energy transfer from neutrinos to the stellar gas), and the absolute values of $Q_{v^{-}}, Q_{v \mathrm{e}^{+}}, Q_{v \mathrm{p}}$ and $Q_{v \mathrm{n}}$ to $Q_{v}^{+}$otherwise. The total neutrino energy source term to be used in the gas energy equation including the contributions from $v_{\mathrm{e}}$ and $\bar{v}_{\mathrm{e}}$ absorption and emission, $v \bar{v}$ 
pair production, and all scattering reactions is

$$
\begin{aligned}
Q_{\mathrm{E}}= & \sum_{v \in\left\{v_{\mathrm{e}}, \bar{\nu}_{\mathrm{e}}\right\}}\left(Q_{v}^{\mathrm{a}}-Q_{v}^{\mathrm{e}}\right)+\sum_{v \in\left\{v_{\mathrm{e}}, \nu_{\mu}, v_{\tau}\right\}}\left(Q_{v \bar{v}}^{\mathrm{ann}}-Q_{v \bar{v}}^{\mathrm{prod}}\right) \\
& +\sum_{v \in\left\{v_{\mathrm{e}}, v_{\mu}, v_{\tau}, \bar{\nu}_{\mathrm{e}}, \bar{\nu}_{\mu}, \bar{v}_{\tau}\right\}}\left(Q_{v \mathrm{e}^{+}}^{\mathrm{s}}+Q_{v \mathrm{e}^{-}}^{\mathrm{s}}+Q_{v \mathrm{p}}^{\mathrm{s}}+Q_{v \mathrm{n}}^{\mathrm{s}}\right),
\end{aligned}
$$

where $Q_{\bar{v}}^{\mathrm{ann}}=Q_{v}^{\mathrm{ann}}+Q_{\bar{v}}^{\mathrm{ann}}$ and $Q_{v \bar{v}}^{\mathrm{prod}}=Q_{v}^{\mathrm{prod}}+Q_{\bar{v}}^{\text {prod }}$.

In practise, however, the lepton number source term $Q_{\mathrm{N}}$ as well as the energy source term for the hydrodynamics part of the code is not computed from Eqs. (D.60) and (D.69), respectively, but from the luminosity change between points $\left(r^{i}, t^{n}\right)$ and $\left(r^{\star}, t^{\star}\right)$ (cf. Fig. D.1). The source terms $\tilde{Q}_{\mathrm{N}}^{i}$ and $\tilde{Q}_{\mathrm{E}}^{i}$ for a grid cell $i$ at time level $t^{n}$ are then given by

$\tilde{Q}_{\mathrm{N}}^{i}=\frac{L_{n}^{\mathrm{diff}}\left(r^{i}, t^{n}\right)-L_{n}^{\mathrm{diff}}\left(r^{\star}, t^{\star}\right)}{\Delta V_{i}}$,

$\tilde{Q}_{\mathrm{E}}^{i}=\frac{L_{e}^{\mathrm{tot}}\left(r^{i}, t^{n}\right)-L_{e}^{\mathrm{tot}}\left(r^{\star}, t^{\star}\right)}{\Delta V_{i}}$

where $\Delta V_{i}=\frac{4 \pi}{3}\left(r_{i}^{3}-r^{\star}\right)$ is the part of the cell volume crossed by the characteristic line between $\left(r^{i}, t^{n}\right)$ and $\left(r^{\star}, t^{\star}\right), L_{e}^{\text {tot }}$ is the sum of the luminosities of neutrinos and antineutrinos of all flavours, and $L^{\text {diff }}$ is the difference between the $v_{\mathrm{e}}$ and $\bar{v}_{\mathrm{e}}$ number fluxes, $L_{v_{\mathrm{e}}, n}-L_{\bar{v}_{\mathrm{e}}, n}$. Equations (D.70) and (D.71) work well as a description of the neutrino sources in the gas equations only, if the neutrino fluxes do not exhibit a large degree of variability on the radial and temporal scales of the $r-t$ cells. This, however, is reasonably well fulfilled in the context considered in this paper.

Finally, the outgoing neutrino fluxes transfer also momentum to the stellar fluid. To account for this, we include a momentum source term $Q_{\mathrm{M}}$ which enters the Euler equation of the hydrodynamics solver. It is sufficient to include only the most important reactions, by which neutrinos transfer momentum, i.e. $v_{\mathrm{e}}$ and $\bar{v}_{\mathrm{e}}$ absorption on $\mathrm{n}$ and $\mathrm{p}$, respectively, and the scattering processes of $v$ and $\bar{v}$ of all flavours off nucleons and nuclei (pair processes and electron/positron scattering can be safely ignored). For a neutrino or antineutrino $v$, the corresponding rate (in units of $\mathrm{erg} / \mathrm{cm}^{4}$ ) is

$Q_{\mathrm{M}}^{v}=\frac{L_{e, v}}{4 \pi r^{2} c}\left(\frac{\left\langle\kappa_{v}^{\mathrm{a}} \epsilon_{v}\right\rangle}{\left\langle\epsilon_{v}\right\rangle}+\sum_{i \in\left\{\mathrm{p}, \mathrm{n}, A_{j}\right\}} \frac{\left\langle\kappa_{\mathrm{t},}^{\mathrm{s}, i} \epsilon_{v}\right\rangle}{\left\langle\epsilon_{v}\right\rangle}\right)$,

where the first term in the sum is relevant only for $v_{\mathrm{e}}$ and $\bar{v}_{\mathrm{e}}$. The energy averages of the scattering transport opacities, $\kappa_{\mathrm{t}, v}^{\mathrm{s}, i}$, and of the absorption opacities, $\kappa_{v}^{\mathrm{a}}$, all weighted by the neutrino energy, are given by

$$
\begin{aligned}
\left\langle\kappa_{\mathrm{t}, v}^{\mathrm{s}, \mathrm{p}} \epsilon_{v}\right\rangle= & \frac{1}{6}\left[\frac{5}{4} \alpha^{2}+\left(C_{\mathrm{V}}-1\right)^{2}\right] \frac{\sigma_{0}}{\left(m_{\mathrm{e}} c^{2}\right)^{2}}\left\langle\epsilon_{v}^{3}\right\rangle n_{\mathrm{p}}, \\
\left\langle\kappa_{\mathrm{t}, v}^{\mathrm{s}, \mathrm{n}} \epsilon_{v}\right\rangle= & \frac{5 \alpha^{2}+1}{24} \frac{\sigma_{0}}{\left(m_{\mathrm{e}} c^{2}\right)^{2}}\left\langle\epsilon_{v}^{3}\right\rangle n_{\mathrm{n}}, \\
\left\langle\kappa_{\mathrm{t}, v}^{\mathrm{s}, A_{j}} \epsilon_{v}\right\rangle= & \frac{1}{6} A_{j}^{2}\left[C_{\mathrm{A}}-1+\frac{Z_{j}}{A_{j}}\left(2-C_{\mathrm{A}}-C_{\mathrm{V}}\right)\right] \\
& \times \frac{\sigma_{0}}{\left(m_{\mathrm{e}} c^{2}\right)^{2}}\left\langle\epsilon_{v}^{3}\right\rangle n_{A_{j}}, \\
\left\langle\kappa_{v_{\mathrm{e}}}^{\mathrm{a}} \epsilon_{v_{\mathrm{e}}}\right\rangle= & \frac{1}{4}\left(3 \alpha^{2}+1\right) \frac{\sigma_{0}}{\left(m_{\mathrm{e}} c^{2}\right)^{2}} \\
& \times\left(\left\langle\epsilon_{v_{\mathrm{e}}^{3}}^{3}\right\rangle+2 \Delta\left\langle\epsilon_{v_{\mathrm{e}}}^{2}\right\rangle+\Delta^{2}\left\langle\epsilon_{v_{\mathrm{e}}}\right\rangle\right) \Theta\left(\left\langle\epsilon_{v_{\mathrm{e}}}\right\rangle\right),
\end{aligned}
$$

$$
\begin{aligned}
\left\langle\kappa_{\overline{\mathrm{v}}_{\mathrm{e}}}^{\mathrm{a}} \epsilon_{\bar{\nu}_{\mathrm{e}}}\right\rangle= & \frac{1}{4}\left(3 \alpha^{2}+1\right) \frac{\sigma_{0}}{\left(m_{\mathrm{e}} c^{2}\right)^{2}} \\
& \times\left(\left\langle\epsilon_{\bar{\nu}_{\mathrm{e}}}^{3}\right\rangle^{\star}+3 \Delta\left\langle\epsilon_{\bar{\nu}_{\mathrm{e}}}^{2}\right\rangle^{\star}+3 \Delta^{2}\left\langle\epsilon_{\bar{\nu}_{\mathrm{e}}}\right\rangle^{\star}+\Delta^{3}\left\langle\epsilon_{\overline{\mathrm{v}}_{\mathrm{e}}}^{0}\right\rangle^{\star}\right) .
\end{aligned}
$$

The energy moments $\left\langle\epsilon_{v}^{n}\right\rangle$ and $\left\langle\epsilon_{v}^{n}\right\rangle^{\star}$ are given in Eqs. (D.42) and (D.43). They are calculated using the nonequilibrium neutrino spectral parameters $T_{v}$ and $\eta_{v}$. The momentum source term in the equation of gas motion then reads

$$
Q_{\mathrm{M}}=\sum_{v \in\left\{v_{\mathrm{e}}, v_{\mu}, v_{\tau}, \bar{v}_{\mathrm{e}}, \bar{v}_{\mu}, \bar{v}_{\tau}\right\}} Q_{\mathrm{M}}^{v}
$$

It was not included in the simulations presented in this paper, but will be taken into account in future calculations.

The implementation of the source terms $\tilde{Q}_{\mathrm{N}}, \tilde{Q}_{\mathrm{E}}$, and $Q_{\mathrm{M}}$ into the framework of our PPM hydrodynamics code was discussed in detail by Rampp \& Janka (2002) and Buras et al. (2006a).

We finish by pointing out that the approximative neutrino transport scheme developed here employs two basic assumptions, which are radical simplifications of the true situation:

1. In deriving Eq. (D.7) from the transport equation we assumed that the flux factor $f(r, t)$ is a known function, although it is actually dependent on the solution of the transport problem (see Eq. (D.4)). Equation (D.7) certainly has the advantage of analytic simplicity, but also has a severe disadvantage: The source terms can be very large and the numerical use requires a very fine grid zoning at high optical depths. The cell size should fulfill the constraint that the optical depth of the cell stays around unity or less. Moreover, the implementation of the source terms in (D.7) and the medium sources (Eqs. (D.70), (D.71)) is not symmetric and the numerical scheme does not strictly conserve the total lepton number and total energy of neutrinos plus gas.

2. For treating the spectral dependence, we made the assumption that the neutrino phase space distribution function can be factorised into a product of an angle-dependent function $g_{v}$ and an energy-dependent term, which we assume to be of Fermi-Dirac shape. This certainly constrains the spectral shape, but the factorisation also implies that the fluxfactor is assumed not to be an energy-dependent quantity. This in turn means that the mean energy of the neutrinos flux, $\left\langle\epsilon_{v}\right\rangle_{\text {flux }} \equiv L_{e, v}(r, t) / L_{n, v}(r, t)$ is identical with the mean energy of the local neutrino density, $\left\langle\epsilon_{v}\right\rangle_{\text {local }} \equiv E_{v}(r, t) / n_{v}(r, t)$. This is certainly a problematic simplification in view of the fact that the neutrino interactions with the stellar medium are strongly energy-dependent.

Nevertheless, the described neutrino transport treatment represents a practical approximation which is able to reproduce basic features of more detailed transport solutions and yields agreement with those even beyond the purely qualitative level. 